\title{
A Family of Calabi-Yau Varieties and Potential Automorphy II
}

\author{
To Mikio Sato with admiration
}

by

Tom Barnet-Lamb, David Geraghty, Michael Harris and Richard Taylor

\begin{abstract}
We prove new potential modularity theorems for $n$-dimensional essentially self-dual $l$ adic representations of the absolute Galois group of a totally real field. Most notably, in the ordinary case we prove quite a general result. Our results suffice to show that all the symmetric powers of any non-CM, holomorphic, cuspidal, elliptic modular newform of weight greater than one are potentially cuspidal automorphic. This in turns proves the Sato-Tate conjecture for such forms. (In passing we also note that the Sato-Tate conjecture can now be proved for any elliptic curve over a totally real field.)
\end{abstract}

2010 Mathematics Subject Classification: 11F80, 11F11, 11R45.

Keywords: automorphic representation, Galois representation, Dwork family, Sato-Tate conjecture.

\section{Introduction}

In this paper we prove potential modularity theorems for $n$-dimensional essentially self-dual $l$-adic representations of the absolute Galois group of a totally real field which extend those of [HSBT]. In the ordinary case we prove quite a general result. We also prove a result in the 'niveau two' case, but one with much more restrictive

This is a contribution to the special issue "The golden jubilee of algebraic analysis".

Communicated by M. Kashiwara. Received July 6, 2009. Revised April 2, 2010.

T. Barnet-Lamb: Department of Mathematics, Brandeis University,

Waltham, MA 02454, U.S.A.;

e-mail: tbl@brandeis.edu

D. Geraghty: School of Mathematics, Institute for Advanced Study, Princeton, NJ 08540, U.S.A.; e-mail: geraghty@math.ias.edu

M. Harris: Institut de Mathématiques de Jussieu, U.M.R. 7586 du CNRS,

75252 Paris Cedex 05, France;

e-mail: harris@math.jussieu.fr

R. Taylor: Department of Mathematics, Harvard University, Cambridge, MA 02138, U.S.A.;

e-mail: rtaylor@math.harvard.edu

(C) 2011 Research Institute for Mathematical Sciences, Kyoto University. All rights reserved. 
hypotheses. The combination of these two results does however suffice to show that all the symmetric powers of any non-CM, regular algebraic (i.e. holomorphic weight $\geq 2$ in the classical language) cuspidal automorphic representation of $G L_{2}\left(\mathbb{A}_{\mathbb{Q}}\right)$ (cuspidal newform in the classical language) are potentially cuspidal automorphic. This in turns proves the Sato-Tate conjecture for any non-CM, regular algebraic, cuspidal automorphic representation of $G L_{2}\left(\mathbb{A}_{\mathbb{Q}}\right)$. (In passing we also remark that the Sato-Tate conjecture can also now be proved for any elliptic curve over a totally real field.)

We will now state some of our theorems more precisely.

Theorem A. Suppose that $F$ is a totally real field, that $n$ is a positive integer, that $l>2 n$ is a rational prime, and that

$$
r: \operatorname{Gal}(\bar{F} / F) \rightarrow G L_{n}\left(\mathcal{O}_{\overline{\mathbb{Q}}_{l}}\right)
$$

is a continuous representation which is unramified at all but finitely many primes. Suppose also that there is a perfect pairing $\langle$,$\rangle on \overline{\mathbb{Q}}_{l}^{n}$ and a character $\mu$ : $\operatorname{Gal}(\bar{F} / F) \rightarrow \mathcal{O}_{\overline{\mathbb{Q}}_{l}}^{\times}$such that

$$
\langle g x, g y\rangle=\mu(g)\langle x, y\rangle
$$

for all $x, y \in \overline{\mathbb{Q}}_{l}^{n}$ and all $g \in \operatorname{Gal}(\bar{F} / F)$. Let $\bar{r}$ denote the semisimplification of the reduction of $r$ modulo the maximal ideal of $\mathcal{O}_{\overline{\mathbb{Q}}_{l}}$. Suppose moreover that $r$ enjoys the following properties:

1. [odd] There is an $\varepsilon \in\{ \pm 1\}$ such that

- for every place $v \mid \infty$ of $F$ we have $\mu\left(c_{v}\right)=\varepsilon$ (where $c_{v}$ denotes complex conjugation at $v)$;

- for every $x, y \in \overline{\mathbb{Q}}_{l}^{n}$ we have $\langle y, x\rangle=\varepsilon\langle x, y\rangle$.

2. [regular and ordinary] For every place $v \mid l$ of $F$ there is a $\operatorname{Gal}\left(\bar{F}_{v} / F_{v}\right)$-invariant decreasing filtration $\mathrm{Fil}_{v}^{i}$ on $\overline{\mathbb{Q}}_{l}^{n}$ such that for $i=1, \ldots, n$ the graded piece $\operatorname{gr}_{v}^{i} \overline{\mathbb{Q}}_{l}^{n}$ is one-dimensional and $\operatorname{Gal}\left(\bar{F}_{v} / F_{v}\right)$ acts on it by a character $\chi_{v, i}$. Moreover for $v \mid l$ and $i=1, \ldots, n$ there is an open subgroup of $F_{v}^{\times}$on which we have

$$
\chi_{v, i}\left(\operatorname{Art}_{F_{v}} \alpha\right)=\prod_{\tau: F_{v} \hookrightarrow \overline{\mathbb{Q}}_{l}}(\tau \alpha)^{b_{\tau, i}}
$$

for some rational integers $b_{\tau, 1}<b_{\tau, 2}<\cdots<b_{\tau, n}$.

3. $\left[\bar{F}^{\operatorname{kerad} \bar{r}}\left(\zeta_{l}\right): \bar{F}^{\text {ker ad } \bar{r}}\right]>2$.

4. $H^{0}\left(\bar{r}\left(\operatorname{Gal}\left(\bar{F} / F\left(\zeta_{l}\right)\right)\right)\right.$, ad $\left.\bar{r}\right)=\overline{\mathbb{F}}_{l} 1_{n}$ and $H^{1}\left(\bar{r}\left(\operatorname{Gal}\left(\bar{F} / F\left(\zeta_{l}\right)\right)\right)\right.$, ad $\left.\bar{r}\right)=(0)$. 
5. For all irreducible $\overline{\mathbb{F}}_{l}[\operatorname{Gal}(\bar{F} / F)]$-submodules $W$ of ad $\bar{r}$ we can find $\sigma \in$ $\operatorname{Gal}(\bar{F} / F)$ and $\alpha \in \overline{\mathbb{F}}_{l}$ with the following properties:

- the element $\alpha$ is a simple root of the characteristic polynomial of $\bar{r}(\sigma)$ and if $\beta$ is another root then $\alpha^{2} \neq \beta^{2}$;

- $\pi_{\bar{r}(\sigma), \alpha} \circ W \circ i_{\bar{r}(\sigma), \alpha} \neq(0)$ (where $i_{\bar{r}(\sigma), \alpha}$ (resp. $\pi_{\bar{r}(\sigma), \alpha}$ ) denotes the inclusion of (resp. $\bar{r}(\sigma)$-equivariant projection to) the $\alpha$-eigenspace of $\bar{r}(\sigma)$ ).

Then there is a Galois totally real extension $F^{\prime} / F$ such that $\left.r\right|_{\operatorname{Gal}\left(\bar{F} / F^{\prime}\right)}$ is automorphic.

See Theorem 7.5. We remark that the fourth and fifth conditions will be satisfied if, for instance, the following three conditions hold: $l$ is sufficiently large compared to $n$ and $\left[\mathbb{F}: \mathbb{F}_{l}\right] ; \bar{r}(\operatorname{Gal}(\bar{F} / F))$ has no $l$-power quotient; and $\bar{r}(\operatorname{Gal}(\bar{F} / F)) \supset$ Symm $^{n-1} S L_{2}\left(\mathbb{F}_{l}\right)$. (See Lemma 7.4 )

Theorem B. Suppose that $f$ is a holomorphic, elliptic modular newform of weight $k \geq 2$, level $N$ and nebentypus character $\psi:(\mathbb{Z} / N \mathbb{Z})^{\times} \rightarrow \mathbb{C}^{\times}$. Write

$$
f(z)=e^{2 \pi i z}+\sum_{n=2}^{\infty} a_{n} e^{2 \pi i n z},
$$

and for $p \nmid N$ a prime write $\left\{\alpha_{p}, \beta_{p}\right\}$ for the roots of

$$
X^{2}-a_{p} X+\psi(p) p^{k-1}
$$

If $m \in \mathbb{Z}_{>0}$ and if $\chi:(\mathbb{Z} / M \mathbb{Z})^{\times} \rightarrow \mathbb{C}^{\times}$is a Dirichlet character we will write $L^{(N M)}\left(\left(\operatorname{Symm}^{m} f\right) \times \chi, s\right)$ for the partial L-function

$$
\prod_{p \nmid N M}\left(1-\alpha_{p}^{m} \chi(p) / p^{s}\right)^{-1}\left(1-\alpha_{p}^{m-1} \beta_{p} \chi(p) / p^{s}\right)^{-1} \ldots\left(1-\beta_{p}^{m} \chi(p) / p^{s}\right)^{-1}
$$

which converges absolutely (uniformly on compact sets) to a holomorphic function in $\operatorname{Re} s>m(k-1) / 2+1$. Also write ${ }^{c} f$ for the newform

$$
{ }^{c} f(z)=e^{2 \pi i z}+\sum_{n=2}^{\infty}{ }^{c} a_{n} e^{2 \pi i n z} .
$$

Suppose further that $f$ is not $C M$, i.e. there does not exist an imaginary quadratic field $M$ such that $a_{p}=0$ for all $p \nmid N$ which are inert in $M$.

1. If $m \in \mathbb{Z}_{>0}$ then there is a Galois totally real number field $F / \mathbb{Q}$ and a cuspidal automorphic representation $S_{F}^{m}(f)$ of $G L_{m+1}\left(\mathbb{A}_{F}\right)$ such that for all primes $v \mid p \nmid N$ of $F$, the local component $S_{F}^{m}(f)_{v}$ is unramified and has Satake parameters

$$
\left\{\alpha_{p}^{m\left[k(v): \mathbb{F}_{p}\right]}, \alpha_{p}^{(m-1)\left[k(v): \mathbb{F}_{p}\right]} \beta_{p}^{\left[k(v): \mathbb{F}_{p}\right]}, \ldots, \beta_{p}^{m\left[k(v): \mathbb{F}_{p}\right]}\right\}
$$


2. If $m \in \mathbb{Z}_{>0}$ and if $\chi:(\mathbb{Z} / M \mathbb{Z})^{\times} \rightarrow \mathbb{C}^{\times}$is a Dirichlet character then the $L$ function $L^{(N M)}\left(\left(\operatorname{Symm}^{m} f\right) \times \chi, s\right)$ has meromorphic continuation to the whole complex plane, and is holomorphic and non-zero in $\operatorname{Re} s \geq m(k-1) / 2+1$. Moreover the expected functional equation relating $L^{(N M)}\left(\left(\operatorname{Symm}^{m} f\right) \times \chi, s\right)$ to $L^{(N M)}\left(\left(\operatorname{Symm}^{m}\left({ }^{c} f\right)\right) \times \chi^{-1}, m(k-1)+1-s\right)$ holds.

3. If $\zeta$ is a root of unity with $\zeta^{2}$ in the image of $\psi$ then, as $p$ varies over primes with $\psi(p)=\zeta^{2}$, the numbers $a_{p} /\left(2 p^{(k-1) / 2} \zeta\right) \in \mathbb{R}$ are equidistributed in $[-1,1]$ with respect to the measure $(2 / \pi) \sqrt{1-t^{2}} d t$.

See Section 8 . This theorem was proved in HSBT in the special case that $k=2$ and there exists a prime $p$ which exactly divides $N$ but does not divide the conductor of $\psi$. In Gee this was extended to the case that $k=3$ and there exists a prime $p$ which exactly divides $N$ but does not divide the conductor of $\psi$.

As examples of Theorem $B$ we mention the following special cases, though many similar consequences are also available.

Corollary C. Write

$$
\sum_{n=1}^{\infty} \tau(n) q^{n}=q \prod_{n}\left(1-q^{n}\right)^{24},
$$

i.e. $\tau(n)$ denotes Ramanujan's $\tau$ function. Then the numbers $\tau(p) /\left(2 p^{11 / 2}\right)$ are equidistributed in $[-1,1]$ with respect to the measure $(2 / \pi) \sqrt{1-t^{2}} d t$.

Corollary D. Let $N_{12}(n)$ denote the number of elements of $\mathbb{Z}^{12}$ with Euclidean norm $\sqrt{n}$, i.e. the number of ways $n$ can be written as the sum of 12 perfect squares (where the order matters). As p runs over prime numbers,

$$
\left(N_{12}(p)-8\left(p^{5}+1\right)\right) /\left(32 p^{5 / 2}\right)
$$

lies in $[-1,1]$ and these numbers are equidistributed in $[-1,1]$ with respect to the measure $(2 / \pi) \sqrt{1-t^{2}} d t$.

There are four main advances which make these results possible, none original to this paper:

1. The construction of Galois representations for all regular algebraic, essentially conjugate self-dual, cuspidal automorphic representations of $G L_{n}$ of the adeles of a CM field. This was possible because of work of Laumon and Ngo [LN] and of Waldspurger (see for instance [W]). It was carried out on the one hand by Shin Sh and on the other by a group of mathematicians including Chenevier, Clozel, Labesse and M.H. working together (see [CHLN]). (This allows Guerberoff [Gu] to remove the 'discrete series at a finite place' hypothesis in [CHT] and Tay.) 
2. The generalization by D.G. (see Ger) of the modularity lifting theorems of CHT and Tay to the ordinary case. This generalization has two important advantages for applications to potential modularity theorems. Firstly it allows one to work with $l$-adic representations of the absolute Galois group of a totally real number field in which $l$ ramifies. Secondly it allows one to use Hida families to 'change weight'.

3. The analysis of the whole of the cohomology of members of the Dwork family of hypersurfaces that was begun in [Ka2] and carried further in GHK, BL1] and BL2]. This allows us to show that representations $\bar{r}: \operatorname{Gal}(\overline{\mathbb{Q}} / \mathbb{Q}) \rightarrow G S p_{n}\left(\overline{\mathbb{F}}_{l}\right)$ with multiplier $\epsilon_{l}^{1-n}$ are potentially automorphic.

4. The invention of the "tensoring with an "induced from character" representation' trick by M.H. in [H]. Tensoring by an 'induced from character' representation firstly allows us to turn any essentially self-dual representation $\bar{r}: \operatorname{Gal}(\overline{\mathbb{Q}} / \mathbb{Q}) \rightarrow G L_{n}\left(\overline{\mathbb{F}}_{l}\right)$ into one with a symplectic essential self-duality with multiplier $\epsilon_{l}^{1-n}$, to which one can apply our analysis of the Dwork family to obtain potential automorphy.

Perhaps more importantly, it also allows us to replace a representation $r: \operatorname{Gal}(\overline{\mathbb{Q}} / \mathbb{Q}) \rightarrow G L_{n}\left(\overline{\mathbb{Q}}_{l}\right)$ with equally spaced Hodge-Tate numbers by one which has Hodge-Tate numbers $\{0,1, \ldots, n-1\}$. In the case that $r$ is ordinary this step is not needed, because D.G.'s automorphy lifting theorem (see Ger] allows for a change of weight. However in the 'niveau two' case, we are forced to use the automorphy lifting theorem of $\mathrm{Gu}$, which does not allow for a change of weight. Because the constituents of the cohomology of the Dwork family have consecutive Hodge-Tate numbers, this forces us (in this 'niveau two' case) to work with an $r$ with Hodge-Tate numbers $\{0,1, \ldots, n-1\}$.

Given these innovations the writing of this paper was relatively routine, although there are a number of tricky, sometimes even subtle, points to take care of. We believe our method could also be made to work for Hilbert modular forms, but for simplicity we work only with elliptic modular forms in this paper. Shortly after this paper was written T.B.-L., Toby Gee and D.G. wrote a different proof both of Theorem B above and of the analogue for Hilbert modular forms [BLGG].

In the first section of this paper we recall the results of Sh and CHLN] on the construction of Galois representations for all regular algebraic, essentially conjugate self-dual, cuspidal automorphic representations of $G L_{n}$ of the adeles of a CM field. In the second section we recall the results of D.G. on modularity lifting theorems in the ordinary case as well as Guerberoff's $\mathrm{Gu}$ ] extension of the results of [CHT] and Tay. In the third section we take the opportunity to record some corrections to our earlier papers [CHT], Tay and [HSBT]. In the fourth and fifth 
sections we collect the information we will need about Dwork hypersurfaces. In Section 4 we use transcendental methods to prove Proposition 4.2. Our arguments follow those of GHK. In Section 5 we collect some more arithmetic information about Dwork hypersurfaces.

We are then finally in a position to prove some potential modularity theorems. In the sixth section we follow the method of [HSBT. We are able to prove theorems for symplectic representations with multiplier a specific power of the cyclotomic character. In the ordinary case we can do this in any (regular) weight and any coefficients (see Theorem 6.3). We also consider what one might call the 'niveau two case', but here we can only treat minimal weight and coefficients $\mathbb{Q}_{l}$ (see Theorem 6.4. There is even a slight restriction on which primes $l$ we can treat. In Section 7 we use the trick of $[\underline{H}$ to improve these potential modularity results. In particular we can relax the condition that the self-duality is symplectic (and the concomitant condition that we had an even-dimensional Galois representation) and the condition on the multiplier character (see Theorems 7.5 and 7.6). In the 'niveau two case' we can also somewhat relax the condition on the weight (we can treat 'equally spaced weights'), but in this case we also have to pay a price: we end up with a much more serious restriction on $l$. Finally in the eighth section we apply these potential modularity theorems to study symmetric powers of twodimensional Galois representations and the Sato-Tate conjecture.

It is a pleasure to dedicate this paper to Mikio Sato, who first raised some of these questions.

\section{Notation}

Let us establish some notation that we will use throughout the paper.

We will let $c$ denote complex conjugation (or sometimes $c_{v}$ if we wish to make clear to which real place $v$ it is attached).

If $F$ is a CM field then $F^{+}$is its maximal totally real subfield, and if $R \subset F$ is a subring then we will write $R^{+}$for $F^{+} \cap R$.

If $v$ is a prime or a point of a scheme we will let $k(v)$ denote its residue field.

For any positive integer $N$ let $\zeta_{N}$ denote a primitive $N^{\text {th }}$ root of unity. We will let $\epsilon_{l}$ denote the $l$-adic cyclotomic character defined by

$$
\sigma \zeta_{l^{r}}=\zeta_{l^{r}}^{\epsilon_{l}(\sigma) \bmod l^{r}}
$$

for any $r \in \mathbb{Z}_{>0}$ and any $\sigma$ in the Galois group. If $M$ is a $\mathbb{Z}_{l}$-module with Galois action we will write $M(n)$ for the $n^{\text {th }}$ Tate twist of $M$, i.e. $M\left(\epsilon_{l}^{n}\right)$.

If $F$ is a $p$-adic field with valuation $v$ then $F^{\mathrm{nr}}$ will denote its maximal unramified extension and $\operatorname{Frob}_{v} \in \operatorname{Gal}\left(F^{\mathrm{nr}} / F\right)$ will denote geometric Frobenius. Moreover $\operatorname{Art}_{F}: F^{\times} \rightarrow \operatorname{Gal}(\bar{F} / F)^{\mathrm{ab}}$ will denote the Artin map (normalized to take 
uniformizers to geometric Frobenius elements). We will let $F^{0}$ denote the maximal subfield of $F$ which is unramified over $\mathbb{Q}_{p}$. Also let $B_{\mathrm{DR}}, B_{\text {cris }}$ and $B_{\text {st }}$ denote Fontaine's rings. Thus $B_{\mathrm{DR}}$ is a topological $\bar{F}$-algebra with a filtration and a semilinear action of $\operatorname{Gal}(\bar{F} / F)$ which preserves the filtration. Similarly $B_{\text {cris }}$ and $B_{\text {st }}$ are topological $\left(F^{0}\right)^{\mathrm{nr}}$-algebras with a continuous semilinear action of $\mathrm{Gal}(\bar{F} / F)$ and a commuting Frob $_{p}^{-1}$ semilinear endomorphism $\phi$. In the case of $B_{\text {st }}$ there is a second endomorphism $N$ which commutes with the action of $\operatorname{Gal}(\bar{F} / F)$ and satisfies $p \phi N=N \phi$.

Continue to suppose that $F$ is a $p$-adic field. By a Steinberg representation of $G L_{n}(F)$ we shall mean a representation $\operatorname{Sp}_{n}(\psi)$ (in the notation of Section I.3 of [HT]) where $\psi$ is an unramified character of $F^{\times}$. We will write $\operatorname{rec}_{F}$ for the local Langlands correspondence, a bijection from irreducible smooth representations of $G L_{n}(F)$ over $\mathbb{C}$ to $n$-dimensional Frobenius semisimple Weil-Deligne representations of the Weil group $W_{F}$ of $F$. (See the introduction and/or Section VII.2 of [HT].) If $\pi$ (resp. $\sigma$ ) is an irreducible smooth representation of $G L_{n}(F)$ (resp. $\left.G L_{m}(F)\right)$ then $\pi \boxplus \sigma$ is the irreducible admissible representation of $G L_{n+m}(F)$ with $\operatorname{rec}_{F}(\pi \boxplus \sigma)=\operatorname{rec}_{F}(\pi) \oplus \operatorname{rec}_{F}(\sigma)$. If $l \neq p$ is another prime and if $\imath: \overline{\mathbb{Q}}_{l} \stackrel{\sim}{\rightarrow} \mathbb{C}$ then we will write $r_{l}\left(\imath^{-1} \pi\right)^{\vee}(1-n)$ for the Frobenius semisimple continuous representation of $\operatorname{Gal}(\bar{F} / F)$ into $G L_{n}\left(\overline{\mathbb{Q}}_{l}\right)$ which is associated to the Weil-Deligne representation $\imath^{-1} \operatorname{rec}_{F}\left(\pi \otimes|\operatorname{det}|_{F}^{(1-n) / 2}\right)$. (See Section 3.1 of [CHT]. We apologise for this horrible notation. We are using it to maintain consistent normalizations with some of our previous papers.)

If $F=\mathbb{R}$ or $\mathbb{C}$ one can make a similar definition of $\boxplus$. We will also write $\operatorname{Art}_{F}: F^{\times} \rightarrow \operatorname{Gal}(\bar{F} / F)$ and denote by sgn the unique epimorphism $\mathbb{R}^{\times} \rightarrow\{ \pm 1\}$.

Let $\mathbb{Q}_{l^{2}} / \mathbb{Q}_{l}$ denote the unramified quadratic extension, let $\mathbb{Z}_{l^{2}}$ denote its ring of integers and let

$$
\omega_{2}: \operatorname{Gal}\left(\overline{\mathbb{Q}}_{l} / \mathbb{Q}_{l^{2}}\right) \rightarrow \overline{\mathbb{F}}_{l}^{\times}
$$

be the character defined by

$$
\omega_{2}(\sigma)=\left(\sigma \sqrt[l^{2}-1]{l}\right) / \sqrt[l^{2}-1]{l} \bmod l .
$$

Also let

$$
\epsilon_{l}^{(2)}: \operatorname{Gal}\left(\overline{\mathbb{Q}}_{l} / \mathbb{Q}_{l^{2}}\right) \rightarrow \mathbb{Q}_{l^{2}}^{\times}
$$

denote the continuous character such that $\left(\epsilon_{l}^{(2)} \circ \operatorname{Art}_{\mathbb{Q}_{l^{2}}}\right)(l)=1$ and

$$
\left(\epsilon_{l}^{(2)} \circ \operatorname{Art}_{\mathbb{Q}_{l^{2}}}\right)(u)=u
$$

for $u \in \mathbb{Z}_{l^{2}}^{\times}$Then

$$
\omega_{2}=\epsilon_{l}^{(2)} \bmod l .
$$


If $F$ is a number field then

$$
\operatorname{Art}_{F}=\prod_{v} \operatorname{Art}_{F_{v}}: \mathbb{A}_{F}^{\times} / \overline{F^{\times}\left(F_{\infty}^{\times}\right)^{0}} \stackrel{\sim}{\rightarrow} \operatorname{Gal}(\bar{F} / F)^{\mathrm{ab}}
$$

will denote the Artin map. If $\pi_{1}, \ldots, \pi_{r}$ are cuspidal automorphic representations of $G L_{n_{1}}\left(\mathbb{A}_{F}\right), \ldots, G L_{n_{r}}\left(\mathbb{A}_{F}\right)$ then we will denote by $\pi_{1} \boxplus \cdots \boxplus \pi_{r}$ the irreducible representation of $G L_{n_{1}+\cdots+n_{r}}\left(\mathbb{A}_{F}\right)$ (or strictly speaking irreducible $G L_{n_{1}+\cdots+n_{r}}\left(\mathbb{A}_{F}^{\infty}\right) \times$ (Lie $\left.G L_{n_{1}+\cdots+n_{r}}\left(F_{\infty}\right), U_{\infty}\right)$-module) with

$$
\left(\pi_{1} \boxplus \cdots \boxplus \pi_{r}\right)_{v} \cong \pi_{1, v} \boxplus \cdots \boxplus \pi_{r, v}
$$

for all places $v$ of $F$.

Suppose that $V$ is a finite-dimensional vector space over a field $k$; that $g$ is a $k$-linear automorphism of $V$; and that $\alpha \in k$ is an eigenvalue of $g$. We will write $V_{g, \alpha}$ for the $\alpha$-generalized eigenspace of $g$. We will also write $i_{g, \alpha}$ for the canonical inclusion $V_{g, \alpha} \hookrightarrow V$ and $\pi_{g, \alpha}$ for the $g$-equivariant projection $V \rightarrow V_{g, \alpha}$. Thus $\pi_{g, \alpha} \circ i_{g, \alpha}=1_{V_{g, \alpha}}$. If $h \in \operatorname{End}_{k}(V)$ then

$$
\pi_{g, \alpha} h i_{g, \alpha} \in \operatorname{End}_{k}\left(V_{g, \alpha}\right) \text {. }
$$

In the special case that $\alpha$ is a simple root of the characteristic polynomial of $g$ we may think of

$$
\pi_{g, \alpha} h i_{g, \alpha} \in k \text {. }
$$

We will let $\mathfrak{g l}_{n}$ denote the space of $n \times n$-matrices with the adjoint action of $G L_{n}$, and $\mathfrak{g l}_{n}^{0}$ the subspace of trace zero matrices.

\section{$\S 1$. Galois representations for automorphic representations}

In this section we recall recent improvements to our ability to attach Galois representations to automorphic representations. None of the results are original to this paper, but we state them here, partly for the reader's convenience and partly to establish notation.

Suppose that $F$ is a number field and

$$
\chi: \mathbb{A}_{F}^{\times} / F^{\times} \rightarrow \mathbb{C}^{\times}
$$

is a continuous character for which there exists $a \in \mathbb{Z}^{\operatorname{Hom}(F, \mathbb{C})}$ such that

$$
\left.\chi\right|_{\left(F_{\infty}^{\times}\right)^{0}}: x \mapsto \prod_{\tau \in \operatorname{Hom}(F, \mathbb{C})}(\tau x)^{a_{\tau}}
$$

(i.e. an algebraic grossencharacter). Suppose also that $\imath: \overline{\mathbb{Q}}_{l} \stackrel{\sim}{\rightarrow} \mathbb{C}$. Then we define

$$
r_{l, \imath}(\chi): \operatorname{Gal}(\bar{F} / F) \rightarrow \overline{\mathbb{Q}}_{l}^{\times}
$$


to be the continuous character such that

$$
\imath\left(\left(r_{l, \imath}(\chi) \circ \operatorname{Art}_{F}\right)(x) \prod_{\tau \in \operatorname{Hom}(F, \mathbb{C})}\left(\imath^{-1} \tau\right)\left(x_{l}\right)^{-a_{\tau}}\right)=\chi(x) \prod_{\tau \in \operatorname{Hom}(F, \mathbb{C})}(\tau x)^{-a_{\tau}} .
$$

Now let $F$ be a totally real field. By a $R A E S D C$ (regular, algebraic, essentially self-dual, cuspidal) automorphic representation $\pi$ of $G L_{n}\left(\mathbb{A}_{F}\right)$ we mean a cuspidal automorphic representation such that

- $\pi^{\vee} \cong \pi \otimes(\chi \circ$ det $)$ for some continuous character $\chi: \mathbb{A}_{F}^{\times} / F^{\times} \rightarrow \mathbb{C}^{\times}$with $\chi_{v}(-1)$ independent of $v \mid \infty$,

- $\pi_{\infty}$ has the same infinitesimal character as some irreducible algebraic representation of the restriction of scalars of $G L_{n}$ from $F$ to $\mathbb{Q}$.

Note that $\chi$ is necessarily algebraic.

Now let $F$ be an imaginary CM field. By a $R A E C S D C$ (regular, algebraic, essentially conjugate self-dual, cuspidal) automorphic representation $\pi$ of $G L_{n}\left(\mathbb{A}_{F}\right)$ we mean a cuspidal automorphic representation such that

- there is a continuous character $\chi: \mathbb{A}_{F^{+}}^{\times} /\left(F^{+}\right)^{\times} \rightarrow \mathbb{C}^{\times}$with $\chi_{v}(-1)$ independent of $v \mid \infty$ such that

$$
\pi^{\vee} \cong \pi^{c} \otimes\left(\chi \circ \mathbf{N}_{F / F+} \circ \operatorname{det}\right)
$$

- $\pi_{\infty}$ has the same infinitesimal character as some irreducible algebraic representation of the restriction of scalars of $G L_{n}$ from $F$ to $\mathbb{Q}$.

Again note that $\chi$ is necessarily algebraic.

If $F$ is $\mathrm{CM}$ or totally real we will write $\left(\mathbb{Z}^{n}\right)^{\operatorname{Hom}(F, \mathbb{C}),+}$ for the set of $a=$ $\left(a_{\tau, i}\right) \in\left(\mathbb{Z}^{n}\right)^{\operatorname{Hom}(F, \mathbb{C})}$ satisfying

$$
a_{\tau, 1} \geq \cdots \geq a_{\tau, n}
$$

If $F^{\prime} / F$ is a finite totally real extension we define $a_{F^{\prime}} \in\left(\mathbb{Z}^{n}\right)^{\operatorname{Hom}\left(F^{\prime}, \mathbb{C}\right),+}$ by

$$
\left(a_{F^{\prime}}\right)_{\tau, i}=a_{\tau \mid F}, i
$$

If $a \in\left(\mathbb{Z}^{n}\right)^{\operatorname{Hom}(F, \mathbb{C}),+}$, let $\Xi_{a}$ denote the irreducible algebraic representation of $G L_{n}^{\operatorname{Hom}(F, \mathbb{C})}$ which is the tensor product over $\tau$ of the irreducible representations of $G L_{n}$ with highest weights $a_{\tau}$. We will say that a RAESDC (resp. RAECSDC) automorphic representation $\pi$ of $G L_{n}\left(\mathbb{A}_{F}\right)$ has weight $a$ if $\pi_{\infty}$ has the same infinitesimal character as $\Xi_{a}^{\vee}$.

The next two results are improvements to one of the main theorems of $[\mathrm{HT}]$. Following the proof of the fundamental lemma for endoscopy of unitary groups [LN] it was fairly clear to experts how to attempt to establish this improvement. 
One of us (M.H.) has organized a group of mathematicians to inter alia write a complete proof of this theorem (see [CHLN]). Meanwhile Shin gave a complete proof in the case that $a$ is sufficiently regular (Theorem 7.5 of [Sh]). The general case is deduced from this by Chenevier and Harris $[\mathrm{CH}]$ (see also [C]).

Theorem 1.1. Let $\imath: \overline{\mathbb{Q}}_{l} \stackrel{\sim}{\rightarrow} \mathbb{C}$. Let $F$ be a totally real field. Let $a \in\left(\mathbb{Z}^{n}\right)^{\operatorname{Hom}(F, \mathbb{C}),+}$. Suppose that $\pi$ is a RAESDC automorphic representation of $G L_{n}\left(\mathbb{A}_{F}\right)$ of weight a. Specifically suppose that $\pi^{\vee} \cong \pi \chi$ where $\chi: \mathbb{A}_{F}^{\times} /(F)^{\times} \rightarrow \mathbb{C}^{\times}$with $\chi_{v}(-1)$ independent of $v \mid \infty$. Then there is a continuous semisimple representation

$$
r_{l, l}(\pi): \operatorname{Gal}(\bar{F} / F) \rightarrow G L_{n}\left(\overline{\mathbb{Q}}_{l}\right)
$$

with the following properties:

1. For every prime $v \nmid l$ of $F$ we have

$$
\left.r_{l, l}(\pi)\right|_{\mathrm{Gal}\left(\bar{F}_{v} / F_{v}\right)} ^{\mathrm{ss}}=r_{l}\left(2^{-1} \pi_{v}\right)^{\vee}(1-n)^{\mathrm{ss}} .
$$

2. $r_{l, l}(\pi)^{\vee}=r_{l, l}(\pi) \epsilon_{l}^{n-1} r_{l, l}(\chi)$.

3. If $v \mid l$ is a prime of $F$ then the restriction $\left.r_{l, l}(\pi)\right|_{\mathrm{Gal}\left(\bar{F}_{v} / F_{v}\right)}$ is potentially semistable. Moreover if $\pi_{v}$ is unramified, if $F_{v}^{0}$ denotes the maximal unramified subextension of $F_{v} / \mathbb{Q}_{l}$ and if $\tau: F_{v}^{0} \hookrightarrow \overline{\mathbb{Q}}_{l}$ then $\left.r_{l, l}(\pi)\right|_{\mathrm{Gal}\left(\bar{F}_{v} / F_{v}\right)}$ is crystalline and the characteristic polynomial of $\phi^{\left[F_{v}^{0}: \mathbb{Q}_{l}\right]}$ on

$$
\left(r_{l, \imath}(\pi) \otimes_{\tau, F_{v}^{0}} B_{\text {cris }}\right)^{\operatorname{Gal}\left(\bar{F}_{v} / F_{v}\right)}
$$

equals the characteristic polynomial of $\imath^{-1} \operatorname{rec}_{F_{v}}\left(\pi_{v} \otimes|\operatorname{det}|_{v}^{(1-n) / 2}\right)\left(\operatorname{Frob}_{v}\right)$.

4. If $v \mid l$ is a prime of $F$ and if $\tau: F \hookrightarrow \overline{\mathbb{Q}}_{l}$ lies above $v$ then

$$
\operatorname{dim}_{\overline{\mathbb{Q}}_{l}} \operatorname{gr}^{i}\left(r_{l, l}(\pi) \otimes_{\tau, F_{v}} B_{\mathrm{DR}}\right)^{\operatorname{Gal}\left(\bar{F}_{v} / F_{v}\right)}=0
$$

unless $i=a_{\imath \tau, j}+n-j$ for some $j=1, \ldots, n$ in which case

$$
\operatorname{dim}_{\overline{\mathbb{Q}}_{l}} \operatorname{gr}^{i}\left(r_{l, \imath}(\pi) \otimes_{\tau, F_{v}} B_{\mathrm{DR}}\right)^{\operatorname{Gal}\left(\bar{F}_{v} / F_{v}\right)}=1 .
$$

This follows from Theorem 3.2.5 of $[\mathrm{CH}]$ in exactly the same way that Proposition 4.3.1 of [CHT] was deduced from Proposition 4.2.1 of that paper.

Theorem 1.2. Let $\imath: \overline{\mathbb{Q}}_{l} \stackrel{\sim}{\rightarrow} \mathbb{C}$. Let $F$ be an imaginary $C M$ field. Let $a \in$ $\left(\mathbb{Z}^{n}\right)^{\operatorname{Hom}(F, \mathbb{C}),+}$. Suppose that $\pi$ is a RAECSDC automorphic representation of $G L_{n}\left(\mathbb{A}_{F}\right)$ of weight a. Specifically suppose that $\pi^{\vee} \cong \pi^{c}\left(\chi \circ \mathbf{N}_{F / F^{+}}\right)$where $\chi$ : $\mathbb{A}_{F^{+}}^{\times} /\left(F^{+}\right)^{\times} \rightarrow \mathbb{C}^{\times}$is a continuous character with $\chi_{v}(-1)$ independent of $v \mid \infty$. Then there is a continuous semisimple representation

$$
r_{l, l}(\pi): \operatorname{Gal}(\bar{F} / F) \rightarrow G L_{n}\left(\overline{\mathbb{Q}}_{l}\right)
$$


with the following properties:

1. For every prime $v \nmid l$ of $F$ we have

$$
\left.r_{l, \imath}(\pi)\right|_{\operatorname{Gal}\left(\bar{F}_{v} / F_{v}\right)} ^{\mathrm{ss}}=r_{l}\left(\imath^{-1} \pi_{v}\right)^{\vee}(1-n)^{\mathrm{ss}} .
$$

2. $r_{l, \imath}(\pi)^{\vee}=\left.r_{l, \imath}(\pi)^{c} \epsilon_{l}^{n-1} r_{l, \imath}(\chi)\right|_{\operatorname{Gal}(\bar{F} / F)}$.

3. If $v \mid l$ is a prime of $F$ then the restriction $\left.r_{l, \imath}(\pi)\right|_{\mathrm{Gal}\left(\bar{F}_{v} / F_{v}\right)}$ is potentially semistable. Moreover if $\pi_{v}$ is unramified, if $F_{v}^{0}$ denotes the maximal unramified subextension of $F_{v} / \mathbb{Q}_{l}$ and if $\tau: F_{v}^{0} \hookrightarrow \overline{\mathbb{Q}}_{l}$ then $\left.r_{l, i}(\pi)\right|_{\mathrm{Gal}\left(\bar{F}_{v} / F_{v}\right)}$ is crystalline and the characteristic polynomial of $\phi^{\left[F_{v}^{0}: \mathbb{Q}_{l}\right]}$ on

$$
\left(r_{l, l}(\pi) \otimes_{\tau, F_{v}^{0}} B_{\text {cris }}\right)^{\operatorname{Gal}\left(\bar{F}_{v} / F_{v}\right)}
$$

equals the characteristic polynomial of $\imath^{-1} \operatorname{rec}_{F_{v}}\left(\pi_{v} \otimes|\operatorname{det}|_{v}^{(1-n) / 2}\right)\left(\operatorname{Frob}_{v}\right)$.

4. If $v \mid l$ is a prime of $F$ and if $\tau: F \hookrightarrow \overline{\mathbb{Q}}_{l}$ lies above $v$ then

$$
\operatorname{dim}_{\overline{\mathbb{Q}}_{l}} \operatorname{gr}^{i}\left(r_{l, l}(\pi) \otimes_{\tau, F_{v}} B_{\mathrm{DR}}\right)^{\operatorname{Gal}\left(\bar{F}_{v} / F_{v}\right)}=0
$$

unless $i=a_{\imath \tau, j}+n-j$ for some $j=1, \ldots, n$ in which case

$$
\operatorname{dim}_{\overline{\mathbb{Q}}_{l}} \operatorname{gr}^{i}\left(r_{l, \imath}(\pi) \otimes_{\tau, F_{v}} B_{\mathrm{DR}}\right)^{\operatorname{Gal}\left(\bar{F}_{v} / F_{v}\right)}=1 .
$$

Proof. Note that $\chi$ is algebraic. By Lemma 4.1.4 of CHT we can find an algebraic character $\psi$ of $\mathbb{A}_{F}^{\times} / F^{\times}$such that

$$
\chi \circ \mathbf{N}_{F / F^{+}}=\psi \psi^{c} .
$$

Then

$$
(\pi \psi)^{c} \cong(\pi \psi)^{\vee} .
$$

Theorem 3.2.5 of $\mathrm{CH}$ tells us that one can associate a Galois representation $r_{l, \imath}(\pi \psi)$ to $\pi \psi$ as in the theorem. Finally set

$$
r_{l, \imath}(\pi)=r_{l, \imath}(\pi \psi) \otimes r_{l, \imath}(\psi)^{-1}
$$

In both cases the representation $r_{l, \imath}(\pi)$ can be taken to be valued in $G L_{n}(\mathcal{O})$ where $\mathcal{O}$ is the ring of integers of some finite extension of $\mathbb{Q}_{l}$. Thus we can reduce it modulo the maximal ideal of $\mathcal{O}$ and semisimplify to obtain a continuous semisimple representation

$$
\bar{r}_{l, \imath}(\pi): \operatorname{Gal}(\bar{F} / F) \rightarrow G L_{n}\left(\overline{\mathbb{F}}_{l}\right)
$$

which is independent of the choices made.

Write $\left(\mathbb{Z}^{n}\right)^{\operatorname{Hom}\left(F, \overline{\mathbb{Q}}_{l}\right),+}$ for the set of $a \in\left(\mathbb{Z}^{n}\right)^{\operatorname{Hom}\left(F, \overline{\mathbb{Q}}_{l}\right)}$ satisfying

$$
a_{\tau, 1} \geq \cdots \geq a_{\tau, n}
$$


for all $\tau \in \operatorname{Hom}\left(F, \overline{\mathbb{Q}}_{l}\right)$. If $F^{\prime} / F$ is a finite totally real extension we define $a_{F^{\prime}} \in$ $\left(\mathbb{Z}^{n}\right)^{\operatorname{Hom}\left(F^{\prime}, \overline{\mathbb{Q}}_{l}\right),+}$ by

$$
\left(a_{F^{\prime}}\right)_{\tau, i}=a_{\left.\tau\right|_{F}, i} .
$$

If $\imath: \overline{\mathbb{Q}}_{l} \stackrel{\sim}{\rightarrow} \mathbb{C}$ and $a \in\left(\mathbb{Z}^{n}\right)^{\operatorname{Hom}\left(F, \overline{\mathbb{Q}}_{l}\right),+}$, then we define $\imath_{*} a \in\left(\mathbb{Z}^{n}\right)^{\operatorname{Hom}(F, \mathbb{C}),+}$ by

$$
\left(\imath_{*} a\right)_{\imath \tau, i}=a_{\tau, i} .
$$

Suppose that $F$ is either totally real or imaginary $\mathrm{CM}$ and that $a \in$ $\left(\mathbb{Z}^{n}\right)^{\operatorname{Hom}\left(F, \overline{\mathbb{Q}}_{l}\right),+}$ satisfies the conditions of the previous paragraph. We will call a continuous semisimple representation

$$
r: \operatorname{Gal}(\bar{F} / F) \rightarrow G L_{n}\left(\overline{\mathbb{Q}}_{l}\right) \quad\left(\text { resp. } \bar{r}: \operatorname{Gal}(\bar{F} / F) \rightarrow G L_{n}\left(\overline{\mathbb{F}}_{l}\right)\right)
$$

automorphic of weight $a$ if there is an isomorphism $\imath: \overline{\mathbb{Q}}_{l} \stackrel{\sim}{\rightarrow} \mathbb{C}$ and a RAESDC automorphic representation $\pi$ of $G L_{n}\left(\mathbb{A}_{F}\right)$ of weight $\imath_{*} a$ (resp. of weight $\imath_{*} a$ and with $\pi_{l}$ unramified) such that $r \cong r_{l, l}(\pi)$ (resp. $\bar{r} \cong \bar{r}_{l, l}(\pi)$ ). We will say that $r$ is automorphic of weight $a$ and level prime to $l$ if there is an isomorphism $\imath: \overline{\mathbb{Q}}_{l} \stackrel{\sim}{\rightarrow} \mathbb{C}$ and a RAESDC automorphic representation $\pi$ of $G L_{n}\left(\mathbb{A}_{F}\right)$ of weight $\imath_{*} a$ and with $\pi_{l}$ unramified such that $r \cong r_{l, l}(\pi)$.

The following standard lemmas extend and correct Lemmas 4.2.2, 4.3.2 and 4.3 .3 of [CHT], which were stated slightly incorrectly in [CHT]. (These lemmas allow one to check automorphy after first making a suitable soluble base extension and are used repeatedly in the sequel, as they were in [CHT, [ [HSBT] and Tay.)

Lemma 1.3. Suppose that $E / F$ is a soluble Galois extension of totally real fields; that

$$
\chi: \operatorname{Gal}(\bar{F} / F) \rightarrow \overline{\mathbb{Q}}_{l}^{\times}
$$

is a continuous character whose value at complex conjugations, $\chi\left(c_{v}\right)$, is independent of the infinite place $v$; and that

$$
r: \operatorname{Gal}(\bar{F} / F) \rightarrow G L_{n}\left(\overline{\mathbb{Q}}_{l}\right)
$$

is a continuous semisimple representation with

$$
r^{\vee} \cong r \otimes \chi
$$

Suppose also that $\left.r\right|_{\operatorname{Gal}(\bar{F} / E)}$ is irreducible and automorphic of weight a. Then:

1. $a_{\tau}=a_{\tau^{\prime}}$ if $\left.\tau\right|_{F}=\left.\tau^{\prime}\right|_{F}$ so we can define $a_{F}$ by $a_{F, \sigma}=a_{\widetilde{\sigma}}$ for any extension $\widetilde{\sigma}$ of $\sigma$ to $E$.

2. $r$ is automorphic over $F$ of weight $a_{F}$. 
Proof. Inductively we may reduce to the case that $E / F$ is cyclic of prime order. Suppose that $\operatorname{Gal}(E / F)=\langle\sigma\rangle$ and that $\left.r\right|_{\operatorname{Gal}(\bar{F} / E)}=r_{l, \imath}(\pi)$, for $\pi$ a RAESDC automorphic representation $\pi$ of $G L_{n}\left(\mathbb{A}_{E}\right)$ of weight $a$. Then $\left.\left.r\right|_{\operatorname{Gal}(\bar{F} / E)} ^{\sigma} \cong r\right|_{\operatorname{Gal}(\bar{F} / E)}$ so that $\pi^{\sigma} \cong \pi$. By Theorem 4.2 of [AC], $\pi$ descends to a cuspidal automorphic representation $\pi_{F}$ of $G L_{n}\left(\mathbb{A}_{F}\right)$. If $v \mid \infty$ is a place of $E$ then $\pi_{F,\left.v\right|_{F}} \cong \pi_{v}$, so that the first assertion of the lemma follows and $\pi_{F}$ has weight $a_{F}$.

Suppose that $\chi=r_{l, i}(\tilde{\chi})$. Then

$$
\pi^{\vee} \cong \pi \otimes\left(\left(\tilde{\chi}|\cdot|{ }_{F}^{1-n}\right) \circ \mathbf{N}_{E / F} \circ \operatorname{det}\right)
$$

It follows that

$$
\pi_{F}^{\vee} \cong \pi_{F} \otimes\left(\left(\tilde{\chi}|\cdot|{ }_{F}^{1-n} \mu\right) \circ \operatorname{det}\right)
$$

for some character $\mu$ of $\mathbb{A}_{F}^{\times} / F^{\times}\left(\mathbf{N}_{E / F} \mathbb{A}_{E}^{\times}\right)$. Thus $\pi_{F}$ is a RAESDC automorphic representation of $G L_{n}\left(\mathbb{A}_{F}\right)$. As $r$ and $r_{l, \imath}\left(\pi_{F}\right)$ are irreducible and have the same restriction to $\operatorname{Gal}(\bar{F} / E)$ we see that $r=r_{l, \imath}\left(\pi_{F}\right) \otimes \psi=r_{l, \imath}\left(\pi_{F} \otimes\left(\imath \circ \psi \circ \operatorname{Art}_{F}\right)\right)$ for some character $\psi$ of $\operatorname{Gal}(E / F)$. The lemma follows.

Lemma 1.4. Suppose that $E / F$ is a soluble Galois extension of imaginary $C M$ fields; that

$$
\chi: \operatorname{Gal}\left(\bar{F} / F^{+}\right) \rightarrow \overline{\mathbb{Q}}_{l}^{\times}
$$

is a continuous character with $\chi\left(c_{v}\right)$ independent of $v \mid \infty$; and that

$$
r: \operatorname{Gal}(\bar{F} / F) \rightarrow G L_{n}\left(\overline{\mathbb{Q}}_{l}\right)
$$

is a continuous semisimple representation with

$$
\left.r^{\vee} \cong r \otimes \chi\right|_{\operatorname{Gal}(\bar{F} / F)} .
$$

Suppose also that $\left.r\right|_{\operatorname{Gal}(\bar{F} / E)}$ is irreducible and automorphic of weight a. Then:

1. $a_{\tau}=a_{\tau^{\prime}}$ if $\left.\tau\right|_{F}=\left.\tau^{\prime}\right|_{F}$ so we can define $a_{F}$ by $a_{F, \sigma}=a_{\widetilde{\sigma}}$ for any extension $\widetilde{\sigma}$ of $\sigma$ to $E$.

2. $r$ is automorphic over $F$ of weight $a_{F}$.

Proof. Inductively we may reduce to the case that $E / F$ is cyclic of prime order. Suppose that $\operatorname{Gal}(E / F)=\langle\sigma\rangle$ and that $\left.r\right|_{\operatorname{Gal}(\bar{F} / E)}=r_{l, \imath}(\pi)$, for $\pi$ a RAECSDC automorphic representation $\pi$ of $G L_{n}\left(\mathbb{A}_{E}\right)$ of weight $a$. Then $\left.\left.r\right|_{\operatorname{Gal}(\bar{F} / E)} ^{\sigma} \cong r\right|_{\operatorname{Gal}(\bar{F} / E)}$ so that $\pi^{\sigma}=\pi$. By Theorem 4.2 of [AC], $\pi$ descends to a cuspidal automorphic representation $\pi_{F}$ of $G L_{n}\left(\mathbb{A}_{F}\right)$. If $v \mid \infty$ is a place of $E$ then $\pi_{F,\left.v\right|_{F}} \cong \pi_{v}$, so that the first assertion of the lemma follows and $\pi_{F}$ has weight $a_{F}$.

Suppose that $\chi=r_{l, i}(\tilde{\chi})$. Then

$$
\pi^{\vee} \cong \pi^{c} \otimes\left(\left(\tilde{\chi}|\cdot| F_{F^{+}}^{1-n}\right) \circ \mathbf{N}_{E / F^{+}} \circ \operatorname{det}\right) .
$$


It follows that

$$
\pi_{F}^{\vee} \cong \pi_{F}^{c} \otimes\left(\left(\widetilde{\chi}|\cdot| F^{+}{ }^{1-n} \mu\right) \circ \mathbf{N}_{F / F^{+}} \circ \operatorname{det}\right)
$$

for some character $\mu$ of

$$
\mathbb{A}_{F}^{\times} / F^{\times}\left(\mathbf{N}_{E / F} \mathbb{A}_{E}^{\times}\right) \stackrel{\sim}{\rightarrow} \mathbb{A}_{F^{+}}^{\times} /\left(F^{+}\right)^{\times}\left(\mathbf{N}_{E^{+} / F^{+}} \mathbb{A}_{E^{+}}^{\times}\right)
$$

(via the norm map $\mathbf{N}_{F / F^{+}}$). Thus $\pi_{F}$ is a RAECSDC automorphic representation of $G L_{n}\left(\mathbb{A}_{F}\right)$. As $r$ and $r_{l, l}\left(\pi_{F}\right)$ are irreducible and have the same restriction to $\operatorname{Gal}(\bar{F} / E)$ we see that $r=r_{l, \imath}\left(\pi_{F}\right) \otimes \psi=r_{l, \imath}\left(\pi_{F} \otimes\left(\imath \circ \psi \circ \operatorname{Art}_{F}\right)\right)$ for some character $\psi$ of $\operatorname{Gal}(E / F)$. The lemma follows.

Lemma 1.5. Suppose that $F$ is an imaginary $C M$ field; that

$$
\chi: \operatorname{Gal}\left(\bar{F} / F^{+}\right) \rightarrow \overline{\mathbb{Q}}_{l}^{\times}
$$

is a continuous character whose value at complex conjugations, $\chi\left(c_{v}\right)$, is independent of the infinite place $v$; and that

$$
r: \operatorname{Gal}\left(\bar{F} / F^{+}\right) \rightarrow G L_{n}\left(\overline{\mathbb{Q}}_{l}\right)
$$

is a continuous semisimple representation with

$$
r^{\vee} \cong r \otimes \chi
$$

Suppose also that $\left.r\right|_{\mathrm{Gal}(\bar{F} / F)}$ is irreducible and automorphic of weight a. Then:

1. $a_{\tau}=a_{\tau c}$ so we can define $a_{F^{+}}$by $a_{F^{+}, \sigma}=a_{\widetilde{\sigma}}$ for any extension $\tilde{\sigma}$ of $\sigma$ to $F$.

2. $r$ is automorphic over $F^{+}$of weight $a_{F^{+}}$.

Proof. Suppose that $\left.r\right|_{\operatorname{Gal}(\bar{F} / F)}=r_{l, l}(\pi)$, for $\pi$ a RAECSDC automorphic representation $\pi$ of $G L_{n}\left(\mathbb{A}_{F}\right)$ of weight $a$. As $\left.\left.r\right|_{\operatorname{Gal}(\bar{F} / F)} ^{c} \cong r\right|_{\operatorname{Gal}(\bar{F} / F)}$ we have $\pi^{c}=\pi$. By Theorem 4.2 of $\left[\mathrm{AC}\right.$ ], $\pi$ descends to a cuspidal automorphic representation $\pi_{F^{+}}$ of $G L_{n}\left(\mathbb{A}_{F^{+}}\right)$. If $v \mid \infty$ then $\pi_{v} \cong \pi_{v}^{c}$, so that the first assertion of the lemma follows and $\pi_{F^{+}}$has weight $a_{F^{+}}$.

Suppose that $\chi=r_{l, \imath}(\tilde{\chi})$. Then

$$
\pi^{\vee} \cong \pi^{c} \otimes\left(\left(\tilde{\chi}|\cdot|_{F^{+}}^{1-n}\right) \circ \mathbf{N}_{F / F^{+}} \circ \operatorname{det}\right) \text {. }
$$

It follows that

$$
\pi_{F^{+}}^{\vee} \cong \pi_{F^{+}} \otimes\left(\left(\tilde{\chi}|\cdot| F_{F^{+}}^{1-n} \mu\right) \circ \operatorname{det}\right)
$$

for some character $\mu$ of $\mathbb{A}_{F^{+}}^{\times} /\left(F^{+}\right)^{\times}\left(\mathbf{N}_{F / F^{+}} \mathbb{A}_{F}^{\times}\right)$. As $F$ is imaginary CM, $\mu_{v}(-1)$ is independent of $v \mid \infty$. Thus $\pi_{F^{+}}$is a RAESDC automorphic representation of $G L_{n}\left(\mathbb{A}_{F^{+}}\right)$. As $r$ and $r_{l, l}\left(\pi_{F^{+}}\right)$are irreducible and have the same restriction to 
$\operatorname{Gal}(\bar{F} / F)$ we see that $r=r_{l, \imath}\left(\pi_{F^{+}}\right) \otimes \psi=r_{l, \imath}\left(\pi_{F^{+}} \otimes\left(\imath \circ \psi \circ \operatorname{Art}_{F^{+}}\right)\right)$for some character $\psi$ of $\operatorname{Gal}\left(F / F^{+}\right)$. The lemma follows.

\section{$\S 2$. Automorphy lifting theorems}

In this section we recall two improvements to the modularity lifting theorems of [CHT] and Tay]. The first is due to Guerberoff [Gu and the second to David Geraghty Ger.

The next theorem is Theorem 5.2 of $[\mathrm{Gu}$.

Theorem 2.1 (Guerberoff). Let $F$ be a totally real field. Let $n \in \mathbb{Z}_{\geq 1}$ and let $l>n$ be a prime which is unramified in $F$. Let $r: \operatorname{Gal}(\bar{F} / F) \rightarrow G L_{n}\left(\overline{\mathbb{Q}}_{l}\right)$ be a continuous irreducible representation with the following properties. Let $\bar{r}$ denote the semisimplification of the reduction of $r$.

1. $r^{\vee} \cong r \epsilon_{l}^{n-1} \chi$ for some character $\chi: \operatorname{Gal}(\bar{F} / F) \rightarrow \overline{\mathbb{Q}}_{l}^{\times}$with $\chi\left(c_{v}\right)$ independent of $v \mid \infty$. (Here $c_{v}$ denotes a complex conjugation at $v$.)

2. $r$ ramifies at only finitely many primes.

3. For all places $v \mid l$ of $F,\left.r\right|_{\operatorname{Gal}\left(\bar{F}_{v} / F_{v}\right)}$ is crystalline.

4. There is an element $a \in\left(\mathbb{Z}^{n}\right)^{\operatorname{Hom}\left(F, \overline{\mathbb{Q}}_{l}\right),+}$ such that

- for all $\tau \in \operatorname{Hom}\left(F, \overline{\mathbb{Q}}_{l}\right)$ we have

$$
0 \leq a_{\tau, 1}+n-a_{\tau, n} \leq l-1
$$

- for all $\tau \in \operatorname{Hom}\left(F, \overline{\mathbb{Q}}_{l}\right)$ above a prime $v \mid l$ of $F$,

$$
\operatorname{dim}_{\overline{\mathbb{Q}}_{l}} \operatorname{gr}^{i}\left(r \otimes_{\tau, F_{v}} B_{\mathrm{DR}}\right)^{\operatorname{Gal}\left(\bar{F}_{v} / F_{v}\right)}=0
$$

unless $i=a_{\tau, j}+n-j$ for some $j=1, \ldots, n$ in which case

$$
\operatorname{dim}_{\overline{\mathbb{Q}}_{l}} \operatorname{gr}^{i}\left(r \otimes_{\tau, F_{v}} B_{\mathrm{DR}}\right)^{\operatorname{Gal}\left(\bar{F}_{v} / F_{v}\right)}=1 .
$$

5. $\zeta_{l} \notin(\bar{F})^{\operatorname{ker} \operatorname{ad} \bar{r}}$.

6. The image $\bar{r}\left(\operatorname{Gal}\left(\bar{F} / F\left(\zeta_{l}\right)\right)\right)$ is big (see Definition 2.5.1 of [CHT], which is the same as 1-big, defined in 7.2 below).

7. $\bar{r}$ is automorphic of weight a.

Then $r$ is automorphic of weight a and level prime to $l$.

If $a \in\left(\mathbb{Z}^{n}\right)^{\operatorname{Hom}\left(F, \overline{\mathbb{Q}}_{l}\right),+}$, we call a continuous representation

$$
r: \operatorname{Gal}(\bar{F} / F) \rightarrow G L_{n}\left(\overline{\mathbb{Q}}_{l}\right)
$$


ordinary of weight $a$ if for every prime $v \mid l$ of $F$ the following conditions are satisfied:

- there is a $\operatorname{Gal}\left(\bar{F}_{v} / F_{v}\right)$-invariant decreasing filtration $\mathrm{Fil}_{v}^{i}$ on $\overline{\mathbb{Q}}_{l}^{n}$ such that for $i=1, \ldots, n$ the graded piece $\operatorname{gr}_{v}^{i} \overline{\mathbb{Q}}_{l}^{n}$ is one-dimensional and $\operatorname{Gal}\left(\bar{F}_{v} / F_{v}\right)$ acts on it by a character $\chi_{v, i}$;

- there is an open subgroup of $F_{v}^{\times}$on which we have

$$
\chi_{v, i}\left(\operatorname{Art}_{F_{v}} \alpha\right)=\prod_{\tau: F_{v} \hookrightarrow \overline{\mathbb{Q}}_{l}}(\tau \alpha)^{i-n-a_{\tau, i}} .
$$

The first part of the following lemma is Lemma 2.7.6 of [Ger, the second part is proved in a similar way.

Lemma 2.2. 1. Suppose that $a \in\left(\mathbb{Z}^{n}\right)^{\operatorname{Hom}\left(F, \overline{\mathbb{Q}}_{l}\right),+}$ and that

$$
r: \operatorname{Gal}(\bar{F} / F) \rightarrow G L_{n}\left(\overline{\mathbb{Q}}_{l}\right)
$$

is crystalline at all primes $v \mid l$. We think of $v$ as a valuation $v: F_{v}^{\times} \rightarrow \mathbb{Z}$. If $\tau: F \hookrightarrow \overline{\mathbb{Q}}_{l}$ lies above $v$ suppose that

$$
\operatorname{dim}_{\overline{\mathbb{Q}}_{l}} \operatorname{gr}^{i}\left(r \otimes_{\tau, F_{v}} B_{\mathrm{DR}}\right)^{\operatorname{Gal}\left(\bar{F}_{v} / F_{v}\right)}=0
$$

unless $i=a_{\imath \tau, j}+n-j$ for some $j=1, \ldots, n$ in which case

$$
\operatorname{dim}_{\overline{\mathbb{Q}}_{l}} \operatorname{gr}^{i}\left(r \otimes_{\tau, F_{v}} B_{\mathrm{DR}}\right)^{\operatorname{Gal}\left(\bar{F}_{v} / F_{v}\right)}=1 .
$$

For $v \mid l$ let $\alpha_{v, 1}, \ldots, \alpha_{v, n}$ denote the roots of the characteristic polynomial of $\phi^{\left[F_{v}^{0}: \mathbb{Q}_{l}\right]}$ on

$$
\left(r \otimes_{\tau, F_{v}^{0}} B_{\text {cris }}\right)^{\operatorname{Gal}\left(\bar{F}_{v} / F_{v}\right)},
$$

for any $\tau: F_{v}^{0} \hookrightarrow \overline{\mathbb{Q}}_{l}$. (This characteristic polynomial is independent of the choice of $\tau$.) Let $\operatorname{val}_{v}$ denote the valuation on $\overline{\mathbb{Q}}_{l}$ normalized by $\operatorname{val}_{v}(l)=v(l)$.

(Thus $\operatorname{val}_{v} \circ \tau=v$ for any $\tau: F_{v} \hookrightarrow \bar{Q}_{l}$.) Arrange the $\alpha_{v, i}$ 's so that

$$
\operatorname{val}_{v}\left(\alpha_{v, 1}\right) \geq \operatorname{val}_{v}\left(\alpha_{v, 2}\right) \geq \cdots \geq \operatorname{val}_{v}\left(\alpha_{v, n}\right) .
$$

Then $r$ is ordinary of weight $a$ if and only if for all $v \mid l$ and all $i=1, \ldots, n$ we have

$$
\operatorname{val}_{v}\left(\alpha_{v, i}\right)=\sum_{\tau}\left(a_{\tau, i}+n-i\right)
$$

where $\tau$ runs over embeddings $F \hookrightarrow \overline{\mathbb{Q}}_{l}$ above $v$.

2. Suppose that $r: \operatorname{Gal}(\bar{F} / F) \rightarrow G L_{n}\left(\overline{\mathbb{Q}}_{l}\right)$ is semistable at all primes $v \mid l$. If $\tau: F \hookrightarrow \overline{\mathbb{Q}}_{l}$ lies above $v$ suppose that

$$
\operatorname{dim}_{\overline{\mathbb{Q}}_{l}} \operatorname{gr}^{i}\left(r \otimes_{\tau, F_{v}} B_{\mathrm{DR}}\right)^{\operatorname{Gal}\left(\bar{F}_{v} / F_{v}\right)}=0
$$


unless $i=0, \ldots, n-1$ in which case

$$
\operatorname{dim}_{\overline{\mathbb{Q}}_{l}} \operatorname{gr}^{i}\left(r \otimes_{\tau, F_{v}} B_{\mathrm{DR}}\right)^{\operatorname{Gal}\left(\bar{F}_{v} / F_{v}\right)}=1 .
$$

Suppose moreover that for some (and hence every) $\tau: F_{v}^{0} \hookrightarrow \overline{\mathbb{Q}}_{l}$ and for each $j=1, \ldots, n-1$ the kernel of $N^{j}$ on

$$
\left(r \otimes_{\tau, F_{v}^{0}} B_{\mathrm{st}}\right)^{\operatorname{Gal}\left(\bar{F}_{v} / F_{v}\right)}
$$

has $\overline{\mathbb{Q}}_{l}$-dimension $j$. Then $r$ is ordinary of weight 0 .

If $F$ is a totally real field we will call a continuous semisimple representation

$$
\bar{r}: \operatorname{Gal}(\bar{F} / F) \rightarrow G L_{n}\left(\overline{\mathbb{F}}_{l}\right)
$$

ordinarily automorphic of level prime to $l$ if there is $a \in\left(\mathbb{Z}^{n}\right)^{\operatorname{Hom}\left(F, \overline{\mathbb{Q}}_{l}\right),+}$, an isomorphism $\imath: \overline{\mathbb{Q}}_{l} \stackrel{\sim}{\rightarrow} \mathbb{C}$ and a RAESDC automorphic representation $\pi$ of $G L_{n}\left(\mathbb{A}_{F}\right)$ of weight $\imath_{*} a$, with $\pi_{l}$ unramified, such that $r_{l, \imath}(\pi)$ is ordinary of weight $a$ and $\bar{r} \cong \bar{r}_{l, \imath}(\pi)$. We will call $\bar{r}$ ordinarily Steinberg automorphic if there is an isomorphism $\imath: \overline{\mathbb{Q}}_{l} \stackrel{\sim}{\rightarrow} \mathbb{C}$ and a RAESDC automorphic representation $\pi$ of $G L_{n}\left(\mathbb{A}_{F}\right)$ of weight 0 , with $\pi_{v}$ a Steinberg representation for all $v \mid l$, such that $\bar{r} \cong \bar{r}_{l, l}(\pi)$.

The following is a slightly special case of Theorem 5.4.2 of [Ger].

Theorem 2.3 (Geraghty). Let $F$ be a totally real field. Let $n \in \mathbb{Z}_{\geq 1}$ and let $l>n$ be a prime. Let $r: \operatorname{Gal}(\bar{F} / F) \rightarrow G L_{n}\left(\overline{\mathbb{Q}}_{l}\right)$ be a continuous irreducible representation with the following properties. Let $\bar{r}$ denote the semisimplification of the reduction of $r$.

1. $r^{\vee} \cong r \epsilon_{l}^{n-1} \chi$ for some character $\chi: \operatorname{Gal}(\bar{F} / F) \rightarrow \overline{\mathbb{Q}}_{l}^{\times}$with $\chi\left(c_{v}\right)$ independent of $v \mid \infty$. (Here $c_{v}$ denotes a complex conjugation at $v$.)

2. $r$ ramifies at only finitely many primes.

3. $r$ is ordinary of weight a for some $a \in\left(\mathbb{Z}^{n}\right)^{\operatorname{Hom}\left(F, \overline{\mathbb{Q}}_{l}\right),+}$.

4. $(\bar{F})^{\operatorname{ker} a d} \bar{r}$ does not contain $F\left(\zeta_{l}\right)$.

5. The image $\bar{r}\left(\operatorname{Gal}\left(\bar{F} / F\left(\zeta_{l}\right)\right)\right)$ is big (see Definition 2.5.1 of [CHT]).

6. $\bar{r}$ is ordinarily automorphic of level prime to $l$ or ordinarily Steinberg automorphic.

Then $r$ is automorphic of weight a.

We remark that this theorem has two important advantages over earlier automorphy lifting theorems: it is not assumed that $l$ is unramified in $F$ and it is only assumed that $\bar{r}$ is ordinarily automorphic of some weight, not necessarily of weight $a$. 


\section{$\S 3$. Corrections to [CHT], [HSBT] and [Tay}

In this section we will digress to record some minor corrections to [CHT] and Tay.

\section{CHT}

The first assertion of Lemma 2.2.3 of [CHT is false. No proof was offered in CHT beyond 'the first assertion is clear'. We thank Shrenik Shah for asking a question that brought this to our attention. This part of Lemma 2.2.3 was not in fact used elsewhere in [CHT] or Tay, but for completeness will give a corrected version below.

Lemma 3.1. Suppose $\mathcal{O}$ is the ring of integers of a finite extension of $\mathbb{Q}_{l}$ with residue field $k$. Suppose $R$ is a reduced, noetherian, complete noetherian local $\mathcal{O}$ algebra with residue field $k$, but $R \neq k$. Suppose that $F \in R\left[\left[X_{1}, \ldots, X_{m}\right]\right]$ is a power series and that $F\left(a_{1}, \ldots, a_{m}\right)=0$ for all $a_{1}, \ldots, a_{m} \in \mathfrak{m}_{R}-\{0\}$. Then $F=0$.

Proof. By induction we may reduce to the case $m=1$. We may also reduce to the case that $R$ is a domain (as $R$ embeds in the product of $R / \mathfrak{p}$ as $\mathfrak{p}$ runs over non-maximal primes). Suppose $F(X) \neq 0$. Writing

$$
F(X)=\sum_{i=0}^{\infty} F_{i} X^{i}
$$

we may further suppose that $F_{0} \neq 0$ (if not, consider $F(X) / X^{r}$ for a suitable $r$ ). Then replacing $F$ by $F_{0}^{-1} F\left(F_{0} X\right)$ we may further suppose that $F_{0}=1$. In this case $F(a) \neq 0$ for all $a \in \mathfrak{m}_{R}$, and as long as $\mathfrak{m}_{R} \neq(0)$ we are done.

Now we state our corrected lemma. (In [CHT] the conditions in the third sentence of the lemma below were missed.)

Lemma 3.2. Keep the notation and assumptions of Section 2.2 of [CHT. Suppose that $\mathcal{I}$ is a $1_{n}+M_{n \times n}\left(\mathfrak{m}_{R_{q}^{\text {loc }}}\right)$-invariant ideal of $R_{q}^{\text {loc }}$. Assume also that $R_{q}^{\text {loc }} / \mathcal{I}$ is reduced and that $\mathcal{I} \neq \mathfrak{m}_{R_{q}^{\text {loc }}}$. Then the collection $\mathcal{D}_{\mathcal{I}}$ of all liftings $r$ of $\left.\bar{r}\right|_{\Delta_{q}}$ such that the kernel of the corresponding map $R_{q}^{\text {loc }} \rightarrow R$ contains $\mathcal{I}$ is a deformation problem.

Proof. The only non-trivial thing to check is that if $r: \Delta_{q} \rightarrow G L_{n}(R)$ is a lifting of $\left.\bar{r}\right|_{\Delta_{q}}$ in $\mathcal{D}_{\mathcal{I}}$ and if $g \in \operatorname{ker}\left(G L_{n}(R) \rightarrow G L_{n}(k)\right)$ then $\operatorname{grg}^{-1}$ also belongs to $\mathcal{D}_{\mathcal{I}}$. One immediately reduces to the (universal) special case that

$$
R=\left(R_{q}^{\mathrm{loc}} / \mathcal{I}\right)\left[\left[X_{i, j}\right]\right]_{i, j=1, \ldots, n},
$$


that $r$ is the push-forward of $r_{q}^{\text {univ }}$ and that $g=1_{n}+\left(X_{i, j}\right)$. Let

$$
\phi: R_{q}^{\mathrm{loc}} \rightarrow\left(R_{q}^{\mathrm{loc}} / \mathcal{I}\right)\left[\left[X_{i, j}\right]\right]
$$

be the morphism corresponding to

$$
\left(1_{n}+\left(X_{i, j}\right)\right) r_{q}^{\text {univ }}\left(1_{n}+\left(X_{i, j}\right)\right)^{-1}
$$

We must show that $\phi \mathcal{I}=(0)$. However for all $a_{i, j} \in \mathfrak{m}_{R_{q}^{\text {loc }}}$ we know that the map $R_{q}^{\text {loc }} \rightarrow R_{q}^{\text {loc }} / \mathcal{I}$ which is the composite of $\phi$ with evaluation $X_{i, j} \mapsto a_{i, j}$ sends $\mathcal{I}$ to $(0)$. The result now follows from the previous lemma.

We thank Bao Le Hung for pointing out that at the start of the proof of Lemma 3.2.2 we should remark that we can replace $M$ by its intersection with the $\bar{K}\left[G L_{n}\left(F_{w}\right)\right]$-submodule of $M \otimes_{\mathcal{O}} \bar{K}$ generated by $M^{\operatorname{Iw}(w)}$. After doing this, every irreducible subquotient of $M \otimes_{\mathcal{O}} \bar{K}$ has an $\operatorname{Iw}(w)$ fixed vector and so $M \otimes_{\mathcal{O}} \bar{K}$ has finite length over $\bar{K}\left[G L_{n}\left(F_{w}\right)\right]$. We may further assume that $R$ acts faithfully on $M$, which now implies that $R$ is finite over $\mathcal{O}$ and that $M \otimes \bar{K}$ is semisimple as a $\bar{K}\left[G L_{n}\left(F_{w}\right)\right]$-module. The rest of the proof then goes through unchanged.

The second item of the third list on page 95, the second item of the first list on page 11 and the second item of the third list on page 151 should all read 'For every place $v \mid \infty$...' rather than 'For a place $v \mid \infty \ldots$.'.

In the proof of Lemma 4.1.1 "any finite index subgroup of $\mathcal{O}_{F}^{\times}$" should read "any finite index subgroup of $\mathcal{O}_{F}[1 / S]^{\times}$".

As already remarked there are slight errors in the statements of Lemmas 4.2.2, 4.3.2 and 4.3.3 of [CHT]. Corrected versions, along with proofs, can be found in Lemmas 1.4 , 1.3 and 1.5 of this paper.

\section{Tay}

The second item of the second list on page 206 should read 'For every place $v \mid \infty \ldots$ ' rather than 'For a place $v \mid \infty \ldots$ '.

In addition D.G. pointed out that the assertions of the first paragraph of page 218 of Tay are not fully justified. The ring $R_{\left\{\chi_{v}\right\}, \infty}^{\square_{T}}$ is a completed tensor product of rings for which the corresponding assertions were proved in Section 2.4 of [CHT] and Proposition 3.1 of [Tay. What is missing is a justification that these properties are preserved under completed tensor products. R.T. has been able to locate few references for this sort of question, so we give here a proof of the required properties of completed tensor products.

Until the end of the proof of Lemma 3.3. $\mathcal{O}$ will denote a complete DVR with perfect residue field $k$, maximal ideal $\lambda$ and field of fractions $L$. We will let $\mathcal{C}_{\mathcal{O}}^{\text {noeth }}\left(\right.$ resp. $\left.\mathcal{C}_{k}^{\text {noeth }}\right)$ denote the category of complete noetherian local $\mathcal{O}$-algebras 
(resp. $k$-algebras) with residue field $k$. Note that if $A$ and $B$ are objects of $\mathcal{C}_{\mathcal{O}}^{\text {noeth }}$ (resp. $\mathcal{C}_{k}^{\text {noeth }}$ ) then so is $A \widehat{\otimes}_{\mathcal{O}} B$ (resp. $A \widehat{\otimes}_{k} B$ ). We will call an object $A$ of $\mathcal{C}_{\mathcal{O}}^{\text {noeth }}$ (resp. $\mathcal{C}_{k}^{\text {noeth }}$ ) geometrically integral if for all finite extensions $L^{\prime} / L$ (resp. $k^{\prime} / k$ ) the algebra $A \otimes_{\mathcal{O}} \mathcal{O}_{L^{\prime}}$ (resp. $A \otimes_{k} k^{\prime}$ ) is an integral domain. We will call an object $A$ of $\mathcal{C}_{\mathcal{O}}^{\text {noeth }}$ (resp. $\mathcal{C}_{k}^{\text {noeth }}$ ) geometrically irreducible if for all finite extensions $L^{\prime} / L$ (resp. $k^{\prime} / k$ ) the affine scheme $\operatorname{Spec} A \otimes_{\mathcal{O}} \mathcal{O}_{L^{\prime}}$ (resp. Spec $A \otimes_{k} k^{\prime}$ ) is irreducible.

Lemma 3.3. 1. If $A$ is an object of $\mathcal{C}_{\mathcal{O}}^{\text {noeth }}$ and $\mathfrak{p}$ is a maximal ideal of $A \otimes_{\mathcal{O}} L$ then the residue field $k(\mathfrak{p})$ is a finite extension of $L$ and the image of $A$ in $k(\mathfrak{p})$ is an order (i.e. an $\mathcal{O}$-subalgebra which is finitely generated as an $\mathcal{O}$-module which spans $k(\mathfrak{p})$ as an L-vector space). Moreover any prime ideal of $A \otimes_{\mathcal{O}} L$ is the intersection of the maximal ideals containing it.

2. If $A$ and $B$ are objects of $\mathcal{C}_{\mathcal{O}}^{\text {noeth }}$ which are flat over $\mathcal{O}$ and integral then the Krull dimension of $A \widehat{\otimes}_{\mathcal{O}} B$ is one less than the sum of the Krull dimensions of $A$ and of $B$.

3. If two objects $A$ and $B$ of $\mathcal{C}_{\mathcal{O}}^{\text {noeth }}$ are flat over $\mathcal{O}$ and geometrically integral (resp. irreducible) then $A \widehat{\otimes}_{\mathcal{O}} B$ is flat over $\mathcal{O}$ and geometrically integral (resp. irreducible).

4. If two objects $A$ and $B$ of $\mathcal{C}_{k}^{\text {noeth }}$ are geometrically integral (resp. irreducible) then $A \widehat{\otimes}_{\mathcal{O}} B$ is geometrically integral (resp. irreducible).

5. Suppose $A$ (resp. $B)$ is an object of $\mathcal{C}_{\mathcal{O}}^{\text {noeth }}$ with minimal prime ideals $\mathfrak{p}_{1}, \ldots, \mathfrak{p}_{r}$ (resp. $\left.\mathfrak{q}_{1}, \ldots, \mathfrak{q}_{s}\right)$. Suppose also that the rings $A / \mathfrak{p}_{i}$ and $B / \mathfrak{q}_{j}$ are all flat over $\mathcal{O}$ and geometrically integral. Then the distinct minimal primes of $A \widehat{\otimes}_{\mathcal{O}} B$ are the

$$
\mathfrak{p}_{i}\left(A \widehat{\otimes}_{\mathcal{O}} B\right)+\mathfrak{q}_{j}\left(A \widehat{\otimes}_{\mathcal{O}} B\right)
$$

for $i=1, \ldots, r$ and $j=1, \ldots, s$.

6. Suppose $A$ (resp. B) is an object of $\mathcal{C}_{k}^{\text {noeth }}$ with minimal ideals $\mathfrak{p}_{1}, \ldots, \mathfrak{p}_{r}$ (resp. $\left.\mathfrak{q}_{1}, \ldots, \mathfrak{q}_{s}\right)$. Suppose also that the rings $A / \mathfrak{p}_{i}$ and $B / \mathfrak{q}_{j}$ are all geometrically integral. Then the distinct minimal primes of $A \widehat{\otimes}_{\mathcal{O}} B$ are the

$$
\mathfrak{p}_{i}\left(A \widehat{\otimes}_{\mathcal{O}} B\right)+\mathfrak{q}_{j}\left(A \widehat{\otimes}_{\mathcal{O}} B\right)
$$

for $i=1, \ldots, r$ and $j=1, \ldots, s$.

7. Suppose that $A$ and $B$ are two objects of $\mathcal{C}_{\mathcal{O}}^{\text {noeth }}$. Suppose also that for each minimal prime $\mathfrak{p}$ of $A$ (resp. B) the quotient $A / \mathfrak{p}($ resp. $B / \mathfrak{p})$ is flat over $\mathcal{O}$ and geometrically integral. Suppose moreover that for each prime $\mathfrak{q}$ of $A$ (resp. B) which is minimal over $\lambda A$ (resp. $\lambda B)$ the quotient $A / \mathfrak{q}($ resp. B/q) is geometrically integral. Suppose finally that for each prime $\mathfrak{q}$ of $A$ (resp. B) which is minimal over $\lambda A$ (resp. $\lambda B)$, q contains a unique minimal prime of $A$ (resp. $B)$. 
Then each prime of $A \widehat{\otimes}_{\mathcal{O}} B$ which is minimal over $\lambda\left(A \widehat{\otimes}_{\mathcal{O}} B\right)$ contains a unique minimal prime of $A \widehat{\otimes}_{\mathcal{O}} B$.

8. Suppose that $X / \operatorname{Spec} \mathcal{O}$ is a flat scheme of finite type and that $x \in X$ is a closed point with residue field $k$. Then there is a finite extension $L^{\prime} / L$ such that for every minimal prime ideal $\mathfrak{p}$ of $\mathcal{O}_{X, x}^{\wedge} \otimes_{\mathcal{O}} \mathcal{O}_{L^{\prime}}$ the algebra $\left(\mathcal{O}_{X, x}^{\wedge} \otimes_{\mathcal{O}} \mathcal{O}_{L^{\prime}}\right) / \mathfrak{p}$ is flat over $\mathcal{O}_{L^{\prime}}$ and geometrically integral.

9. Suppose that $X /$ Spec $k$ is a scheme of finite type and that $x \in X$ is a closed point with residue field $k$. Then there is a finite extension $k^{\prime} / k$ such that for every minimal prime ideal $\mathfrak{p}$ of $\mathcal{O}_{X, x}^{\wedge} \otimes_{k} k^{\prime}$ the algebra $\left(\mathcal{O}_{X, x}^{\wedge} \otimes_{k} k^{\prime}\right) / \mathfrak{p}$ is geometrically integral.

Proof. The first part is proved in exactly the same way as Lemma 2.6 of Tay]. For the second part note that $A \widehat{\otimes}_{\mathcal{O}} B$ is flat over $A$ (as in the first paragraph of the proof of Lemma 3.4.12 of [Ki]). Thus the Krull dimension of $A \widehat{\otimes}_{\mathcal{O}} B$ equals the Krull dimension of $A$ plus the Krull dimension of

$$
k \otimes_{A}\left(A \widehat{\otimes}_{\mathcal{O}} B\right) \cong k \otimes_{\mathcal{O}} B .
$$

As $B$ is flat over $\mathcal{O}$ this latter quantity is one less than the Krull dimension of $B$. The third part is proved in exactly the same way as Lemma 3.4.12 of [Ki], with the reference to $[\mathrm{deJ}]$ replaced by a reference to the first part of this lemma.

For the fourth part note that $A[[T]]$ and $B[[T]]$ are flat over $k[[T]]$, geometrically integral (resp. irreducible) objects of $\mathcal{C}_{k[[T]]}^{\text {noeth }}$. (If $L^{\prime} / k((T))$ is a finite extension then $\mathcal{O}_{L^{\prime}}$ is a complete local Dedekind domain, and hence isomorphic to $k^{\prime}[[S]]$ for some finite extension $k^{\prime} / k$ (see $\left.[\mathrm{M},(28 . J)]\right)$. Thus $A[[T]] \otimes_{k[[T]]} \mathcal{O}_{L^{\prime}} \cong\left(A \otimes_{k} k^{\prime}\right)[[S]]$ is integral (resp. irreducible).) Thus by the second part $\left(A[[T]] \widehat{\otimes}_{k[[T]]} B[[T]]\right) \otimes_{k[[T]]}$ $k^{\prime}[[T]]$ is an integral domain (respectively has irreducible spectrum) for any finite extension $k^{\prime} / k$. However $\left(A[[T]] \widehat{\otimes}_{k[[T]]} B[[T]]\right) \otimes_{k[[T]]} k^{\prime}[[T]] \cong\left(\left(A \widehat{\otimes}_{k} B\right) \otimes_{k} k^{\prime}\right)[[T]]$ (one can write explicit maps in both directions), and so we deduce that $\left(A \widehat{\otimes}_{k} B\right)$ $\otimes_{k} k^{\prime}$ is an integral domain (resp. has irreducible spectrum) for any finite extension $k^{\prime} / k$.

The fifth and sixth parts are proved in the same way, so we will just describe the proof of the fifth part. There are exact sequences

$$
(0) \rightarrow \mathfrak{p}_{i}\left(A \widehat{\otimes}_{\mathcal{O}} B\right) \rightarrow A \widehat{\otimes}_{\mathcal{O}} B \rightarrow\left(A / \mathfrak{p}_{i}\right) \widehat{\otimes}_{\mathcal{O}} B \rightarrow(0)
$$

and

$$
(0) \rightarrow \mathfrak{q}_{j}\left(\left(A / \mathfrak{p}_{i}\right) \widehat{\otimes}_{\mathcal{O}} B\right) \rightarrow\left(A / \mathfrak{p}_{i}\right) \widehat{\otimes}_{\mathcal{O}} B \rightarrow\left(A / \mathfrak{p}_{i}\right) \widehat{\otimes}_{\mathcal{O}}\left(B / \mathfrak{q}_{j}\right) \rightarrow(0),
$$

so that

$$
\left(A \widehat{\otimes}_{\mathcal{O}} B\right) /\left(\mathfrak{p}_{i}\left(A \widehat{\otimes}_{\mathcal{O}} B\right)+\mathfrak{q}_{j}\left(A \widehat{\otimes}_{\mathcal{O}} B\right)\right) \stackrel{\sim}{\rightarrow}\left(A / \mathfrak{p}_{i}\right) \widehat{\otimes}_{\mathcal{O}}\left(B / \mathfrak{q}_{j}\right),
$$


and $\mathfrak{p}_{i}\left(A \widehat{\otimes}_{\mathcal{O}} B\right)+\mathfrak{q}_{j}\left(A \widehat{\otimes}_{\mathcal{O}} B\right)$ is a prime ideal of $A \widehat{\otimes}_{\mathcal{O}} B$. On the other hand if $\mathfrak{P}$ is any prime ideal of $A \widehat{\otimes}_{\mathcal{O}} B$ then the contraction of $\mathfrak{P}$ to $A$ contains some $\mathfrak{p}_{i}$ and the contraction to $B$ some $\mathfrak{q}_{j}$ so that $\mathfrak{P} \supset \mathfrak{p}_{i}\left(A \widehat{\otimes}_{\mathcal{O}} B\right)+\mathfrak{q}_{j}\left(A \widehat{\otimes}_{\mathcal{O}} B\right)$. Finally we must show that no $\mathfrak{p}_{i}\left(A \widehat{\otimes}_{\mathcal{O}} B\right)+\mathfrak{q}_{j}\left(A \widehat{\otimes}_{\mathcal{O}} B\right)$ contains another. This follows because the map $A / \mathfrak{p}_{i} \rightarrow\left(A / \mathfrak{p}_{i}\right) \widehat{\otimes}_{\mathcal{O}}\left(B / \mathfrak{q}_{j}\right)$ is injective (because the algebra $\left(A / \mathfrak{p}_{i}\right) \widehat{\otimes}_{\mathcal{O}}\left(B / \mathfrak{q}_{j}\right)$ is flat over $A / \mathfrak{p}_{i}$ by the argument of the first paragraph of the proof of Lemma 3.4.12 of [Ki]).

For the seventh part note that any prime of $A \widehat{\otimes}_{\mathcal{O}} B$ which is minimal over $\lambda\left(A \widehat{\otimes}_{\mathcal{O}} B\right)$ has the form $\mathfrak{p}^{\prime}\left(A \widehat{\otimes}_{\mathcal{O}} B\right)+\mathfrak{q}^{\prime}\left(A \widehat{\otimes}_{\mathcal{O}} B\right)$, where $\mathfrak{p}^{\prime}$ (resp. $\left.\mathfrak{q}^{\prime}\right)$ is a prime of $A$ (resp. $B$ ) minimal over $\lambda A$ (resp. $\lambda B$ ). Similarly any minimal prime of $A \widehat{\otimes}_{\mathcal{O}} B$ has the form $\mathfrak{p}\left(A \widehat{\otimes}_{\mathcal{O}} B\right)+\mathfrak{q}\left(A \widehat{\otimes}_{\mathcal{O}} B\right)$, where $\mathfrak{p}$ (resp. $\left.\mathfrak{q}\right)$ is a minimal prime of $A$ (resp. $B$ ). It suffices to show that if

$$
\mathfrak{p}^{\prime}\left(A \widehat{\otimes}_{\mathcal{O}} B\right)+\mathfrak{q}^{\prime}\left(A \widehat{\otimes}_{\mathcal{O}} B\right) \supset \mathfrak{p}\left(A \widehat{\otimes}_{\mathcal{O}} B\right)+\mathfrak{q}\left(A \widehat{\otimes}_{\mathcal{O}} B\right)
$$

then $\mathfrak{p}^{\prime} \supset \mathfrak{p}$ and $\mathfrak{q}^{\prime} \supset \mathfrak{q}$. However as

$$
A / \mathfrak{p}^{\prime} \hookrightarrow\left(A \widehat{\otimes}_{\mathcal{O}} B\right) /\left(\mathfrak{p}^{\prime}\left(A \widehat{\otimes}_{\mathcal{O}} B\right)+\mathfrak{q}^{\prime}\left(A \widehat{\otimes}_{\mathcal{O}} B\right)\right)
$$

we see that the composite

$$
\begin{aligned}
A / \mathfrak{p} \rightarrow\left(A \widehat{\otimes}_{\mathcal{O}} B\right) /\left(\mathfrak{p}\left(A \widehat{\otimes}_{\mathcal{O}} B\right)\right. & \left.+\mathfrak{q}\left(A \widehat{\otimes}_{\mathcal{O}} B\right)\right) \\
\rightarrow & \left(A \widehat{\otimes}_{\mathcal{O}} B\right) /\left(\mathfrak{p}^{\prime}\left(A \widehat{\otimes}_{\mathcal{O}} B\right)+\mathfrak{q}^{\prime}\left(A \widehat{\otimes}_{\mathcal{O}} B\right)\right)
\end{aligned}
$$

factors through $A / \mathfrak{p}^{\prime}$, i.e. $\mathfrak{p}^{\prime} \supset \mathfrak{p}$. Similarly $\mathfrak{q}^{\prime} \supset \mathfrak{q}$.

Again the eighth and ninth parts are proved in the same way and we will just describe the eighth part. Suppose $L^{\prime \prime} / L$ is a finite extension and that $x^{\prime \prime}$ is the unique closed point of $X \times \operatorname{Spec} \mathcal{O}_{L^{\prime \prime}}$ above $x$. From the proof of Lemma 2.7 of

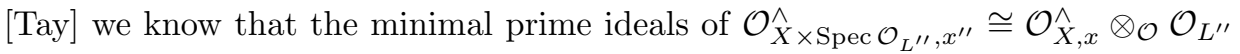
are exactly the kernels of the maps

$$
\mathcal{O}_{X \times \operatorname{Spec}}^{\wedge} \mathcal{O}_{L^{\prime \prime}, x^{\prime \prime}} \rightarrow \mathcal{O}_{\tilde{X}_{i}, y}^{\wedge}
$$

where $\widetilde{X}_{i}$ runs over the normalizations of the irreducible components of $X \times_{\mathcal{O}} \mathcal{O}_{L^{\prime \prime}}$ and where $y$ runs over the points of $\widetilde{X}_{i}$ over $x^{\prime \prime}$. Now there is a finite extension $L^{\prime} / L$ such that if $\widetilde{X}_{i} / \mathcal{O}_{L^{\prime}}$ are the normalizations of the irreducible components of $X \times \operatorname{Spec} \mathcal{O}_{L^{\prime}}$ then for any finite extension $L^{\prime \prime} / L^{\prime}$ the $\widetilde{X}_{i} \times \operatorname{Spec} \mathcal{O}_{L^{\prime \prime}}$ are the normalizations of the irreducible components of $X \times \operatorname{Spec} \mathcal{O}_{L^{\prime \prime}}$. (If $X=\operatorname{Spec} A$ let $\widetilde{B}$ denote the integral closure of the image of $A \otimes_{\mathcal{O}} \mathcal{O}_{\bar{L}}$ in its total quotient ring. Let $\widetilde{B}=\left(A \otimes_{\mathcal{O}} \mathcal{O}_{\bar{L}}\right)\left[b_{1}, \ldots, b_{r}\right]$ and let $f_{1}, \ldots, f_{r}$ be monic polynomials in $\left(A \otimes_{\mathcal{O}} \mathcal{O}_{\bar{L}}\right)[T]$ such that $f_{i}\left(b_{i}\right)=0$ for $i=1, \ldots, r$. Let $L^{\prime} / L$ be a finite extension such that the coefficients of each $f_{i}$ lie in $A \otimes_{\mathcal{O}} \mathcal{O}_{L^{\prime}}$. This field $L^{\prime}$ suffices.) Let $x^{\prime}$ 
be the unique point of $X \times \operatorname{Spec} \mathcal{O}_{L^{\prime}}$ above $x$. We may further assume that every point of each $\widetilde{X}_{i}$ over $x^{\prime}$ is actually defined over $\mathcal{O}_{L^{\prime}} / \lambda^{\prime}$ (where $\lambda^{\prime}$ is the maximal ideal of $\left.\mathcal{O}_{L^{\prime}}\right)$. Let $\mathfrak{p}_{i, y}$ denote the kernel of

$$
\mathcal{O}_{X \times \operatorname{Spec}}^{\wedge} \mathcal{O}_{L^{\prime}, x^{\prime}} \rightarrow \mathcal{O}_{\widetilde{X}_{i}, y}^{\wedge}
$$

If $L^{\prime \prime} / L^{\prime}$ is a finite extension and $x^{\prime \prime}$ is the unique point of $X \times \operatorname{Spec} \mathcal{O}_{L^{\prime \prime}}$ above $x$ then we see that $\mathfrak{p}_{i, y} \mathcal{O}_{X \otimes \operatorname{Spec}}^{\wedge} \mathcal{O}_{L^{\prime \prime}, x^{\prime \prime}}=\mathfrak{p}_{i, y}\left(\mathcal{O}_{X \times \operatorname{Spec}}^{\wedge} \mathcal{O}_{L^{\prime}, x^{\prime}} \otimes_{\mathcal{O}_{L^{\prime}}} \mathcal{O}_{L^{\prime \prime}}\right)$ is the kernel of

$$
\mathcal{O}_{X \otimes \operatorname{Spec}}^{\wedge} \mathcal{O}_{L^{\prime}, x^{\prime}} \otimes_{\mathcal{O}_{L^{\prime}}} \mathcal{O}_{L^{\prime \prime}} \rightarrow \mathcal{O}_{\widetilde{X}_{i}, y}^{\wedge} \otimes_{\mathcal{O}_{L^{\prime}}} \mathcal{O}_{L^{\prime \prime}} \cong \mathcal{O}_{\widetilde{X}_{i} \times \operatorname{Spec}}^{\wedge} \mathcal{O}_{L^{\prime \prime}}, y^{\prime \prime}
$$

where $y^{\prime \prime}$ denotes the unique point of $\widetilde{X}_{i} \times \operatorname{Spec} \mathcal{O}_{L^{\prime \prime}}$ above $y$. Thus the ideal $\mathfrak{p}_{i, y}\left(\mathcal{O}_{X \times \operatorname{Spec}}^{\wedge} \mathcal{O}_{L^{\prime}, x^{\prime}} \otimes_{\mathcal{O}_{L^{\prime}}} \mathcal{O}_{L^{\prime \prime}}\right)$ is prime and

$$
\left(\mathcal{O}_{X \times \operatorname{Spec}}^{\wedge} \mathcal{O}_{L^{\prime}, x^{\prime}} / \mathfrak{p}_{i, y}\right) \otimes_{\mathcal{O}_{L^{\prime}}} \mathcal{O}_{L^{\prime \prime}}
$$

is integral.

Now we return to the properties of

$$
R_{\left\{\chi_{v}\right\}, \infty}^{\square_{T}}=\left(\widehat{\bigotimes_{v \in T}} R_{v}^{\text {loc }} / \mathcal{I}_{v}\right)\left[\left[Y_{1}, \ldots, Y_{q^{\prime}}\right]\right] .
$$

We can calculate the Krull dimension of all irreducible components of this ring using the second part of the previous lemma and the calculation of the Krull dimension of all irreducible components of the $R_{v}^{\text {loc }} / \mathcal{I}_{v}$ in Section 2.4 of [CHT] and Proposition 3.1 of [Tay] (and the fact, also proved there, that the generic point of all these components has characteristic 0$)$. For $v \notin R \cup S(B)_{1}$ it is shown in Section 2.4 of [CHT] that $R_{v}^{\text {loc }} / \mathcal{I}_{v}$ is a power series ring over $\mathcal{O}$ and hence geometrically integral. If $v \in S(B)_{1}$ then Proposition 3.1 of Tay shows that $R_{v}^{\text {loc }} / \mathcal{I}_{v}$ is irreducible, and as we can replace $\mathcal{O}$ by the ring of integers of any finite extension of $L$ in that proposition, it is in fact geometrically irreducible. If $v \in R$ and all the $\chi_{v, i}$ are distinct for $i=1, \ldots, n$ then it follows from Proposition 3.1 of Tay that $R_{v}^{\text {loc }} / \mathcal{I}_{v}$ is geometrically irreducible (again because in that proposition we can replace $\mathcal{O}$ by the ring of integers of any finite extension $\left.L^{\prime} / L\right)$. We deduce that if for all $v \in R$ the characters $\chi_{v, i}$ for $i=1, \ldots, n$ are distinct then $R_{\left\{\chi_{v}\right\}, \infty}^{\square_{T}}$ is irreducible.

We will only prove the last assertion of the first paragraph on page 218 of Tay under the additional assumptions that $L$ is large enough so that for each $v \in R \cup S(B)_{1}$ and each minimal prime $\mathfrak{p}$ of $R_{v}^{\text {loc }} / \mathcal{I}_{v}$ (resp. of $R_{v}^{\text {loc }} /\left(\lambda, \mathcal{I}_{v}\right)$ ) the $\operatorname{ring}\left(R_{v}^{\text {loc }} / \mathcal{I}_{v}\right) / \mathfrak{p}$ (resp. $\left.\left(R_{v}^{\text {loc }} /\left(\lambda, \mathcal{I}_{v}\right)\right) / \mathfrak{p}\right)$ is geometrically integral. This assumption is harmless because we can make a finite extension of $L$ before proving Theorem 4.1 
of Tay] (i.e. Theorem 4.1 for one $L$ implies the same theorem for all other possible $L$ 's). In this case the last assertion of the first paragraph on page 218 of Tay follows from part seven of the above lemma.

\section{[HSBT]}

T.B.-L. has pointed out that in Theorem 3.3 of [HSBT] we should have assumed that $r$ has multiplier $\epsilon_{l}^{1-n}$. The assumptions of the theorem as stated do not seem to be sufficient to conclude that the image $\bar{r}\left(\operatorname{Gal}\left(\bar{F} / F_{1}\right)\right)$ is big. It will be in the case that $\bar{r}$ is surjective, and so Theorem B of the introduction of [HSBT] is correct as stated. See Theorems 7.5 and 7.6 of this paper for results for other multipliers.

\section{$\S 4$. The Dwork family I}

In this section we will follow the arguments of Section 4 of GHK in a slightly more general setting.

Let $N>n+1$ denote positive integers with $n$ even and $N$ odd. (The case $N=n+1$, which was treated in [HSBT and [GHK, is very similar, but for simplicity of exposition we exclude it here.) Recall that $\mathbb{Z}\left[\zeta_{N}\right]^{+}$denotes the subring of $\mathbb{Z}\left[\zeta_{N}\right]$ consisting of elements fixed by complex conjugation. Let $T_{0}$ denote $\mathbb{P}^{1}-$ $\left(\{\infty\} \cup \mu_{N}\right) / \mathbb{Z}[1 / N]$ with coordinate $t$. Let $Y \subset \mathbb{P}^{N-1} \times T_{0}$ denote the family of hypersurfaces

$$
X_{1}^{N}+X_{2}^{N}+\cdots+X_{N}^{N}=N t X_{1} X_{2} \ldots X_{N}
$$

where $t$ is the coordinate on $T_{0}$. Thus $\pi: Y \rightarrow T_{0}$ is smooth of relative dimension $N-2$. Let $\varsigma$ denote the automorphism of $Y$ with $\varsigma^{*} X_{j}=X_{N+1-j}$. Let $H=\mu_{N}^{N} / \mu_{N}$ (diagonal embedding) and let $H_{0}$ be the subgroup

$$
\left\{\left(\xi_{1}, \ldots, \xi_{N}\right) \in \mu_{N}^{N}: \xi_{1} \ldots \xi_{N}=1\right\} / \mu_{N} \subset H .
$$

The scheme $Y \times \operatorname{Spec} \mathbb{Z}\left[1 / N, \zeta_{N}\right]$ has a natural action of the group $H$, given by

$$
\left(\xi_{1}, \ldots, \xi_{N}\right)\left(X_{1}: \cdots: X_{N}\right)=\left(\xi_{1} X_{1}: \cdots: \xi_{N} X_{N}\right),
$$

compatible with the action on $T_{0}$ given by

$$
\left(\xi_{1}, \ldots, \xi_{N}\right) t=\left(\xi_{1} \ldots \xi_{N}\right)^{-1} t .
$$

Thus $H_{0}$ acts on $Y$ over $T_{0} \times \operatorname{Spec} \mathbb{Z}\left[1 / N, \zeta_{N}\right]$. Consider the idempotent

$$
\begin{aligned}
e=\frac{1}{2 \# H_{0}}(\varsigma+1) \sum_{\xi \in H_{0}}\left(\xi_{1}^{1} \xi_{2}^{2} \ldots \xi_{(N-n-1) / 2}^{(N-n-1) / 2} \xi_{(N+n+3) / 2}^{(N+n+1) / 2} \ldots \xi_{N}^{N-1}\right. & \\
& \left.+\xi_{1}^{-1} \xi_{2}^{-2} \ldots \xi_{(N-n-1) / 2}^{-(N-n-1) / 2} \xi_{(N+n+3) / 2}^{(N-n-1) / 2} \ldots \xi_{N}^{1}\right) \xi
\end{aligned}
$$


in the group algebra over $\mathbb{Z}\left[\zeta_{N}, 1 /(2 N)\right]^{+}$of the semidirect product of $H_{0}$ by $\{1, \varsigma\}$. The idempotent $e$ defines a self-correspondence $[e]$ with coefficients from $\mathbb{Z}\left[1 /(2 N), \zeta_{N}\right]^{+}$on $Y$ over $T_{0} \times \operatorname{Spec} \mathbb{Z}\left[1 / N, \zeta_{N}\right]^{+}$.

If $\lambda \mid l$ is a prime of $\mathbb{Z}\left[1 /(2 N), \zeta_{N}\right]^{+}$(resp. if $\mathfrak{n}$ is a non-zero ideal of $\left.\mathbb{Z}\left[1 /(2 N), \zeta_{N}\right]^{+}\right)$then we define a lisse sheaf $V_{\lambda} /\left(T_{0} \times \operatorname{Spec} \mathbb{Z}\left[1 /(2 N l), \zeta_{N}\right]^{+}\right)_{\text {et }}$ (resp. $V[\mathfrak{n}] /\left(T_{0} \times \operatorname{Spec} \mathbb{Z}\left[1 /(2 N(\mathbf{N n})), \zeta_{N}\right]^{+}\right)$et $)$by

$$
V_{\lambda}=[e]^{*} R^{N-2} \pi_{*} \mathbb{Z}\left[1 /(2 N), \zeta_{N}\right]_{\lambda}^{+}
$$

(resp.

$$
\left.V[\mathfrak{n}]=[e]^{*} R^{N-2} \pi_{*} \mathbb{Z}\left[1 /(2 N), \zeta_{N}\right]^{+} / \mathfrak{n}\right) .
$$

Similarly define lisse sheaves $U_{\lambda}$ over $\left(T_{0} \times \operatorname{Spec} \mathbb{Z}\left[1 /(2 N l), \zeta_{N}\right]\right)_{\text {et }}$ and $U[\mathfrak{n}]$ over $\left(T_{0} \times \operatorname{Spec} \mathbb{Z}\left[1 /(2 N(\mathbf{N n})), \zeta_{N}\right]\right)_{\text {et }}$ to be the direct summands of the sheaves $R^{N-2} \pi_{*} \mathbb{Z}\left[1 /(2 N), \zeta_{N}\right]_{\lambda}$ and $R^{N-2} \pi_{*} \mathbb{Z}\left[1 /(2 N), \zeta_{N}\right] / \mathfrak{n}$ where $\xi \in H_{0}$ acts as

$$
\xi_{1}^{1} \xi_{2}^{2} \ldots \xi_{(N-n-1) / 2}^{(N-n-1) / 2} \xi_{(N+n+3) / 2}^{(N+n+1) / 2} \ldots \xi_{N}^{N-1} .
$$

Then $H_{0}$-equivariant projection defines isomorphisms

$$
V_{\lambda} \otimes_{\mathbb{Z}\left[1 /(2 N), \zeta_{N}\right]_{\lambda}^{+}} \mathbb{Z}\left[1 /(2 N), \zeta_{N}\right]_{\lambda} \stackrel{\sim}{\rightarrow} U_{\lambda}
$$

over $\left(T_{0} \times \operatorname{Spec} \mathbb{Z}\left[1 /(2 N l), \zeta_{N}\right]\right)_{\text {et }}$, and

$$
V[\mathfrak{n}] \otimes_{\mathbb{Z}\left[1 /(2 N), \zeta_{N}\right]^{+} / \mathfrak{n}} \mathbb{Z}\left[1 /(2 N), \zeta_{N}\right] / \mathfrak{n} \stackrel{\sim}{\rightarrow} U[\mathfrak{n}]
$$

over $\left(T_{0} \times \operatorname{Spec} \mathbb{Z}\left[1 /(2 N(\mathbf{N n})), \zeta_{N}\right]\right)_{\text {et }}$.

The action of $H_{0}$ on the fibre $Y_{0}$ extends to an action of $H$. Thus we can decompose the fibres

$$
U_{\lambda, 0}=\bigoplus_{i=1}^{N} U_{i, \lambda} \quad \text { and } \quad U[\mathfrak{n}]_{0}=\bigoplus_{i=1}^{N} U_{i}[\mathfrak{n}]
$$

where $U_{i, \lambda}$ (resp. $\left.U_{i}[\mathfrak{n}]\right)$ is the direct summand where $\xi \in H$ acts by

$$
\xi_{1}^{1+i} \xi_{2}^{2+i} \ldots \xi_{(N-n-1) / 2}^{(N-n-1) / 2+i}\left(\xi_{(N-n+1) / 2} \ldots \xi_{(N+n+1) / 2}\right)^{i} \xi_{(N+n+3) / 2}^{(N+n+1) / 2+i} \ldots \xi_{N}^{N-1+i}
$$

Lemma 4.1. $1 . U_{i, \lambda}$ (resp. $\left.U_{i}[\mathfrak{n}]\right)$ is free rank 1 over $\mathbb{Z}\left[\zeta_{N}, 1 /(2 N)\right]_{\lambda}$ (resp. $\left.\mathbb{Z}\left[\zeta_{N}, 1 /(2 N)\right] / \mathfrak{n}\right)$ for $(N+1-n) / 2 \leq i \leq(N-1+n) / 2$, and otherwise it is trivial. Moreover if $\mathfrak{n}=\prod_{\lambda} \lambda^{a_{\lambda}}$ then

$$
U_{i}[\mathfrak{n}] \cong \prod_{\lambda} U_{i, \lambda} / \lambda^{a_{\lambda}}
$$


2. The sheaf $V_{\lambda}$ (resp. $\left.V[\mathfrak{n}]\right)$ is locally free rank $n$ over $\mathbb{Z}\left[\zeta_{N}, 1 /(2 N)\right]_{\lambda}^{+}$(resp. $\left.\mathbb{Z}\left[\zeta_{N}, 1 /(2 N)\right]^{+} / \mathfrak{n}\right)$. Moreover if $\mathfrak{n}=\prod_{\lambda} \lambda^{a_{\lambda}}$ then

$$
V[\mathfrak{n}] \cong \prod_{\lambda} V_{\lambda} / \lambda^{a_{\lambda}}
$$

Proof. The first part follows by the method of proof of Proposition I.7.4 of [DMOS]. The second part need only be checked on the fibre at 0 , where it follows from the first part.

The cup product induces perfect alternating pairings

$$
V_{\lambda}((N-1-n) / 2) \times V_{\lambda}((N-1-n) / 2) \rightarrow \mathbb{Z}\left[\zeta_{N}, 1 /(2 N)\right]_{\lambda}^{+}(1-n)
$$

and

$$
V[\mathfrak{n}]((N-1-n) / 2) \times V[\mathfrak{n}]((N-1-n) / 2) \rightarrow\left(\mathbb{Z}\left[\zeta_{N}, 1 /(2 N)\right]^{+} / \mathfrak{n}\right)(1-n) .
$$

Now let $F / \mathbb{Q}\left(\zeta_{N}\right)^{+}$be a finite extension and $\mathfrak{n}$ be a non-zero ideal of $\mathbb{Z}\left[\zeta_{N}, 1 /(2 N)\right]^{+}$. Suppose that $W$ is a finite free $\mathbb{Z}\left[\zeta_{N}, 1 /(2 N)\right]^{+} / \mathfrak{n}$-module of rank $n$ with a continuous action of $\operatorname{Gal}(\bar{F} / F)$ and a perfect alternating pairing

$$
\langle,\rangle_{W}: W \times W \rightarrow\left(\mathbb{Z}\left[\zeta_{N}, 1 /(2 N)\right]^{+} / \mathfrak{n}\right)(1-n)
$$

such that

$$
\left\langle g w_{1}, g w_{2}\right\rangle=g\left\langle w_{1}, w_{2}\right\rangle
$$

for all $g \in \operatorname{Gal}(\bar{F} / F)$ and all $w_{1}, w_{2} \in W$. Then $W$ can be thought of as a lisse sheaf on $(\operatorname{Spec} F)_{\text {et }}$. Consider the functor which sends a scheme $S /\left(T_{0} \times_{\mathbb{Z}[1 / N]} F\right)$ to the set of isomorphisms $W_{S} \stackrel{\sim}{\rightarrow} V[\mathfrak{n}]((N-1-n) / 2)_{S}$. It is represented by a finite etale cover $T_{W} /\left(T_{0} \times_{\mathbb{Z}[1 / N]} F\right)$.

The only purpose of the rest of this section is to prove the next proposition. A reader willing to accept it without proof can pass directly to the next section. The proof of the proposition is transcendental. It will be complete once we have established Corollaries 4.5 and 4.8 below.

Proposition 4.2. Keep the above notation and assumptions.

1. If $\mathfrak{n}$ is square free and if no two distinct prime factors of $\mathfrak{n}$ have the same residue characteristic then $T_{W}$ is geometrically connected.

2. If $t \in T_{0}(\mathbb{C})$ then the image of a generator of monodromy at infinity in $T_{0}(\mathbb{C})$ in $S p\left(V_{\lambda}\right) \subset G L_{n}\left(\mathbb{Q}\left(\zeta_{N}\right)_{\lambda}^{+}\right)$has minimal polynomial $(X-1)^{n}$, i.e. it is unipotent with just one Jordan block. 
Fix $\tau_{0}: \mathbb{Q}\left(\zeta_{N}\right) \hookrightarrow \mathbb{C}$ so that $\tau_{0}\left(\zeta_{N}\right)=e^{2 \pi i / N}$. Let $V_{B} / T_{0}(\mathbb{C})$ denote the locally constant sheaf

$$
V_{B}=[e]^{*} R^{N-2} \pi_{*} \mathbb{Z}\left[1 /(2 N), \zeta_{N}\right]^{+} .
$$

The sheaf $V_{B}$ has a natural perfect alternating pairing

$$
\langle,\rangle: V_{B} \times V_{B} \rightarrow \mathbb{Z}\left[\zeta_{N}, 1 /(2 N)\right]^{+} .
$$

Also let $U_{B} / T_{0}(\mathbb{C})$ denote the direct summand of $R^{N-2} \pi_{*} \mathbb{Z}\left[1 /(2 N), \zeta_{N}\right]$ where $\xi \in H_{0}$ acts as

$$
\xi_{1}^{1} \xi_{2}^{2} \ldots \xi_{(N-n-1) / 2}^{(N-n-1) / 2} \xi_{(N+n+3) / 2}^{(N+n+1) / 2} \ldots \xi_{N}^{N-1} .
$$

Thus projection again gives an isomorphism

$$
V_{B} \otimes_{\mathbb{Z}\left[1 /(2 N), \zeta_{N}\right]}+\mathbb{Z}\left[1 /(2 N), \zeta_{N}\right] \stackrel{\sim}{\rightarrow} U_{B}
$$

The sheaf $V_{B, \lambda}$ (resp. $V_{B} / \mathfrak{n} V_{B}$, resp. $U_{B, \lambda}$, resp. $U_{B} / \mathfrak{n}$ ) is the Betti sheaf corresponding to the base change to $\left(T_{0} \times{ }_{\operatorname{Spec}} \mathbb{Z}\left[\zeta_{N}, 1 /(N l)\right]^{+}, \tau_{0}^{*} \operatorname{Spec} \mathbb{C}\right)$ et (resp.

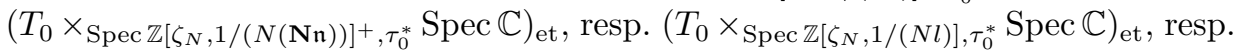
$\left.\left(T_{0} \times_{\operatorname{Spec}} \mathbb{Z}\left[\zeta_{N}, 1 /(N(\mathbf{N} \mathfrak{n}))\right], \tau_{0}^{*} \operatorname{Spec} \mathbb{C}\right)_{\text {et }}\right)$ of $V_{\lambda}$ (resp. $V[\mathfrak{n}]$, resp. $U_{\lambda}$, resp. $\left.U[\mathfrak{n}]\right)$.

Let $\widetilde{T}_{0}$ denote $\mathbb{P}^{1}-\{0,1, \infty\}$ with coordinate $\widetilde{t}$. We will consider $T_{0}-\{0\}$ as a scheme over $\widetilde{T}_{0}$ via $\widetilde{t}=t^{N}$. Also let $\widetilde{Y} \subset \mathbb{P}^{N-1} \times \widetilde{T}_{0}$ denote the family of hypersurfaces

$$
\tilde{X}_{1}^{N}+\cdots+\widetilde{X}_{(N-1) / 2}^{N}+\widetilde{t}^{-1} \widetilde{X}_{(N+1) / 2}^{N}+\widetilde{X}_{(N+3) / 2}^{N}+\cdots+\widetilde{X}_{N}^{N}=N \widetilde{X}_{1} \widetilde{X}_{2} \ldots \widetilde{X}_{N} .
$$

Thus $\tilde{\pi}: \widetilde{Y} \rightarrow \widetilde{T}_{0}$ is smooth of relative dimension $N-2$. Moreover the pull-back of $\widetilde{Y}$ to $T_{0}-\{0\}$ is isomorphic to $Y$ via $\widetilde{X}_{(N+1) / 2}=t X_{(N+1) / 2}$ and $\widetilde{X}_{j}=X_{j}$ for $j \neq(N+1) / 2$. Let $\widetilde{\varsigma}$ denote the automorphism of $\widetilde{Y}$ with $\widetilde{\varsigma}^{*} \widetilde{X}_{j}=\widetilde{X}_{N+1-j}$. Then $\varsigma$ and $\widetilde{\varsigma}$ are compatible with the map $\left(Y-Y_{0}\right) \rightarrow \widetilde{Y}$. Let

$$
\widetilde{H}_{0}=\left\{\left(\xi_{1}, \ldots, \xi_{(N-1) / 2}, \xi_{(N+3) / 2}, \ldots, \xi_{N}\right) \in \mu_{N}^{N-1}\right\} / \mu_{N} .
$$

There is an isomorphism $\widetilde{H}_{0} \stackrel{\sim}{\rightarrow} H_{0}$ given by

$$
\begin{aligned}
\left(\xi_{1}, \ldots, \xi_{(N-1) / 2}, \xi_{(N+3) / 2}, \ldots, \xi_{N}\right) & \\
& \mapsto\left(\xi_{1}, \ldots, \xi_{(N-1) / 2},\left(\xi_{1} \ldots \xi_{(N-1) / 2} \xi_{(N+3) / 2} \ldots \xi_{N}\right)^{-1}, \xi_{(N+3) / 2}, \ldots, \xi_{N}\right) .
\end{aligned}
$$

The hypersurface $\widetilde{Y} \times \operatorname{Spec} \mathbb{Z}\left[1 / N, \zeta_{N}\right]$ has a natural action of the group $\widetilde{H}_{0}$, given by

$$
\begin{aligned}
& \left(\xi_{1}, \ldots, \xi_{N}\right)\left(\widetilde{X}_{1}: \ldots: \widetilde{X}_{N}\right) \\
& \quad=\left(\xi_{1} \widetilde{X}_{1}: \ldots: \xi_{(N-1) / 2} \widetilde{X}_{(N-1) / 2}: \widetilde{X}_{(N+1) / 2}: \xi_{(N+3) / 2} \widetilde{X}_{(N+3) / 2}: \ldots: \xi_{N} \widetilde{X}_{N}\right) .
\end{aligned}
$$


This action is compatible with the maps $\widetilde{H}_{0} \stackrel{\sim}{\rightarrow} H_{0}$ and $\left(Y-Y_{0}\right) \rightarrow \widetilde{Y}$ and the action of $H_{0}$ on $Y$. If we embed $\mu_{N} \hookrightarrow H$ by injection onto the $((N+1) / 2)^{\text {th }}$ coordinate, then $\tilde{Y} \times \operatorname{Spec} \mathbb{Q}\left(\zeta_{N}\right)$ is identified with $\left(\left(Y-Y_{0}\right) \times \operatorname{Spec} \mathbb{Q}\left(\zeta_{N}\right)\right) / \mu_{N}$ and $\widetilde{T}_{0} \times \operatorname{Spec} \mathbb{Q}\left(\zeta_{N}\right)$ with $\left(\left(T_{0}-\{0\}\right) \times \operatorname{Spec} \mathbb{Q}\left(\zeta_{N}\right)\right) / \mu_{N}$.

Consider the idempotent

$$
\begin{aligned}
\widetilde{e}=\frac{1}{2 \# \widetilde{H}_{0}}(\widetilde{\varsigma}+1) \sum_{\xi \in \widetilde{H}_{0}}\left(\xi_{1}^{1} \xi_{2}^{2} \ldots \xi_{(N-n-1) / 2}^{(N-n-1) / 2} \xi_{(N+n+3) / 2}^{(N+n+1) / 2} \ldots \xi_{N}^{N-1}\right. & \\
& \left.+\xi_{1}^{-1} \xi_{2}^{-2} \ldots \xi_{(N-n-1) / 2}^{-(N-n-1) / 2} \xi_{(N+n+3) / 2}^{(N-n-1) / 2} \ldots \xi_{N}^{1}\right) \xi
\end{aligned}
$$

in the group algebra over $\mathbb{Z}\left[\zeta_{N}, 1 /(2 N)\right]^{+}$of the semidirect product of $\widetilde{H}_{0}$ by $\{1, \widetilde{\varsigma}\}$. The idempotent $\widetilde{e}$ defines a self-correspondence $[\widetilde{e}]$ with coefficients from $\mathbb{Z}\left[1 /(2 N), \zeta_{N}\right]^{+}$on $\widetilde{Y}$ over $\widetilde{T}_{0} \times \operatorname{Spec} \mathbb{Z}\left[1 / N, \zeta_{N}\right]^{+}$. Let $\widetilde{V}_{B} / \widetilde{T}_{0}(\mathbb{C})$ denote the locally constant sheaf

$$
\widetilde{V}_{B}=[\widetilde{e}]^{*} R^{N-2} \widetilde{\pi}_{*} \mathbb{Z}\left[1 /(2 N), \zeta_{N}\right]^{+} .
$$

The sheaf $\widetilde{V}_{B}$ has a natural perfect alternating pairing

$$
\langle,\rangle: \widetilde{V}_{B} \times \widetilde{V}_{B} \rightarrow \mathbb{Z}\left[\zeta_{N}, 1 /(2 N)\right]^{+} .
$$

Similarly let $\widetilde{U}_{B}$ denote the direct summand of $R^{N-2} \widetilde{\pi}_{*} \mathbb{Z}\left[1 /(2 N), \zeta_{N}\right]$ on which $\xi \in \widetilde{H}_{0}$ acts by

$$
\xi_{1}^{1} \xi_{2}^{2} \ldots \xi_{(N-n-1) / 2}^{(N-n-1) / 2} \xi_{(N+n+3) / 2}^{(N+n+1) / 2} \ldots \xi_{N}^{N-1}
$$

so that projection gives an isomorphism

$$
\widetilde{V}_{B} \otimes_{\mathbb{Z}\left[1 /(2 N), \zeta_{N}\right]}+\mathbb{Z}\left[1 /(2 N), \zeta_{N}\right] \stackrel{\sim}{\rightarrow} \widetilde{U}_{B}
$$

The pull-back of the pair $\left(\widetilde{V}_{B},\langle\rangle,\right)$ from $\widetilde{T}_{0}(\mathbb{C})$ to $T_{0}(\mathbb{C})-\{0\}$ is naturally identified to the pair $\left(V_{B},\langle\rangle,\right)$.

If $\widetilde{t} \in \widetilde{T}_{0}(\mathbb{C})$ then the fundamental group $\pi_{1}\left(\widetilde{T}_{0}(\mathbb{C}), \widetilde{t}\right)$ is generated by elements $\gamma_{0}, \gamma_{1}$ and $\gamma_{\infty}$ subject only to the relation $\gamma_{0} \gamma_{1} \gamma_{\infty}=1$. Moreover $\gamma_{x}$ is a generator of the monodromy group around $x$ for $x=0,1$ or $\infty$. We get a representation

$$
\rho_{\widetilde{t}}: \pi_{1}\left(\widetilde{T}_{0}(\mathbb{C}), \widetilde{t}\right) \rightarrow S p_{n}\left(\widetilde{V}_{B, \widetilde{t}}\right)
$$

Lemma 4.3. Keep the above notation and assumptions.

1. $\rho_{\widetilde{t}}\left(\gamma_{0}\right)$ has characteristic polynomial $\prod_{j=(N-n+1) / 2}^{(N+n-1) / 2}\left(X-\zeta_{N}^{j}\right)$.

2. $\rho_{\widetilde{t}}\left(\gamma_{1}\right)$ has characteristic polynomial $(X-1)^{n}$, and $\operatorname{ker}\left(\rho_{t}\left(\gamma_{1}\right)-1\right)$ has rank at least $n-1$.

3. $\rho_{\tilde{t}}\left(\gamma_{\infty}\right)$ has characteristic polynomial $(X-1)^{n}$. 
Proof. We may work with $\widetilde{U}_{B} \otimes_{\mathbb{Z}\left[1 /(2 N), \zeta_{N}\right]} \mathbb{Q}\left(\zeta_{N}\right)$ instead of $\widetilde{V}_{B}$.

The image of $\gamma_{0}$ in $\mu_{N}=\operatorname{Gal}\left(T_{0}(\mathbb{C})-\{0\}, \widetilde{T}_{0}(\mathbb{C})\right)$ is $\zeta_{N}^{ \pm 1}=\tau_{0}^{-1}\left(e^{ \pm 2 \pi i / N}\right)$. Thus the action of $\gamma_{0}$ on $\widetilde{U}_{B, \widetilde{t}} \otimes \mathbb{Q}\left(\zeta_{N}\right)$ is equivalent to the action of $\zeta_{N}^{ \pm 1}$ on $U_{B, 0} \otimes \mathbb{Q}\left(\zeta_{N}\right)$. The first part now follows from Lemma 4.1 .

As $T_{0}(\mathbb{C})-\{0\} \rightarrow \widetilde{T}(\mathbb{C})$ is etale in a neighbourhood of $t=1$ the second part will follow if we can show the same for the action of a generator of monodromy around 1 on $U_{B, t}$, where $t$ is a preimage of $\widetilde{t}$ in $T_{0}(\mathbb{C})-\{0\}$. Picard-Lefschetz theory (see [SGA7]) gives an $H_{0}$-orbit $\Delta$ of elements of $H^{n-1}\left(Y_{t}(\mathbb{C}), \mathbb{Z}\right)$ and an exact sequence

$$
(0) \rightarrow H^{n-1}\left(Y_{1}(\mathbb{C}), \mathbb{Z}\right) \rightarrow H^{n-1}\left(Y_{t}(\mathbb{C}), \mathbb{Z}\right) \rightarrow \mathbb{Z}^{\Delta} .
$$

If $x \in H^{n-1}\left(Y_{t}(\mathbb{C}), \mathbb{Z}\right)$ maps to $\left(x_{\delta}\right) \in \mathbb{Z}^{\Delta}$ then the monodromy operator sends $x$ to $x \pm \sum_{\delta \in \Delta} x_{\delta} \delta$. As the action of $H_{0}$ commutes with the action of inertia we see that for $\xi \in H_{0}$ we have $(\xi x)_{\delta}=x_{\xi^{-1} \delta}$. Thus picking $\delta_{0} \in \Delta$ we see that $x$ maps to

$$
x \pm \frac{\# \Delta}{\# H_{0}} \sum_{\xi \in H_{0}}\left(\xi^{-1} x\right)_{\delta_{0}} \xi \delta_{0} .
$$

Thus if $x$ transforms under a character $\chi$ under the action of $H_{0}$ it maps to

$$
x \pm x_{\delta_{0}} \frac{\# \Delta}{\# H_{0}} \sum_{\xi \in H_{0}} \chi(\xi)^{-1} \xi \delta_{0} .
$$

Thus $\operatorname{ker}\left(\gamma_{1}-1\right)$ has rank at least $n-1$ on $U_{B, t}$. As $\gamma_{1}$ acts as a symplectic transformation we conclude that it has characteristic polynomial $(X-1)^{n}$.

For the third part one can refer to the combination of Theorem 5.3 of Ka2 and Theorem 8.4.11 of Ka1. However it would seem to us more elegant to find a direct argument which does not go via hypergeometric sheaves.

Write

$$
(X-1)^{n}=X^{n}+A_{1} X^{n-1}+\cdots+A_{n}
$$

and

and

$$
\prod_{j=(N-n+1) / 2}^{(N+n-1) / 2}\left(X-\zeta_{N}^{j}\right)=X^{n}+B_{1} X^{n-1}+\cdots+B_{n}
$$

$$
A=\left(\begin{array}{ccccc}
0 & 0 & \ldots & 0 & -A_{n} \\
1 & 0 & \ldots & 0 & -A_{n-1} \\
0 & 1 & \ldots & 0 & -A_{n-2} \\
& & \ddots & & \\
0 & 0 & \ldots & 1 & -A_{1}
\end{array}\right)
$$


and

$$
B=\left(\begin{array}{ccccc}
0 & 0 & \ldots & 0 & -B_{n} \\
1 & 0 & \ldots & 0 & -B_{n-1} \\
0 & 1 & \ldots & 0 & -B_{n-2} \\
& & \ddots & & \\
0 & 0 & \ldots & 1 & -B_{1}
\end{array}\right) .
$$

Then Theorem 1.1 of $[\mathrm{Le}$ (but see also Theorem 3.5 of $[\mathrm{BH}]$ ) and Proposition 3.3 of $\mathrm{BH}]$ give us the following corollary.

Corollary 4.4. Keep the above notation and assumptions and fix $S p_{n}\left(\widetilde{V}_{B, \tilde{t}}\right) \subset$ $G L_{n}(\mathbb{C})$. As a representation into $G L_{n}(\mathbb{C})$, the representation $\rho_{\tilde{t}}$ is conjugate to one sending $\gamma_{\infty}$ to $A ; \gamma_{1}$ to $B A^{-1}$; and $\gamma_{0}$ to $B^{-1}$. Moreover this representation is irreducible.

Corollary 4.5. Keep the above notation and assumptions. Then $\rho_{\tilde{t}}\left(\gamma_{\infty}\right)$ has minimal polynomial $(X-1)^{n}$, i.e. it has just one Jordan block. Hence the same is true for the image of a generator of monodromy at infinity in $S p\left(V_{B, t}\right) \subset G L_{n}(\mathbb{C})$ (for any $\left.t \in T_{0}(\mathbb{C})\right)$.

Lemma 4.6. Keep the above notation and assumptions. Suppose that $\lambda$ is a nonzero prime of $\mathbb{Z}\left[\zeta_{N}, 1 /(2 N)\right]^{+}$and let $k(\lambda)$ denote its residue field. Then the image of $\rho_{\tilde{t}} \bmod \lambda$ is $S p\left(\widetilde{V}_{B, t} / \lambda\right)$.

Proof. Let $\rho: \pi_{1}\left(\widetilde{T}_{0}(\mathbb{C}), \widetilde{t}\right) \rightarrow G L_{n}\left(\mathbb{Z}\left[\zeta_{N}, 1 /(2 N)\right]^{+}\right)$be the representation sending $\gamma_{0}$ to $B^{-1}$ and $\gamma_{\infty}$ to $A$. The argument for Proposition 3.3 of [BH] shows that $\rho \bmod \lambda$ is absolutely irreducible. On the other hand, by the last but one corollary $\rho_{\widetilde{t}} \bmod \lambda$ and $\rho \bmod \lambda$ have the same trace and so they have equivalent semisimplifications. Thus $\rho_{\widetilde{t}} \bmod \lambda$ is absolutely irreducible.

Let $\Delta \subset \pi_{1}\left(\widetilde{T}_{0}(\mathbb{C}), \widetilde{t}\right)$ denote the normal subgroup generated by $\gamma_{1}$. Then $\pi_{1}\left(\widetilde{T}_{0}(\mathbb{C}), \widetilde{t}\right) / \Delta$ is cyclic generated by $\gamma_{0} \Delta=\gamma_{\infty}^{-1} \Delta$. Thus the in$\operatorname{dex}\left[\left(\rho_{\tilde{t}} \bmod \lambda\right)\left(\pi_{1}\left(\widetilde{T}_{0}(\mathbb{C}), \widetilde{t}\right)\right):\left(\rho_{\tilde{t}} \bmod \lambda\right)(\Delta)\right]$ divides both the order of $\left(\rho_{\tilde{t}} \bmod \lambda\right)\left(\gamma_{0}\right)$ and $\left(\rho_{\tilde{t}} \bmod \lambda\right)\left(\gamma_{\infty}\right)$, i.e. divides both $N$ and a power of the characteristic of $k(\lambda)$. Thus $\left(\rho_{\tilde{t}} \bmod \lambda\right)\left(\pi_{1}\left(\widetilde{T}_{0}(\mathbb{C}), \widetilde{t}\right)\right)=\left(\rho_{\tilde{t}} \bmod \lambda\right)(\Delta)$ and $\left(\rho_{\widetilde{t}} \bmod \lambda\left(\pi_{1}\left(\widetilde{T}_{0}(\mathbb{C}), \widetilde{t}\right)\right)\right.$ is generated by elements $C$ with characteristic polynomial $(X-1)^{n}$ and $\operatorname{dim} \operatorname{ker}(C-1) \geq n-1$. It follows from the main theorem of [SZ that $\left(\rho_{\widetilde{t}} \bmod \lambda\right)\left(\pi_{1}\left(\widetilde{T}_{0}(\mathbb{C}), \widetilde{t}\right)\right)$ is conjugate in $G L_{n}(k(\lambda))$ to one of the groups $S L_{n}(k), S p_{n}(k)$ or $S U(n, k)$ for some subfield $k \subset k(\lambda)$. (Here $S U(n, k)$ is only defined if $k$ has even degree over its prime subfield and then it equals the subgroup of $S L_{n}(k)$ consisting of the matrices $g$ with $\sigma(g)^{t} g=1_{n}$, where $\sigma$ is the unique field automorphism of $k$ of order exactly 2.) Because 
the image is contained in a conjugate of $S p_{n}(k(\lambda))$ we see that the possibilities $S L_{n}(k(\lambda))$ and $S U(n, k(\lambda))$ cannot occur unless $n=2$. (If $\alpha, \beta \in k(\lambda)$ satisfy $\sigma(\alpha) \alpha=\sigma(\beta) \beta=1$ then the diagonal matrix $\left(\alpha, \beta,(\alpha \beta)^{-1}, 1, \ldots, 1\right)$ lies in $S U(n, k(\lambda))$ but can only be conjugate to an element of $S p_{n}(k(\lambda))$ if one of $\alpha, \beta$ or $\alpha \beta$ equals 1.) Also note that $S p_{2}(k(\lambda))=S L_{2}(k(\lambda))$ and that $\operatorname{tr} S U(2, k(\lambda)) \subset k(\lambda)^{\sigma=1}$. (If $g \in S U(2, k(\lambda))$ then $\operatorname{tr} g=\operatorname{tr} g^{-1}=\operatorname{tr} \sigma(g)=$ $\sigma(\operatorname{tr} g)$.) Thus it suffices to show that the characteristic polynomials of the elements of $\left(\rho_{\widetilde{t}} \bmod \lambda\right)\left(\pi_{1}\left(\widetilde{T}_{0}(\mathbb{C}), \widetilde{t}\right)\right)$ do not all lie in $k[X]$ for some proper subfield $k \subset k(\lambda)$.

Considering just the element $\left(\rho_{\tilde{t}} \bmod \lambda\right)\left(\gamma_{0}\right)$ we see that it suffices to check that if $\alpha \in(\mathbb{Z} / N \mathbb{Z})^{\times}$and

$$
\begin{aligned}
& \{\alpha j: j=(N+1-n) / 2, \ldots,(N-1+n) / 2\} \\
& \quad=\{j: j=(N+1-n) / 2, \ldots,(N-1+n) / 2\} \subset \mathbb{Z} / N \mathbb{Z}
\end{aligned}
$$

then $\alpha= \pm 1$. (See part 1 of Lemma 4.3 for the characteristic polynomial of $\left(\rho_{\tilde{t}} \bmod \lambda\right)\left(\gamma_{0}\right)$.) The case $n=2$ is easy, so we suppose $n>2$. We may assume that $\alpha$ has a representative $a \in \mathbb{Z}$ with $0<a<N / 2$, and we wish to prove $a=1$. Choose $j_{1}$ from amongst $(N+1-n) / 2, \ldots,(N-3+n) / 2$ and $j_{2}$ from amongst $(N+1-n) / 2, \ldots,(N+1) / 2$ with $\alpha j_{1} \equiv j_{2} \bmod N$. (This is possible as $n>2$.) Then $\alpha\left(j_{1}+1\right) \equiv j_{2}+a \bmod N$ and $j_{2}+a \leq N$, so that we must have $j_{2}+a \leq(N-1+n) / 2$, which implies that $a<n$. Now if $j=0, \ldots,(N-1-n) / 2$ and $\alpha^{-1} j \not \equiv(N-1-n) / 2 \bmod N$ then $\alpha\left(\alpha^{-1} j+1\right) \equiv j+a \bmod N$ and $j+a<$ $(N-1+n) / 2$, so that we must have $j+a<(N+1-n) / 2$. Thus indeed we must have $a=1$, and the proof is complete.

Corollary 4.7. Suppose that $t \in T_{0}(\mathbb{C})$. Let $\lambda$ be a non-zero prime of the ring $\mathbb{Z}\left[\zeta_{N}, 1 /(2 N)\right]^{+}$. Then the natural map $\pi_{1}\left(T_{0}(\mathbb{C}), t\right) \rightarrow S p\left(V_{B, t} / \lambda\right)$ is surjective.

Proof. We may reduce to the case $t \neq 0$. Then it suffices to show that the composite

$$
\pi_{1}\left(T_{0}(\mathbb{C})-\{0\}, t\right) \rightarrow \pi_{1}\left(T_{0}(\mathbb{C}), t\right) \rightarrow S p\left(V_{B, t} / \lambda\right)
$$

is surjective. However if $\tilde{t}$ denotes the image of $t$ in $\widetilde{T}_{0}(\mathbb{C})$ then $\pi_{1}\left(T_{0}(\mathbb{C})-\{0\}, t\right)$ is a normal subgroup of $\pi_{1}\left(\widetilde{T}_{0}(\mathbb{C}), \widetilde{t}\right)$ with quotient cyclic of order $N$. As

$$
\pi_{1}\left(\widetilde{T}_{0}(\mathbb{C}), \widetilde{t}\right) \rightarrow S p\left(\widetilde{V}_{B, \widetilde{t}} / \lambda\right) \cong S p\left(V_{B, t} / \lambda\right)
$$

it suffices to show that if $l \nmid 2 N$ is a prime then $S p_{n}\left(\mathbb{F}_{l^{r}}\right)$ has no cyclic quotient of order dividing $N$. However the only cyclic composition factors of $S p_{n}\left(\mathbb{F}_{l^{r}}\right)$ have order 2 or 3 , and a cyclic composition factor of order 3 only occurs if $l^{r}=3$ and $n=2$. 
Corollary 4.8. If $\mathfrak{n}$ is a square-free ideal of $\mathbb{Z}\left[\zeta_{N}, 1 /(2 N)\right]^{+}$such that any two distinct prime factors of $\mathfrak{n}$ have distinct residue characteristics, then the map $\pi_{1}\left(T_{0}(\mathbb{C}), t\right) \rightarrow S p\left(V_{B, t} / \mathfrak{n}\right)$ is surjective.

Proof. Suppose $l>2$ is a prime and $r \in \mathbb{Z}_{>0}$ and either $n>2$ or $l^{r} \neq 3$; then the only non-trivial quotient of $S p_{n}\left(\mathbb{F}_{l^{r}}\right)$ is $P S p_{n}\left(\mathbb{F}_{l^{r}}\right)$, which is an insoluble simple group. On the other hand $S p_{2}\left(\mathbb{F}_{3}\right)$ is soluble. Moreover for positive integers $r, s$ and for primes $l \neq l^{\prime}$ both greater than 2 the groups $P S p_{n}\left(\mathbb{F}_{l^{r}}\right)$ and $P S p_{n}\left(\mathbb{F}_{\left(l^{\prime}\right)}\right)$ are not isomorphic. The corollary then follows from the previous corollary and Goursat's lemma.

One can ask if this corollary remains true for any non-zero ideal $\mathfrak{n}$ of $\mathbb{Z}\left[\zeta_{N}, 1 /(2 N)\right]^{+}$. We have now completed the proof of Proposition 4.2

\section{§5. The Dwork family II}

We now turn to an analysis of the action of Galois groups of local fields on the spaces $V_{\lambda, t}$. First we consider the case that $t$ lies in a $p$-adic field with $p$ different from the residue characteristic of $\lambda$. The next lemma follows from Proposition 4.2 as in the proof of Lemma 1.15 of HSBT.

Lemma 5.1. Suppose that $\mathfrak{q}$ and $\lambda$ are non-zero primes of $\mathbb{Z}\left[\zeta_{N}, 1 /(2 N)\right]^{+}$with distinct residue characteristics. Suppose also that $F / \mathbb{Q}\left(\zeta_{N}\right)_{\mathfrak{q}}^{+}$is a finite extension and that $t \in F$ with $t^{N} \neq 1$.

1. If $t \in \mathcal{O}_{F}$ but $t^{N}-1$ is not in the maximal ideal of $\mathcal{O}_{F}$ then the action of $\operatorname{Gal}(\bar{F} / F)$ on $V_{\lambda, t}$ is unramified.

2. If $t \in F-\mathcal{O}_{F}$ then the action of $\operatorname{Gal}(\bar{F} / F)$ on $V_{\lambda, t}$ is tamely ramified. A generator of tame inertia acts via a unipotent matrix with minimal polynomial $(X-1)^{n}$. A Frobenius lift has characteristic polynomial

$$
(X-\alpha)(X-\alpha \# k(\mathfrak{q})) \ldots\left(X-\alpha(\# k(\mathfrak{q}))^{n-1}\right)
$$

for some $\alpha= \pm 1$.

We next turn to the case that $t$ lies in an $l$-adic field, where $l$ equals the residue characteristic of $\lambda$. The rest of this section is entirely devoted to the proof of Lemma 5.3. A reader who is prepared to take that lemma on trust may simply read its statement and skip to the next section.

The proof of Lemma 5.3 will depend on the use of Fontaine's functors to reduce the statements to questions about de Rham cohomology, so we must first study the de Rham cohomology of the varieties $Y_{t}$. The analysis is complicated 
because we have to work with 'de Rham cohomology with coefficients' and these coefficients are embeddable in the field containing $t$, but there is not a canonical embedding.

The relative de Rham cohomology

$$
\mathcal{H}_{\mathrm{DR}}=\mathcal{H}_{\mathrm{DR}}^{N-2}\left(Y /\left(T_{0} \times_{\mathbb{Z}[1 / N]} \mathbb{Q}\left(\zeta_{N}\right)\right)\right)
$$

is a locally free $\mathcal{O}_{T_{0} \times_{\mathbb{Z}[1 / N]} \mathbb{Q}\left(\zeta_{N}\right)}$-module (for the Zariski toplogy on $T_{0} \times_{\mathbb{Z}[1 / N]}$ $\left.\mathbb{Q}\left(\zeta_{N}\right)\right)$. It comes with a decreasing filtration $F^{j} \mathcal{H}_{\mathrm{DR}}$ by local direct summands (with $F^{0} \mathcal{H}_{\mathrm{DR}}=\mathcal{H}_{\mathrm{DR}}$ ). Moreover there are compatible decompositions

$$
F^{j} \mathcal{H}_{\mathrm{DR}} \otimes_{\mathbb{Q}} \mathbb{Q}\left(\zeta_{N}\right)=\bigoplus_{\sigma \in \operatorname{Gal}\left(\mathbb{Q}\left(\zeta_{N}\right) / \mathbb{Q}\right)} F^{j} \mathcal{H}_{\mathrm{DR}, \sigma},
$$

where

$$
F^{j} \mathcal{H}_{\mathrm{DR}, \sigma}=\left(F^{j} \mathcal{H}_{\mathrm{DR}}\right) \otimes_{\sigma^{-1}, \mathbb{Q}\left(\zeta_{N}\right)} \mathbb{Q}\left(\zeta_{N}\right)
$$

Here we think of the second factor $\mathbb{Q}\left(\zeta_{N}\right)$ as 'coefficients'. Define $U_{\mathrm{DR}}, F^{j} U_{\mathrm{DR}}$, $U_{\mathrm{DR}, \sigma}$ and $F^{j} U_{\mathrm{DR}, \sigma}$ to be the direct summands of $\mathcal{H}_{\mathrm{DR}} \otimes_{\mathbb{Q}} \mathbb{Q}\left(\zeta_{N}\right), F^{j} \mathcal{H}_{\mathrm{DR}} \otimes_{\mathbb{Q}}$ $\mathbb{Q}\left(\zeta_{N}\right), \mathcal{H}_{\mathrm{DR}, \sigma}$ and $F^{j} \mathcal{H}_{\mathrm{DR}, \sigma}$ where $\xi \in H_{0}$ acts by

$$
\xi_{1}^{1} \xi_{2}^{2} \ldots \xi_{(N-n-1) / 2}^{(N-n-1) / 2} \xi_{(N+n+3) / 2}^{(N+n+1) / 2} \ldots \xi_{N}^{N-1}
$$

(acting through the right hand 'coefficient' factor of $\mathbb{Q}\left(\zeta_{N}\right)$ ). Again we can decompose the fibres

$$
U_{\mathrm{DR}, 0}=\bigoplus_{i=1}^{N} U_{i, \mathrm{DR}} \quad \text { and } \quad U_{\mathrm{DR}, \sigma, 0}=\bigoplus_{i=1}^{N} U_{i, \mathrm{DR}, \sigma}
$$

where $U_{i, \mathrm{DR}}$ (resp. $U_{i, \mathrm{DR}, \sigma}$ ) is the direct summand where $\xi \in H$ acts by

$$
\xi_{1}^{1+i} \xi_{2}^{2+i} \ldots \xi_{(N-n-1) / 2}^{(N-n-1) / 2+i}\left(\xi_{(N-n+1) / 2} \ldots \xi_{(N+n+1) / 2}\right)^{i} \xi_{(N+n+3) / 2}^{(N+n+1) / 2+i} \ldots \xi_{N}^{N-1+i}
$$

If $\lambda$ and $v$ are primes of $\mathbb{Z}\left[\zeta_{N}, 1 / N\right]$ both with residue characteristic $l$; if $F / \mathbb{Q}\left(\zeta_{N}\right)_{v}$ is a finite extension; if $t \in T_{0}(F)$; and if $\sigma: F \hookrightarrow \overline{\mathbb{Q}\left(\zeta_{N}\right)} \lambda_{\lambda}$, then

$$
\left(\left(U_{\lambda, t} \otimes_{\mathbb{Z}\left[\zeta_{N}, 1 / N\right]_{\lambda}} \overline{\mathbb{Q}\left(\zeta_{N}\right)_{\lambda}}\right) \otimes_{\sigma, F} B_{\mathrm{DR}}\right)^{\mathrm{Gal}(\bar{F} / F)} \cong U_{\mathrm{DR},\left.\sigma\right|_{\mathbb{Q}\left(\zeta_{N}\right)}, t} \otimes_{F, \sigma} \overline{\mathbb{Q}\left(\zeta_{N}\right)_{\lambda}} .
$$

Similarly for $i=1, \ldots, N$ and $\sigma: \mathbb{Q}\left(\zeta_{N}\right)_{v} \hookrightarrow{\overline{\mathbb{Q}}\left(\zeta_{N}\right)_{\lambda}}_{\lambda}$ we have

$$
\begin{aligned}
& \left(\left(U_{i, \lambda} \otimes_{\mathbb{Z}\left[\zeta_{N}, 1 / N\right]_{\lambda}} \overline{\mathbb{Q}\left(\zeta_{N}\right)_{\lambda}}\right) \otimes_{\sigma, \mathbb{Q}\left(\zeta_{N}\right)_{v}} B_{\mathrm{DR}}\right)^{\operatorname{Gal}\left(\overline{\mathbb{Q}\left(\zeta_{N}\right)_{\lambda}} / \mathbb{Q}\left(\zeta_{N}\right)_{\lambda}\right)} \\
& \cong U_{i, \mathrm{DR}, \sigma} \otimes_{\mathbb{Q}\left(\zeta_{N}\right)_{v}, \sigma} \overline{\mathbb{Q}\left(\zeta_{N}\right)_{\lambda}}
\end{aligned}
$$

If $a \in \mathbb{Z}$ we will write $\bar{a}$ for the integer in the range $1 \leq \bar{a} \leq N$ which is congruent to a modulo $N$. Let $\tau_{0}: \mathbb{Q}\left(\zeta_{N}\right) \hookrightarrow \mathbb{C}$ be such that $\tau_{0}\left(\zeta_{N}\right)=e^{2 \pi i / N}$. 
Lemma 5.2. 1. Suppose that $\sigma^{-1}\left(\zeta_{N}\right)=\zeta_{N}^{a}$. Then $U_{i, \mathrm{DR}, \sigma}=(0)$ unless $(N+1-$ $n) / 2 \leq i \leq(N-1+n) / 2$. If $(N+1-n) / 2 \leq i \leq(N-1+n) / 2$ then $U_{i, \mathrm{DR}, \sigma}$ is a one-dimensional $\mathbb{Q}\left(\zeta_{N}\right)$-vector space and $\mathrm{gr}^{j} U_{i, \mathrm{DR}, \sigma}$ is non-zero only for

$$
j=(N-n-3) / 2+\#\{(N+1-n) / 2 \leq b \leq(N-1+n) / 2: \overline{a b} \leq \overline{a i}\} .
$$

2. $U_{\mathrm{DR}, \sigma}$ is a locally free sheaf of $\mathcal{O}_{T_{0} \times \mathbb{Z}[1 / N]} \mathbb{Q}\left(\zeta_{N}\right)$-modules of rank $n$. If $(N-$ $1-n) / 2 \leq j \leq(N+n-3) / 2$ then $\mathrm{gr}^{j} U_{\mathrm{DR}, \sigma}$ is locally free rank 1 over $\mathcal{O}_{T_{0} \times \mathbb{Z}[1 / N] \mathbb{Q}\left(\zeta_{N}\right)}$. Otherwise it is zero.

Proof. As $\operatorname{gr}^{j} U_{\mathrm{DR}, \sigma}$ is locally free over $\mathcal{O}_{T_{0} \times_{\mathbb{Z}[1 / N]} \mathbb{Q}\left(\zeta_{N}\right)}$, the second part follows from the first. Now consider the first part. The space

$$
\operatorname{gr}^{j} U_{i, \mathrm{DR}, \sigma} \otimes_{\mathbb{Q}\left(\zeta_{N}\right), \tau_{0} \sigma^{-1}} \mathbb{C}
$$

is identified with the space

$H^{j, N-2-j}(Y(\mathbb{C}), \mathbb{C})_{(a(1+i), a(2+i), \ldots, a((N-n-1) / 2+i), a i, \ldots, a i, a((N+n+1) / 2+i), \ldots, a(N-1+i))}$

of Section I.7 of [DMOS] (where we define $Y(\mathbb{C})$ via $\tau_{0}: \mathbb{Q}\left(\zeta_{N}\right) \hookrightarrow \mathbb{C}$ ). By Propositions I.7.4 and I.7.6 of DMOS this is non-zero if and only if none of the entries of $(a(1+i), a(2+i), \ldots, a((N-n-1) / 2+i), a i, \ldots, a i, a((N+n+1) / 2+i), \ldots, a(N-$ $1+i)$ ) are congruent to zero modulo $N$ and

$$
\begin{aligned}
j+1=(\overline{a(1+i)}+ & \overline{a(2+i)}+\cdots+\overline{a((N-n-1) / 2+i)}+\overline{a i}+\cdots \\
& +\overline{a i}+\overline{a((N+n+1) / 2+i)}+\cdots+\overline{a(N-1+i)}) / N .
\end{aligned}
$$

Define $j(0)=(N-n-1) / 2$ and

$$
\begin{aligned}
j(d)=(\overline{a+d}+\overline{2 a+d}+\cdots+\overline{a(N-n-1) / 2+d}+\bar{d}+\cdots \\
+\bar{d}+\overline{a(N+n+1) / 2+d} \cdots+\overline{a(N-1)+d}) / N-1
\end{aligned}
$$

for $d>0$. For $d=1, \ldots, N-1$ we have $j(d)=j(d-1)+1$ if $d-1 \equiv$ ai $\bmod N$ for some $(N+1-n) / 2 \leq i \leq(N-1+n) / 2$, and otherwise $j(d)=j(d-1)$. The first part follows.

Lemma 5.3. Suppose that $\lambda$ is a non-zero prime of $\mathbb{Z}\left[\zeta_{N}, 1 /(2 N)\right]^{+}$which has residue characteristic l. Suppose that $v$ is also a place of $\mathbb{Q}\left(\zeta_{N}\right)^{+}$with residue characteristic l. (The placev might, or might not, equal the place $\lambda$.) Let $F / \mathbb{Q}\left(\zeta_{N}\right)_{v}^{+}$ be a finite extension and let $t \in F$ with $t^{N} \neq 1$.

1. $V_{\lambda, t}((N-1-n) / 2)$ is a de Rham representation of $\mathrm{Gal}(\bar{F} / F)$. Moreover for each continuous embedding $\tau: F \hookrightarrow \overline{\mathbb{Q}\left(\zeta_{N}\right)_{\lambda}^{+}}$the Hodge-Tate numbers of $V_{\lambda, t}((N-$ $1-n) / 2) \otimes_{\mathbb{Z}\left[\zeta_{N}, 1 /(2 N)\right]_{\lambda}^{+}} \overline{\mathbb{Q}\left(\zeta_{N}\right)_{\lambda}^{+}}$with respect to $\tau$ are $\{0,1,2, \ldots, n-1\}$. 
2. If $t \in \mathcal{O}_{F}$ and $t^{N}-1$ is not in the maximal ideal of $\mathcal{O}_{F}$ then $V_{\lambda, t}((N-1-n) / 2)$ is crystalline.

3. If $l \equiv 1 \bmod N$ and $t$ is in the maximal ideal of $\mathcal{O}_{F}$ then $V_{\lambda, t}((N-1-n) / 2)$ is ordinary of weight 0 .

4. If $l \equiv 1 \bmod N$ is an odd prime and if $v$ is a place of $\mathbb{Q}\left(\zeta_{N}\right)$ above $l$ then

$$
V[\lambda]((N-1-n) / 2)_{0} \cong \mathbb{F}_{l} \oplus \mathbb{F}_{l}(-1) \oplus \cdots \oplus \mathbb{F}_{l}(1-n)
$$

as a module for $I_{\mathbb{Q}\left(\zeta_{N}\right)_{v}^{+}}$.

5. If $l \equiv-1 \bmod N$ then

$$
\begin{aligned}
& V[\lambda]((N-1-n) / 2)_{0} \otimes_{\mathbb{Z}\left[\zeta_{N}, 1 /(2 N)\right]^{+} / \lambda} \overline{\mathbb{F}}_{l} \\
& \quad \cong \overline{\mathbb{F}}_{l}\left(\omega_{2}^{1-n}\right) \oplus \overline{\mathbb{F}}_{l}\left(\omega_{2}^{-l+2-n}\right) \oplus \overline{\mathbb{F}}_{l}\left(\omega_{2}^{-2 l+3-n}\right) \oplus \cdots \oplus \overline{\mathbb{F}}_{l}\left(\omega_{2}^{l(1-n)}\right)
\end{aligned}
$$

as a module for $I_{\mathbb{Q}\left(\zeta_{N}\right)_{v}^{+}}$.

Proof. The first part follows from Lemma 5.2 and the comparison isomorphism discussed just before the statement of that lemma. The second part follows as for these values of $t$ the variety $Y_{t}$ has good reduction at $v$.

For the third and fourth parts it suffices to show that if $l \equiv 1 \bmod N$ then

$$
V_{\lambda}((N-1-n) / 2)_{0} \otimes_{\mathbb{Z}\left[\zeta_{N}, 1 /(2 N)\right]_{\lambda}^{+}} \overline{\mathbb{Q}}_{l} \cong \overline{\mathbb{Q}}_{l} \oplus \overline{\mathbb{Q}}_{l}(-1) \oplus \cdots \oplus \overline{\mathbb{Q}}_{l}(1-n)
$$

as modules for $I_{\mathbb{Q}\left(\zeta_{N}\right)_{v}^{+}}$. (Note that for $t$ in the maximal ideal of $\mathcal{O}_{F}$ we have

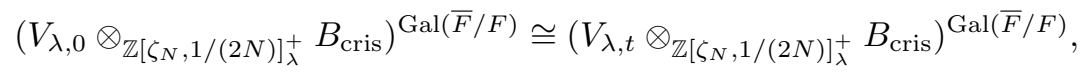

as both can be calculated from the crystalline cohomology of the base change of $Y_{0}$ to the residue field of $\mathcal{O}_{F}$. Thus, by Lemma $2.2, V_{\lambda, 0}$ is ordinary if and only if $V_{\lambda, t}$ is ordinary.) However if $\lambda^{\prime}$ is a prime of $\mathbb{Q}\left(\overline{\zeta_{N}}\right)$ above $\lambda$ then

$$
\begin{aligned}
V_{\lambda}((N-1-n) / 2)_{0} & \otimes_{\mathbb{Z}\left[\zeta_{N}, 1 /(2 N)\right]_{\lambda}^{+}} \overline{\mathbb{Q}}_{l} \\
& \cong \bigoplus_{i=(N+1-n) / 2}^{(N-1+n) / 2} U_{i, \lambda^{\prime}}((N-1-n) / 2) \otimes_{\mathbb{Z}\left[\zeta_{N}, 1 /(2 N)\right]_{\lambda^{\prime}}} \overline{\mathbb{Q}}_{l},
\end{aligned}
$$

and each $U_{i, \lambda^{\prime}}((N-1-n) / 2) \otimes_{\left.\mathbb{Z}\left[\zeta_{N}, 1 /(2 N)\right)\right]_{\lambda^{\prime}}} \overline{\mathbb{Q}}_{l}$ is crystalline. Suppose that $v^{\prime}$ is a place of $\mathbb{Q}\left(\zeta_{N}\right)$ above $v$; that $\sigma \in \operatorname{Gal}\left(\mathbb{Q}\left(\zeta_{N}\right) / \mathbb{Q}\right)$ is such that $\lambda^{\prime}$ corresponds to $v^{\prime} \circ \sigma^{-1}$; and that $\sigma^{-1}\left(\zeta_{N}\right)=\zeta_{N}^{a}$. Then the Hodge-Tate number of $U_{i, \lambda^{\prime}}((N-1-$ $n) / 2) \otimes_{\mathbb{Z}\left[\zeta_{N}, 1 /(2 N)\right]_{\lambda^{\prime}}} \overline{\mathbb{Q}}_{l}$ as a module for $\operatorname{Gal}\left({\overline{\mathbb{Q}}\left(\zeta_{N}\right)_{v^{\prime}}} / \mathbb{Q}\left(\zeta_{N}\right)_{v^{\prime}}\right)$ is

$$
\#\{(N+1-n) / 2 \leq b \leq(N-1+n) / 2: \overline{a b} \leq \overline{a i}\}-1 .
$$


Thus

$$
\begin{aligned}
U_{i, \lambda^{\prime}}((N-1-n) / 2) \otimes_{\mathbb{Z}\left[\zeta_{N}, 1 /(2 N)\right]_{\lambda^{\prime}}} \overline{\mathbb{Q}}_{l} \\
\quad \cong \overline{\mathbb{Q}}_{l}(1-\#\{(N+1-n) / 2 \leq b \leq(N-1+n) / 2: \overline{a b} \leq \overline{a i}\})
\end{aligned}
$$

as a module for $I_{\mathbb{Q}\left(\zeta_{N}\right)_{v}}$. The third and fourth parts follow. (Note that $l>N>n$, so that none of the characters $1, \epsilon_{l}^{-1}, \ldots, \epsilon_{l}^{1-n}$ of $I_{\mathbb{Q}\left(\zeta_{N}\right)_{v^{\prime}}}$ become congruent modulo $l$.)

For the fifth part it suffices to show that

$$
\begin{aligned}
& V_{\lambda}((N-1-n) / 2)_{0} \otimes_{\mathbb{Z}\left[\zeta_{N}, 1 /(2 N)\right]_{\lambda}^{+}} \overline{\mathbb{Q}}_{l} \\
& \cong\left(\overline{\mathbb{Q}}_{l}\left(\left(\epsilon_{l}^{(2)}\right)^{1-n}\right) \oplus \overline{\mathbb{Q}}_{l}\left(\left(\epsilon_{l}^{(2)}\right)^{2-n}\left(\epsilon_{l}^{(2), \text { Frob }_{l}}\right)^{-1}\right) \oplus \cdots \oplus \overline{\mathbb{Q}}_{l}\left(\left(\epsilon_{l}^{(2), \text { Frob }_{l}}\right)^{1-n}\right)\right)
\end{aligned}
$$

as a module for $I_{\mathbb{Q}\left(\zeta_{N}\right)_{v}^{+}}$. (Again note that none of these $n$ characters of $I_{\mathbb{Q}\left(\zeta_{N}\right)_{v}^{+}}$are congruent modulo $l$, because $(n-1)(l-1)<l^{2}-1$. Also recall the definition of the character $\epsilon_{l}^{(2)}$ of $\operatorname{Gal}\left(\overline{\mathbb{Q}}_{l} / \mathbb{Q}_{l^{2}}\right)$ given in the notation section after the introduction.) However

$$
\begin{aligned}
V_{\lambda}((N-1-n) / 2)_{0} & \otimes_{\mathbb{Z}\left[\zeta_{N}, 1 /(2 N)\right]_{\lambda}^{+}} \overline{\mathbb{Q}}_{l} \\
& \cong \bigoplus_{i=(N+1-n) / 2}^{(N-1+n) / 2} U_{i, \lambda}((N-1-n) / 2) \otimes_{\mathbb{Z}\left[\zeta_{N}, 1 /(2 N)\right]_{\lambda}} \overline{\mathbb{Q}}_{l}
\end{aligned}
$$

and each $U_{i, \lambda}((N-1-n) / 2) \otimes_{\left.\mathbb{Z}\left[\zeta_{N}, 1 /(2 N)\right)\right]_{\lambda}} \overline{\mathbb{Q}}_{l}$ is crystalline. Suppose $\sigma \in$ $\operatorname{Gal}\left(\mathbb{Q}\left(\zeta_{N}\right) / \mathbb{Q}\right)$ and $\lambda$ corresponds to $v \circ \sigma^{-1}$ and $\sigma^{-1}\left(\zeta_{N}\right)=\zeta_{N}^{a}$. There are two continuous embeddings $\mathbb{Q}\left(\zeta_{N}\right)_{v}$ into $\overline{\mathbb{Q}\left(\zeta_{N}\right)_{\lambda}}$, one being the continuous extension of $\sigma$ and the other $c \sigma=\sigma$ Frob $_{l}$. Then the Hodge-Tate number of $U_{i, \lambda}((N-1-n) / 2) \otimes_{\mathbb{Z}\left[\zeta_{N}, 1 /(2 N)\right]_{\lambda}} \overline{\mathbb{Q}}_{l}$ with respect to $\sigma$ (resp. $\sigma$ Frob $_{l}$ ) as a module for $\operatorname{Gal}\left(\overline{\mathbb{Q}\left(\zeta_{N}\right)_{v}} / \mathbb{Q}\left(\zeta_{N}\right)_{v}\right)$ is

$$
\#\{(N+1-n) / 2 \leq b \leq(N-1+n) / 2: \overline{a b} \leq \overline{a i}\}-1
$$

(resp.

$$
\begin{aligned}
\#\{(N+1-n) / 2 & \leq b \leq(N-1+n) / 2: \overline{-a b} \leq \overline{-a i}\}-1 \\
& =n-\#\{(N+1-n) / 2 \leq b \leq(N-1+n) / 2: \overline{a b} \leq \overline{a i}\}) .
\end{aligned}
$$

Thus

$$
U_{i, \lambda}((N-1-n) / 2) \otimes_{\mathbb{Z}\left[\zeta_{N}, 1 /(2 N)\right]_{\lambda}} \overline{\mathbb{Q}}_{l} \cong \overline{\mathbb{Q}}_{l}\left(\left(\sigma \epsilon_{l}^{(2)}\right)^{1-j}\left(\sigma \operatorname{Frob}_{l} \epsilon_{l}^{(2)}\right)^{j-n}\right)
$$

as a module for $I_{\mathbb{Q}\left(\zeta_{N}\right)_{v}}$, where

$$
j=\#\{(N+1-n) / 2 \leq b \leq(N-1+n) / 2: \overline{a b} \leq \overline{a i}\} .
$$




\section{$\S 6$. Potential modularity $\mathbf{I}$}

The arguments of this section follow those of [HSBT]. However we will make use of the improvements to [HT], CHT] and [Tay which have been made in [Sh], $\mathrm{CH}$, $\mathrm{Gu}$ and Ger] (which we have recalled in Sections 1 and 2 in order to in turn improve upon the results of [HSBT] (and also simplify some of the arguments in [HSBT).

We start with a result from elementary number theory.

Lemma 6.1. Suppose that $m$ is a positive integer, $l$ is a rational prime and $S$ is a finite set of rational primes. Then we can find a positive integer $N$ not divisible by any prime in $S$ such that if $l^{s} \equiv 1 \bmod N$ then $m \mid s$.

Proof. We may assume that $m=p^{t}$ is a prime power. Moreover we may assume that $t>1$ and that for each prime $q \in S \cup\{p\}$, either $q$ divides none of the numbers $l^{p^{u}}-1$ (for $u \in \mathbb{Z}_{\geq 0}$ ) or $q \mid\left(l^{p^{t-2}}-1\right.$ ). If a prime $r$ divides $l^{p^{t-1}}-1$ and $l^{p^{t-1}(p-1)}+\cdots+l^{p^{t-1}}+1$ then $r=p$. Moreover in that case

$$
l^{p^{t-1}(p-1)}+\cdots+l^{p^{t-1}}+1 \equiv p \bmod p^{2} .
$$

We conclude that it suffices to take $N$ to be any prime divisor of

$$
\left(l^{p^{t-1}(p-1)}+\cdots+l^{p^{t-1}}+1\right) /\left(p, l^{p^{t-1}(p-1)}+\cdots+l^{p^{t-1}}+1\right) .
$$

We will need yet another very minor variant of a theorem of Moret-Bailly MB] (see also GPR]).

Proposition 6.2. Let $F$ be a number field and let $S=S_{1} \amalg S_{2} \amalg S_{3}$ be a finite set of places of $F$, so that every element of $S_{2}$ is non-archimedean. Suppose that $M / F$ is a finite Galois extension such that every element of $S_{1}$ splits in $M$ and every element of $S_{2}$ is unramified in $S_{2}$. Let $S_{i}^{M}$ denote the set of places of $M$ above $S_{i}$. Suppose that $T / M$ is a smooth, geometrically connected variety. Suppose also that

- for $v \in S_{1}^{M}, \Omega_{v} \subset T\left(M_{v}\right)$ is a non-empty open subset (for the v-topology);

- for $v \in S_{2}^{M}, \Omega_{v} \subset T\left(M_{v}^{\mathrm{nr}}\right)$ is a non-empty open $\operatorname{Gal}\left(F_{v}^{\mathrm{nr}} / M_{v}\right)$-invariant subset;

- for $v \in S_{3}^{M}, \Omega_{v} \subset T\left(\bar{F}_{v}\right)$ is a non-empty open $\operatorname{Gal}\left(\bar{F}_{v} / M_{v}\right)$-invariant subset.

Suppose finally that $L / F$ is a finite Galois extension linearly disjoint from $M$. Then there is a finite Galois extension $F^{\prime} / F$ and a point $P \in T\left(F^{\prime}\right)$ such that

- $F^{\prime} \supset M$;

- $F^{\prime} / F$ is linearly disjoint from $L / F$;

- every place $v$ of $S_{1}$ splits completely in $F^{\prime}$ and if $w$ is a prime of $F^{\prime}$ above $v$ then $P \in \Omega_{\left.w\right|_{M}} \subset T\left(F_{w}^{\prime}\right)$; 
- every place $v$ of $S_{2}$ is unramified in $F^{\prime}$ and if $w$ is a prime of $F^{\prime}$ above $v$ then $P \in \Omega_{\left.w\right|_{M}} \cap T\left(F_{w}^{\prime}\right)$;

- if $w$ is a prime of $F^{\prime}$ above an element of $v \in S_{3}$ then $P \in \Omega_{\left.w\right|_{M}} \cap T\left(F_{w}^{\prime}\right)$.

Proof. Let $L_{1}, \ldots, L_{r}$ denote the intermediate fields $L \supset L_{i} \supset F$ with $L_{i} / F$ Galois with simple Galois group. Combining Hensel's lemma with the Weil bounds we see that $T$ has an $F_{v}$-rational point for all but finitely many primes $v$ of $F$. Thus enlarging $S_{1}$ to include, for each $i$, one sufficiently large prime that is split completely in $M$ but not split in $L_{i}$ (the prime may depend on $i$ ), we may suppress the second condition on $F^{\prime}$.

Let $M^{\prime} / F$ be a finite Galois extension such that

- $M^{\prime} \supset M$

- all places in $S_{1}$ split completely in $M^{\prime}$;

- every place $v$ of $S_{2}$ is unramified in $M^{\prime}$ and if $w$ is a prime of $M^{\prime}$ above $v$ then $\Omega_{\left.w\right|_{M}} \cap T\left(M_{w}^{\prime}\right) \neq \emptyset$

- if $w$ is a prime of $M^{\prime}$ above an element of $v \in S_{3}$ then $\Omega_{\left.w\right|_{M}} \cap T\left(M_{w}^{\prime}\right) \neq \emptyset$.

Theorem 1.3 of $\left[\mathrm{MB}\right.$ tells us that we can find a finite Galois extension $F^{\prime \prime} / M^{\prime}$ and a point $P \in T\left(F^{\prime \prime}\right)$ such that

- every place $v$ of $S_{1}$ splits completely in $F^{\prime \prime}$ and if $w$ is a prime of $F^{\prime \prime}$ above $v$ then $P \in \Omega_{v} \subset T\left(F_{w}^{\prime \prime}\right)$

- every place $v$ of $S_{2}$ is unramified in $F^{\prime \prime}$ and if $w$ is a prime of $F^{\prime \prime}$ above $v$ then $P \in \Omega_{v} \cap T\left(F_{w}^{\prime \prime}\right)$;

- if $w$ is a prime of $F^{\prime \prime}$ above an element of $v \in S_{3}$ then $P \in \Omega_{v} \cap T\left(F_{w}^{\prime \prime}\right)$.

Now take $F^{\prime}$ to be the normal closure of $F^{\prime \prime}$ over $F$.

We now turn to our first potential modularity theorem in the ordinary case.

Theorem 6.3. Suppose that $F$ is a totally real field and that $n$ is an even positive integer. Suppose that $l>n$ is a rational prime and that $L / \mathbb{Q}_{l}$ is a finite extension with ring of integers $\mathcal{O}$ and residue field $\mathbb{F}$. Suppose also that

$$
r: \operatorname{Gal}(\bar{F} / F) \rightarrow G S p_{n}(\mathcal{O})
$$

is a continuous representation which is unramified at all but finitely many primes. Let $\bar{r}$ denote the semisimplification of the reduction of $r$ modulo the maximal ideal of $\mathcal{O}$. Suppose moreover that $r$ enjoys the following properties:

1. $\bar{r}$ has multiplier $\epsilon_{l}^{1-n}$. 
2. The image $\bar{r}\left(\operatorname{Gal}\left(\bar{F} / F\left(\zeta_{l}\right)\right)\right)$ is big $\left(\right.$ in $\left.G L_{n}\left(\overline{\mathbb{F}}_{l}\right)\right)$ and $\zeta_{l} \notin \bar{F}^{\operatorname{ker} a d} \bar{r}$.

3. $r$ is ordinary of weight a for some $a \in\left(\mathbb{Z}^{n}\right)^{\operatorname{Hom}\left(F, \overline{\mathbb{Q}}_{l}\right),+}$.

Then there is a Galois totally real extension $F^{\prime} / F$ such that $\left.r\right|_{\mathrm{Gal}\left(\bar{F} / F^{\prime}\right)}$ is automorphic of weight $a_{F^{\prime}}$.

Proof. The strategy is to find an $N$ (as at the start of Section 4) and primes $\lambda, \lambda^{\prime}$ of $\mathbb{Q}\left(\zeta_{N}\right)^{+}$(with say $\left.\lambda^{\prime} \mid l^{\prime}\right)$ and $t \in T_{0}\left(F^{\prime}\right)$ such that $\left.V[\lambda]_{t} \cong \bar{r}\right|_{\operatorname{Gal}\left(\bar{F} / F^{\prime}\right)}$ while $\left.V\left[\lambda^{\prime}\right]_{t} \cong \bar{r}^{\prime}\right|_{\operatorname{Gal}\left(\bar{F} / F^{\prime}\right)}$, where $r^{\prime}: \operatorname{Gal}(\bar{F} / F) \rightarrow G L_{n}\left(\mathbb{Z}_{l^{\prime}}\right)$ is an ordinary weight 0 representation which is induced from a character $\theta$ of $\operatorname{Gal}(\bar{F} / F M)$ for a suitable CM field $M$. We will first choose $N$. Then we will choose $M$ and a character $\phi$ of $\mathbb{A}_{M}^{\times}$valued in a number field $M^{\prime} \supset M$. Then we choose $l^{\prime}$ to be a prime that splits in $M^{\prime}$ and take $\theta$ to be the $l^{\prime}$-adic character associated to $\phi$. Having made these choices, the bulk of the proof will be spent checking that $\bar{r}^{\prime}$ satisfies the conditions of Theorem 2.3. To enable us to check (inter alia) that $\bar{r}^{\prime} \operatorname{Gal}(\bar{F} / F)$ is big we arrange that $\phi$, and hence $\theta$, ramifies in a particular way above an auxiliary prime $q$. We then argue that as $r^{\prime}$ is automorphic over $F^{\prime}$, so is $\bar{r}^{\prime} \cong V\left[\lambda^{\prime}\right]_{t}$, and hence by Theorem 2.3 so is $V_{\lambda^{\prime}, t}$. Then $\bar{r} \cong V[\lambda]_{t}$ is automorphic over $F^{\prime}$ and hence, by Theorem 2.3 again, so is $r$.

Step 1: Choosing N, $\lambda, M, q, \phi, l^{\prime}, \lambda^{\prime}, \theta$ and $r^{\prime}$. Choose an odd positive integer $N$ divisible only by primes which do not ramify in $\bar{F}^{\text {ker }} \overline{(}\left(\zeta_{l}\right)$ and a prime $\lambda \mid l$ of $\mathbb{Q}\left(\zeta_{N}\right)^{+}$such that there is an embedding $\mathbb{F} \hookrightarrow \mathbb{Z}\left[\zeta_{N}\right]^{+} / \lambda$. (Use Lemma 6.1.) Fix such an embedding. Note that $\mathbb{Q}\left(\zeta_{N}\right)$ is linearly disjoint from $\bar{F}^{\operatorname{ker} \bar{r}}\left(\zeta_{l}\right)$ over $\mathbb{Q}$.

Choose an imaginary CM field $M$ which is cyclic Galois over $\mathbb{Q}$ of degree $n$ in which all primes which ramify in $\bar{F}^{\text {ker }} \bar{r}\left(\zeta_{N l}\right)$ are unramified. Let $\tau$ denote a generator of $\operatorname{Gal}(M / \mathbb{Q})$. Choose an odd rational prime $q \nmid N l$ which splits completely in $M$ and is unramified in $F$, and a prime $\mathfrak{q}$ of $M$ above $q$. Choose a finite extension $M^{\prime} / M$ and a character with open kernel

$$
\phi: \mathbb{A}_{M}^{\times} \rightarrow\left(M^{\prime}\right)^{\times}
$$

such that

- if $\alpha \in M^{\times}$then

$$
\phi(\alpha)=\prod_{i=0}^{n / 2-1} \tau^{i}(\alpha)^{i} \tau^{i}(c \alpha)^{n-1-i} ;
$$

- $\phi(\phi \circ c)=\prod_{v \nmid \infty}|\cdot|_{v}^{1-n}$;

- $\phi$ is unramified above $l N$;

- $\phi$ is unramified at all primes above $q$ except $\mathfrak{q}$ and $\mathfrak{q}^{c}$, but $q \mid \# \phi\left(\mathcal{O}_{M, \mathfrak{q}}^{\times}\right)$. 
(See Lemma 2.2 of [HSBT. .) Then choose a rational prime $l^{\prime}$ such that

- $l^{\prime}$ splits completely in $M^{\prime}\left(\zeta_{N}\right)$;

- $l^{\prime}$ is unramified in $F$;

- $\phi$ is unramified at $l^{\prime}$;

- $l^{\prime} \nmid l q$;

- $l^{\prime}>n+1$.

Also choose a prime $\lambda_{1} \mid l^{\prime}$ of $M^{\prime}$ and a prime $\lambda^{\prime} \mid l^{\prime}$ of $\mathbb{Q}\left(\zeta_{N}\right)^{+}$. Let

$$
\theta: \operatorname{Gal}(\overline{\mathbb{Q}} / \mathbb{Q}) \rightarrow \mathcal{O}_{M^{\prime}, \lambda_{1}}^{\times}=\mathbb{Z}_{l^{\prime}}^{\times}
$$

be the character defined by

$$
\theta(\operatorname{Art} \alpha)=\phi(\alpha) \prod_{i=0}^{n / 2-1} \tau^{i}\left(\alpha_{l^{\prime}}\right)^{-i}\left(\tau^{i} c\right)\left(\alpha_{l^{\prime}}\right)^{i+1-n},
$$

and let $\bar{\theta}$ denote the reduction of $\theta$ modulo $\lambda_{1}$. Note that $\theta \theta^{c}=\epsilon_{l^{\prime}}^{1-n}$.

Looking at inertia above $q$ we see that if $i, j, k \in \mathbb{Z} / n \mathbb{Z}$ and if

$$
\overline{\theta \theta}^{\tau^{k}}=\bar{\theta}^{\tau^{i}} \bar{\theta}^{\tau^{j}}
$$

on $\operatorname{Gal}\left(\overline{\mathbb{Q}} / F M\left(\zeta_{l^{\prime}}\right)\right)$ then we have one of the following alternatives ( $\left.\ddagger\right)$ : either

- $\{0, k\}=\{i, j\} \subset \mathbb{Z} / n \mathbb{Z}$, or

- $k=i-j=n / 2 \in \mathbb{Z} / n \mathbb{Z}$.

(Recall that $q \mid \# \bar{\theta}\left(I_{M_{\mathrm{q}}}\right)$ but that $\bar{\theta}$ is unramified at all other primes of $M$ above $q$ except $c \mathfrak{q}$. Note also that $\overline{\theta \theta}^{c}$ is unramified above q.)

Let $\widetilde{\tau} \in \operatorname{Gal}\left(\bar{F} / F\left(\zeta_{l^{\prime}}\right)\right)$ lift $\tau \in \operatorname{Gal}(M / \mathbb{Q})$. Then

$$
\bar{\theta}\left(\widetilde{\tau}^{n}\right)=\bar{\theta}\left(c\left(c \widetilde{\tau}^{n / 2}\right) c\left(c \widetilde{\tau}^{n / 2}\right)\right)=\left(\overline{\theta \theta}^{c}\right)\left(c \widetilde{\tau}^{n / 2}\right)=-1 .
$$

Also let $r^{\prime}$ denote the induction of $\theta$ from $\operatorname{Gal}(\overline{\mathbb{Q}} / M)$ to $\operatorname{Gal}(\overline{\mathbb{Q}} / \mathbb{Q})$ and let $\bar{r}^{\prime}$ denote the reduction of $r^{\prime}$ modulo $\lambda_{1}$. The representation $r^{\prime}$ has a basis $e_{0}, \ldots, e_{n-1}$ where

$$
\begin{array}{ll}
r^{\prime}(\sigma) e_{i}=\theta^{\tau^{i}}(\sigma) e_{i} & \text { for } \sigma \in \operatorname{Gal}(\bar{F} / M), \\
r^{\prime}(\widetilde{\tau}) e_{i}=e_{i-1} & \text { for } i=1, \ldots, n-1, \\
r^{\prime}(\widetilde{\tau}) e_{0}=-e_{n-1} . &
\end{array}
$$

Define a perfect alternating pairing on $r^{\prime}$ by

$$
\left\langle e_{i}, e_{j}\right\rangle= \begin{cases}1 & \text { if } j=i+n / 2 \\ -1 & \text { if } i=j+n / 2 \\ 0 & \text { otherwise }\end{cases}
$$


This pairing is preserved by $r$ up to scalar multiples and we see that

$$
r^{\prime}: \operatorname{Gal}(\bar{F} / \mathbb{Q}) \rightarrow G S p_{n}\left(\mathbb{Z}_{l^{\prime}}\right)
$$

with multiplier $\epsilon_{l^{\prime}}^{1-n}$.

Note that $\bar{F}^{\operatorname{ker}\left(\bar{r} \times \bar{r}^{\prime}\right)}\left(\zeta_{l l^{\prime}}\right)$ is linearly disjoint from $\mathbb{Q}\left(\zeta_{N}\right)$ over $\mathbb{Q}$ (as they ramify at disjoint sets of primes).

Step 2: Checking the conditions of Theorem 2.3 for $\bar{r}^{\prime}$. Let $f_{0}, \ldots, f_{n-1}$ denote the basis of $\operatorname{Hom}\left(r^{\prime}, \mathbb{Z}_{l^{\prime}}\right)$ dual to $e_{0}, \ldots, e_{n-1}$, so that $\left\{e_{i} \otimes f_{j}\right\}$ is a basis of ad $r^{\prime}$. Then we can decompose

$$
\operatorname{ad} r^{\prime} \otimes_{\mathbb{Z}_{l^{\prime}}} \overline{\mathbb{F}}_{l^{\prime}}=\left(\bigoplus_{\chi \in \operatorname{Hom}\left(\operatorname{Gal}(M / \mathbb{Q}), \overline{\mathbb{F}}_{l^{\prime}}\right)} W_{\chi}\right) \oplus\left(\bigoplus_{i=1}^{n-1} W_{i}\right),
$$

where

- $W_{\chi}$ is the span of $\sum_{i=0}^{n-1} \chi\left(\tau^{i}\right) e_{i} \otimes f_{i}$;

- $W_{i}$ is the span of $\left\{e_{j} \otimes f_{i+j}\right\}_{j=0, \ldots, n-1}$.

Thus $W_{\chi} \cong \overline{\mathbb{F}}_{l^{\prime}}(\chi)$ and

$$
W_{i} \cong \operatorname{Ind}_{\operatorname{Gal}(\bar{F} / M)}^{\operatorname{Gal}(\bar{Q})} \bar{\theta} / \bar{\theta}^{i}
$$

For $i, j=1, \ldots, n-1$ we have $\bar{\theta} / \bar{\theta}^{\tau^{i}} \neq \bar{\theta}^{\tau^{j}} / \bar{\theta}^{\tau^{i+j}}$ on $\operatorname{Gal}\left(\bar{F} / F M\left(\zeta_{l^{\prime}}\right)\right)$ (see alternative $(\ddagger)$ above) and so we deduce that $W_{i}$ is an irreducible $\overline{\mathbb{F}} l^{\prime}\left[\operatorname{Gal}\left(\bar{F} / F\left(\zeta_{l^{\prime}}\right)\right]\right.$ module. If $i, i^{\prime}=1, \ldots, n-1, j=0, \ldots, n-1$ and $\bar{\theta} / \bar{\theta}^{\tau^{i}}=\bar{\theta}^{\tau^{j}} / \bar{\theta}^{\tau^{i^{\prime}+j}}$ on $\operatorname{Gal}\left(\bar{F} / F M\left(\zeta_{l^{\prime}}\right)\right)$ then we see that either $i=i^{\prime}$ or $i+i^{\prime}=n$ (see alternative ( ) above). Thus $W_{i} \cong W_{i^{\prime}}$ as $\operatorname{Gal}\left(\bar{F} / F\left(\zeta_{l^{\prime}}\right)\right)$-modules (if and) only if $i=i^{\prime}$ or $i+i^{\prime}=n$.

We can choose $\sigma_{0} \in \operatorname{Gal}\left(\overline{\mathbb{Q}} / M\left(\zeta_{l^{\prime}}\right)\right)$ such that $\bar{\theta}\left(\sigma_{0}\right)$ has order divisible by $q$ but $\bar{\theta}^{\tau^{i}}\left(\sigma_{0}\right)=1$ for $i=1, \ldots, n / 2-1$. (For instance choose $\sigma_{0}$ in the inertia group at $\mathfrak{q}$ and recall that $q \mid \# \bar{\theta}\left(I_{M_{\mathfrak{q}}}\right)$.) Then

$$
\pi_{\bar{r}^{\prime}\left(\sigma_{0}\right), \bar{\theta}\left(\sigma_{0}\right)}\left(\sum_{i=0}^{n-1} \chi\left(\tau^{i}\right) e_{i} \otimes f_{i}\right) i_{\bar{r}^{\prime}\left(\sigma_{0}\right), \bar{\theta}\left(\sigma_{0}\right)}=1
$$

and so

$$
\pi_{\bar{r}^{\prime}\left(\sigma_{0}\right), \bar{\theta}\left(\sigma_{0}\right)} W_{\chi} i_{\bar{r}^{\prime}\left(\sigma_{0}\right), \bar{\theta}\left(\sigma_{0}\right)} \neq(0)
$$

for all $\chi \in \operatorname{Hom}\left(\operatorname{Gal}(M / \mathbb{Q}), \overline{\mathbb{F}}_{l^{\prime}}^{\times}\right)$. If $W$ is any other irreducible $\operatorname{Gal}\left(\bar{F} / F\left(\zeta_{l^{\prime}}\right)\right.$ submodule of ad $r \otimes \overline{\mathbb{F}}_{l^{\prime}}$ then it contains a vector either of the form

$$
\alpha e_{0} \otimes f_{i}+\beta e_{n / 2+i} \otimes f_{n / 2}
$$


for some $i=1, \ldots, n / 2-1$, or of the form

$$
e_{0} \otimes f_{n / 2} .
$$

Let $\zeta$ denote a non-trivial $n^{\text {th }}$ root of unity in $\overline{\mathbb{F}}_{l^{\prime}}$ with, in the former case, $\alpha+$ $\zeta^{-2 i} \beta \neq 0$. Then either

$$
\pi_{\bar{r}^{\prime}(\widetilde{\tau}), \zeta}\left(\alpha e_{0} \otimes f_{i}+\beta e_{n / 2+i} \otimes f_{n / 2}\right) i_{\bar{r}^{\prime}(\widetilde{\tau}), \zeta}=\zeta^{i}\left(\alpha+\zeta^{-2 i} \beta\right) / n \neq 0,
$$

or

$$
\pi_{\bar{r}^{\prime}(\widetilde{\tau}), \zeta}\left(e_{0} \otimes f_{n / 2}\right) i_{\bar{r}^{\prime}(\widetilde{\tau}), \zeta}=\zeta^{n / 2} / n .
$$

As $l$ does not divide $\# \bar{r}^{\prime}\left(\operatorname{Gal}\left(\bar{F} / F\left(\zeta_{l^{\prime}}\right)\right)\right)$, we conclude that $\bar{r}^{\prime}\left(\operatorname{Gal}\left(\bar{F} / F\left(\zeta_{l^{\prime}}\right)\right)\right)$ is big.

Choose $\sigma_{1} \in \operatorname{Gal}(\bar{F} / F M)$ such that $\epsilon_{l^{\prime}}\left(\sigma_{1}\right) \bmod l^{\prime}$ has order $l^{\prime}-1$ (e.g. choose a suitable element of an inertia group above $\left.l^{\prime}\right)$. Let

$$
\sigma_{2}=\prod_{j=0}^{n-1} \widetilde{\tau}^{j} \sigma_{1} \widetilde{\tau}^{-j}
$$

Then

$$
\bar{\theta}^{\tau^{i}}\left(\sigma_{2}\right)=\prod_{j=0}^{n-1} \bar{\theta}^{\tau^{j}}\left(\sigma_{1}\right)
$$

is independent of $i$ so that ad $\bar{r}^{\prime}\left(\sigma_{2}\right)=1$. However

$$
\epsilon_{l^{\prime}}\left(\sigma_{2}\right) \bmod l^{\prime}=\epsilon_{l^{\prime}}\left(\sigma_{1}\right)^{n} \bmod l^{\prime} \neq 1 .
$$

Thus $\left.\zeta_{l^{\prime}} \notin \bar{F}^{(\operatorname{ker} a d} \bar{r}^{\prime}\right) \cap \operatorname{Gal}(\bar{F} / F)$.

Step 3: Completion of the proof. Let $T_{0}$ be the scheme described in Section 4 corresponding to $N$ and let $V_{\lambda}$ and $V_{\lambda^{\prime}}$ be the sheaves on $\left(T_{0} \times \operatorname{Spec} F\right)_{\text {et }}$ corresponding to $N$ and $n$. We can choose a Galois extension $F^{\prime} / F$ and a point $t \in T_{0}\left(F^{\prime}\right)$ such that

- $F^{\prime} \supset F\left(\zeta_{N}\right)^{+}$

- $F^{\prime}$ is totally real;

- $F^{\prime} / F$ is linearly disjoint from $\bar{F}^{\operatorname{ker}\left(\bar{r} \times \bar{r}^{\prime}\right)}\left(\zeta_{l l^{\prime}}\right)$ over $F$;

- $\left.V[\lambda]_{t} \cong \bar{r}\right|_{\operatorname{Gal}\left(\bar{F} / F^{\prime}\right)}$;

- $\left.V\left[\lambda^{\prime}\right]_{t} \cong \bar{r}^{\prime}\right|_{\operatorname{Gal}\left(\bar{F} / F^{\prime}\right)}$

- $v(t)<0$ for all places $v \mid l$ of $F^{\prime}$

- $v(t)>0$ for all places $v \mid l^{\prime}$ of $F^{\prime}$. 
(Apply Moret-Bailly's Proposition 6.2 to $T_{\bar{r} \times \bar{r}^{\prime}}$ as in the proof of Theorem 3.1 of HSBT. We take $F$ of Proposition 6.2 to be $F$; we take $M$ of Proposition 6.2 to be $F\left(\zeta_{N}\right)^{+}$; and we take $L$ of Proposition 6.2 to be $\bar{F}^{\operatorname{ker}\left(\bar{r} \times \bar{r}^{\prime}\right)}\left(\zeta_{l l^{\prime}}\right)$. We take $T$ of Proposition 6.2 to be $T_{\bar{r} \times \bar{r}^{\prime}}$, as defined in the paragraph after the proof of Lemma 4.1. We also take $S_{1}$ to consist of the infinite places of $F$, and $S_{3}$ to consist of the divisors of $l l^{\prime}$ (and $S_{2}=\emptyset$ ). For $v \in S_{1}^{F\left(\zeta_{N}\right)^{+}}$we take $\Omega_{v}=T_{\bar{r} \times \bar{r}^{\prime}}\left(F\left(\zeta_{N}\right)_{v}^{+}\right)$. For $v \mid l$ we take $\Omega_{v}$ to be the set of points in $T_{\bar{r} \times \bar{r}^{\prime}}\left(\bar{F}_{v}\right)$ which map to a point $t \in T_{0}\left(\bar{F}_{v}\right) \subset \bar{F}_{v}$ with $v(t)<0$. For $v \mid l^{\prime}$ we take $\Omega_{v}$ to be the set of points in $T_{\bar{r} \times \bar{r}^{\prime}}\left(\bar{F}_{v}\right)$ which map to a point $t \in T_{0}\left(\bar{F}_{v}\right) \subset \bar{F}_{v}$ with $v(t)>0$. Note that by Proposition 4.2. $T_{\bar{r} \times \bar{r}^{\prime}}$ is geometrically connected. If $v \mid \infty$ then any point in $T_{0}\left(F_{v}\right)$ lifts to a point in $T_{\bar{r} \times \bar{r}^{\prime}}\left(F_{v}\right)$ as all involutions in $G S p_{n}(k)$ with multiplier -1 are conjugate (for any field $k$ of characteristic not 2). Hence for $v \mid \infty$ we have $\Omega_{v} \neq \emptyset$.)

From Theorem 4.2 of $[\mathrm{AC}]$ we know that $r^{\prime}$ is automorphic over $F^{\prime}$ of weight 0 . Thus $\bar{r}^{\prime} \cong V\left[\lambda^{\prime}\right]_{t}$ is ordinarily automorphic over $F^{\prime}$ of level prime to $l^{\prime}$. By Lemma 5.3 we see that $V_{\lambda^{\prime}, t}$ is ordinary of weight 0 . By Theorem 2.3 we see that it is automorphic of weight 0. Moreover it follows from Lemma 5.1 and Theorem 1.1 that it arises from a RAESDC automorphic representation $\pi$ of weight 0 with $\pi_{v}$ a Steinberg representation for all $v \mid l$. (Because $\pi_{v}$ is generic, $r_{l}\left(\imath^{-1} \pi_{v}\right)^{\vee}(1-n)^{\mathrm{ss}}$ is unramified and $r_{l}\left(\imath^{-1} \pi_{v}\right)^{\vee}(1-n)^{\mathrm{ss}}\left(\right.$ Frob $\left._{v}\right)$ has eigenvalues of the form $\left\{\alpha, \alpha(\# k(v)), \ldots, \alpha(\# k(v))^{n-1}\right\}$, it follows that $\pi_{v}$ is Steinberg.) Thus $\bar{r} \cong V_{t}[\lambda]$ is ordinarily Steinberg automorphic over $F^{\prime}$. Applying Theorem 2.3 again we conclude that $r$ is automorphic over $F^{\prime}$ of weight $a_{F^{\prime}}$.

We now turn to a first potential modularity theorem for the case of 'niveau two'.

Theorem 6.4. Suppose that $F$ is a totally real field and that $n$ is an even positive integer. Let $S$ denote a finite set of rational primes which contains 2 and all primes which ramify in $F$. Suppose that $l$ is a rational prime and write $l+1=N_{1} N_{2}$, where $N_{1}$ is divisible only by primes in $S$ and $N_{2}$ is divisible by no prime in $S$. Suppose that $N_{2}>n+1$. Suppose also that

$$
r: \operatorname{Gal}(\bar{F} / F) \rightarrow G S p_{n}\left(\mathbb{Z}_{l}\right)
$$

is a continuous representation which is unramified outside $S \cup\{l\}$. Let $\bar{r}$ denote the semisimplification of the reduction of $r$ modulo $l$. Suppose moreover that $r$ enjoys the following properties:

1. $\bar{r}$ has multiplier $\epsilon_{l}^{1-n}$.

2. The image $\bar{r}\left(\operatorname{Gal}\left(\bar{F} / F\left(\zeta_{l}\right)\right)\right)$ is big $\left(\right.$ in $\left.G L_{n}\left(\overline{\mathbb{F}}_{l}\right)\right)$ and $\zeta_{l} \notin \bar{F}^{\mathrm{kerad} \bar{r}}$. 
3. - $l$ is unramified in $F$;

- if $v \mid l$ is a prime of $F$ then $r$ is crystalline at $v$;

- if $v \mid l$ is a prime of $F$ then

$$
\left.\bar{r}\right|_{I_{F_{v}}} \sim \omega_{2}^{1-n} \oplus \omega_{2}^{-l+2-n} \oplus \omega_{2}^{-2 l+3-n} \oplus \cdots \oplus \omega_{2}^{(1-n) l} ;
$$

- if $v \mid l$ is a prime of $F$ then

$$
\operatorname{dim}_{F_{v}} \operatorname{gr}^{i}\left(r \otimes_{\mathbb{Z}_{l}} B_{\mathrm{DR}}\right)^{\operatorname{Gal}\left(\bar{F}_{v} / F_{v}\right)}=0
$$

unless $0 \leq i<n$, in which case

$$
\operatorname{dim}_{F_{v}} \operatorname{gr}^{i}\left(r \otimes_{\mathbb{Z}_{l}} B_{\mathrm{DR}}\right)^{\operatorname{Gal}\left(\bar{F}_{v} / F_{v}\right)}=1 .
$$

Then there is a Galois totally real extension $F^{\prime} / F$ such that $\left.r\right|_{\mathrm{Gal}\left(\bar{F} / F^{\prime}\right)}$ is automorphic of weight 0 .

Proof. The strategy is very similar to the proof of Theorem 6.3. We apply the results of Section 4 with $N=N_{2}$. We will find primes $\lambda, \lambda^{\prime}$ of $\mathbb{Q}\left(\zeta_{N_{2}}\right)^{+}$(with say $\left.\lambda^{\prime} \mid l^{\prime}\right)$ and $t \in T_{0}\left(F^{\prime}\right)$ such that $\left.V[\lambda]_{t} \cong \bar{r}\right|_{\operatorname{Gal}\left(\bar{F} / F^{\prime}\right)}$ while $\left.V\left[\lambda^{\prime}\right]_{t} \cong \bar{r}^{\prime}\right|_{\operatorname{Gal}\left(\bar{F} / F^{\prime}\right)}$, where $r^{\prime}: \operatorname{Gal}(\bar{F} / F) \rightarrow G L_{n}\left(\mathbb{Z}_{l^{\prime}}\right)$ is an ordinary weight 0 representation which is induced from a character $\theta$ of $\operatorname{Gal}(\bar{F} / F M)$ for a suitable $\mathrm{CM}$ field $M$. We first choose $M$ and a character $\phi$ of $\mathbb{A}_{M}^{\times}$valued in a number field $M^{\prime} \supset M$. Then we choose $l^{\prime}$ to be a prime that splits in $M^{\prime}$ and take $\theta$ to be the $l^{\prime}$-adic character associated to $\phi$. (If we choose $l^{\prime}$ first it is hard to construct a suitable $\theta$ valued in $\mathbb{Z}_{l^{\prime}}^{\times}$, as opposed to some extension of $\mathbb{Z}_{l^{\prime}}$.) Having made these choices we must check that $\bar{r}^{\prime}$ satisfies the conditions of Theorem 2.3. To enable us to check (inter alia) that $\bar{r}^{\prime}(\operatorname{Gal}(\bar{F} / F))$ is big we arrange that $\phi$ and hence $\theta$ ramifies in a particular way above an auxiliary prime $q$. We then argue that as $r^{\prime}$ is automorphic over $F^{\prime}$, so is $\bar{r}^{\prime} \cong V\left[\lambda^{\prime}\right]_{t}$, and hence by Theorem 2.3 so is $V_{\lambda^{\prime}, t}$. Then $\bar{r} \cong V[\lambda]_{t}$ is automorphic over $F^{\prime}$ and hence, by Theorem 2.1, so is $r$.

We remark that in order to apply Theorem 2.1 we need to choose $F^{\prime} / F$ unramified above $l$. (This is in contrast with the proof of Theorem 6.3. where we could apply the stronger Theorem 2.3.) To find such an $F^{\prime}$ we need some $t_{0}$ in the maximal unramified extension of $\mathbb{Q}_{l}$ such that

$$
V[\lambda]_{t_{0}} \sim \omega_{2}^{1-n} \oplus \omega_{2}^{-l+2-n} \oplus \omega_{2}^{-2 l+3-n} \oplus \cdots \oplus \omega_{2}^{(1-n) l}
$$

as a representation of $I_{\mathbb{Q}_{l}}$. The only suitable $t_{0}$ we know of is $t_{0}=0$ and this only works if $N \mid(l+1)$. This in particular implies that $l$ splits in $\mathbb{Q}\left(\zeta_{N}\right)^{+}$, so that $\lambda$ has residue field $\mathbb{F}_{l}$. Thus we need to assume that $\bar{r}$ is valued in $G S p_{n}\left(\mathbb{F}_{l}\right)$. (In fact for simplicity we assume that $r$ is valued in $G S p_{n}\left(\mathbb{Z}_{l}\right)$, but all the proof actually requires is that $\bar{r}$ is valued in $G S p_{n}\left(\mathbb{F}_{l}\right)$.) 
Step 1: Choosing $M, q, \phi, l^{\prime}, \theta$ and $r^{\prime}$. Choose an imaginary CM field $M$ which is cyclic Galois over $\mathbb{Q}$ of degree $n$ in which all primes which ramify in $\bar{F}^{\operatorname{ker} \bar{r}}\left(\zeta_{N_{2} l}\right)$ are unramified. Let $\tau$ denote a generator of $\operatorname{Gal}(M / \mathbb{Q})$. Choose an odd rational prime $q \nmid N_{2} l$ which splits completely in $M$ and is unramified in $F$, and a prime $\mathfrak{q}$ of $M$ above $q$. Choose a finite CM extension $M^{\prime} / M$ which is Galois over $\mathbb{Q}$, and a character with open kernel $\phi: \mathbb{A}_{M}^{\times} \rightarrow\left(M^{\prime}\right)^{\times}$such that

- if $\alpha \in M^{\times}$then

$$
\phi(\alpha)=\prod_{i=0}^{n / 2-1} \tau^{i}(\alpha)^{i} \tau^{i}(c \alpha)^{n-1-i}
$$

- $\phi(\phi \circ c)=\prod_{v \nmid \infty}|\cdot|_{v}^{1-n}$;

- $\phi$ is unramified above $l N_{2}$;

- $\phi$ is unramified at all primes above $q$ except $\mathfrak{q}$ and $\mathfrak{q}^{c}$, but $q \mid \# \phi\left(\mathcal{O}_{M, \mathfrak{q}}^{\times}\right)$.

(See Lemma 2.2 of [HSBT. .) Then choose a rational prime $l^{\prime}$ such that

- $l^{\prime}$ splits completely in $M^{\prime}\left(\zeta_{N_{2}}\right)$;

- $l^{\prime}$ unramified in $F\left(\zeta_{l q}\right)$;

- $\phi$ unramified at $l^{\prime}$;

- $l^{\prime}>n+1$

Also choose a prime $\lambda_{1} \mid l^{\prime}$ of $M^{\prime}$. Let

$$
\theta: \operatorname{Gal}(\overline{\mathbb{Q}} / \mathbb{Q}) \rightarrow \mathcal{O}_{M^{\prime}, \lambda_{1}}^{\times}=\mathbb{Z}_{l^{\prime}}^{\times}
$$

be the character defined by

$$
\theta(\operatorname{Art} \alpha)=\phi(\alpha) \prod_{i=0}^{n / 2-1} \tau^{i}\left(\alpha_{l^{\prime}}\right)^{-i}\left(\tau^{i} c\right)\left(\alpha_{l^{\prime}}\right)^{i+1-n},
$$

and let $\bar{\theta}$ denote the reduction of $\theta$ modulo $\lambda_{1}$. Also let $r^{\prime}$ denote the induction of $\theta$ from $\operatorname{Gal}(\overline{\mathbb{Q}} / M)$ to $\operatorname{Gal}(\overline{\mathbb{Q}} / \mathbb{Q})$ and let $\bar{r}^{\prime}$ denote the reduction of $r^{\prime}$ modulo $\lambda_{1}$. Exactly as in the proof of Theorem 6.3 we see that

$$
r^{\prime}: \operatorname{Gal}(\bar{F} / \mathbb{Q}) \rightarrow G S p_{n}\left(\mathbb{Z}_{l^{\prime}}\right),
$$

that $\bar{r}^{\prime}\left(\operatorname{Gal}\left(\bar{F} / F\left(\zeta_{l^{\prime}}\right)\right)\right)$ is big, and that $\left.\zeta_{l^{\prime}} \notin \bar{F}^{(\operatorname{ker} a d} \bar{r}^{\prime}\right) \cap \operatorname{Gal}(\bar{F} / F)$.

Step 2: Completion of the proof. Let $\lambda$ and $\lambda^{\prime}$ be primes of $\mathbb{Q}\left(\zeta_{N_{2}}\right)$ above $l$ and $l^{\prime}$. Let $T_{0}$ be the scheme described in Section 4 corresponding to $N_{2}$ and let $V_{\lambda}$ and $V_{\lambda^{\prime}}$ be the sheaves on $\left(T_{0} \times \operatorname{Spec} F\right)_{\text {et }}$ corresponding to $N_{2}$ and $n$. We can choose a Galois extension $F^{\prime} / F$ and a point $t \in T_{0}\left(F^{\prime}\right)$ such that

- $F^{\prime} \supset \mathbb{Q}\left(\zeta_{N_{2}}\right)^{+}$;

- $F^{\prime}$ is totally real and $l$ is unramified in $F^{\prime}$; 
- $F^{\prime} / F$ is linearly disjoint from $\bar{F}^{\operatorname{ker}\left(\bar{r} \times \bar{r}^{\prime}\right)}\left(\zeta_{l l^{\prime}}\right)$ over $F$;

- $\left.V[\lambda]_{t} \cong \bar{r}\right|_{\operatorname{Gal}\left(\bar{F} / F^{\prime}\right)}$

- $\left.V\left[\lambda^{\prime}\right]_{t} \cong \bar{r}^{\prime}\right|_{\operatorname{Gal}\left(\bar{F} / F^{\prime}\right)}$

- $v(t)>0$ for all places $v \mid l$ of $F^{\prime}$

- $v(t)>0$ for all places $v \mid l^{\prime}$ of $F^{\prime}$.

Apply Moret-Bailly's Proposition 6.2 to $T_{\bar{r} \times \bar{r}^{\prime}}$ as in the proof of Theorem 3.1 of HSBT. We also take $S_{1}$ to consist of the infinite places of $F$ and $S_{3}$ to consist of the divisors of $l l^{\prime}$ (and $S_{2}=\emptyset$ ). For $v \in S_{1}^{F\left(\zeta_{N}\right)^{+}}$we take $\Omega_{v}=T_{\bar{r} \times \bar{r}^{\prime}}\left(F\left(\zeta_{N}\right)_{v}^{+}\right)$. For $v \mid l$ we take $\Omega_{v}$ to be the set of points in $T_{\bar{r} \times \bar{r}^{\prime}}\left(\bar{F}_{v}\right)$ which map to a point $t \in T_{0}\left(\bar{F}_{v}\right) \subset \bar{F}_{v}$ with $v(t)<0$. For $v \mid l^{\prime}$ we take $\Omega_{v}$ to be the set of points in $T_{\bar{r} \times \bar{r}^{\prime}}\left(\bar{F}_{v}\right)$ which map to a point $t \in T_{0}\left(\bar{F}_{v}\right) \subset \bar{F}_{v}$ with $v(t)>0$. Note that by Proposition 4.2, $T_{\bar{r} \times \bar{r}^{\prime}}$ is geometrically connected. If $v \mid \infty$ then any point in $T_{0}\left(F_{v}\right)$ lifts to a point in $T_{\bar{r} \times \bar{r}^{\prime}}\left(F_{v}\right)$ as all involutions in $\operatorname{GSp}_{n}(k)$ with multiplier -1 are conjugate (for any field $k$ of characteristic not 2). Hence for $v \mid \infty$ we have $\Omega_{v} \neq \emptyset$. (Apply Moret-Bailly's Proposition 6.2 to $T_{\bar{r} \times \bar{r}^{\prime}}$ as in the proof of Theorem 3.1 of [HSBT]. We take $F$ of Proposition 6.2 to be $F$; we take $M$ of Proposition 6.2 to be $F\left(\zeta_{N_{2}}\right)^{+}$; and we take $L$ of Proposition 6.2 to be $\bar{F}^{\operatorname{ker}\left(\bar{r} \times \bar{r}^{\prime}\right)}\left(\zeta_{l l^{\prime}}\right)$. We take $T$ of Proposition 6.2 to be $T_{\bar{r} \times \bar{r}^{\prime}}$, as defined in the paragraph after the proof of Lemma 4.1. We take $S_{1}$ to consist of the infinite places of $F$ and $S_{2}$ to consist of places above $l$, and $S_{3}$ to consist of places above $l^{\prime}$. For $v \in S_{1}^{F\left(\zeta_{N_{2}}\right)^{+}}$we take $\Omega_{v}=T_{\bar{r} \times \bar{r}^{\prime}}\left(F\left(\zeta_{N_{2}}\right)_{v}^{+}\right)$, which is non-empty as in the proof of Theorem 6.3 . For $v \mid l$ we take $\Omega_{v}$ to be the set of points in $T_{\bar{r} \times \bar{r}^{\prime}}\left(F_{v}^{\text {nr }}\right)$ which map to a point $t \in T_{0}\left(F_{v}^{\mathrm{nr}}\right) \subset F_{v}^{\mathrm{nr}}$ with $v(t)>0$. This is non-empty as by Lemma 5.3 it contains a point above $0 \in T_{0}\left(F_{v}^{\mathrm{nr}}\right)$. For $v \mid l^{\prime}$ we take $\Omega_{v}$ to be the set of points in $T_{\bar{r} \times \bar{r}^{\prime}}\left(\bar{F}_{v}\right)$ which map to a point $t \in T_{0}\left(\bar{F}_{v}\right) \subset \bar{F}_{v}$ with $v(t)>0$. Note that by Proposition 4.2. $T_{\bar{r} \times \bar{r}^{\prime}}$ is geometrically connected.)

From Theorem 4.2 of $[\mathrm{AC}]$ we know that $r^{\prime}$ is automorphic over $F^{\prime}$ of weight 0 . Thus $\bar{r}^{\prime} \cong V\left[\lambda^{\prime}\right]_{t}$ is ordinarily automorphic over $F^{\prime}$ of level prime to $l^{\prime}$. By Lemma 5.3 we see that $V_{\lambda^{\prime}, t}$ is ordinary of weight 0 . By Theorem 2.3 we see that it is automorphic of weight 0 . Moreover by Lemma 5.1 and Theorem 1.1 , it arises from a RAESDC automorphic representation $\pi$ of weight 0 with $\pi_{v}$ unramified for all $v \mid l$. Thus $\bar{r} \cong V_{t}[\lambda]$ is automorphic over $F^{\prime}$ of level prime to $l$. Applying Theorem 2.1 we conclude that $r$ is automorphic over $F^{\prime}$ of weight 0 .

We remark that the condition on $l$ in the first paragraph of the theorem only excludes a set of primes of Dirichlet density zero. (This may be proved just the same way as Proposition 11 of [BL1.) 


\section{$\S 7$. Potential modularity II}

In this section we will use an idea of $[\mathrm{H}]$ to improve upon the theorems of the last section. Before turning to the main business of this section, let us recall some facts about infinitesimal characters.

Let $T \subset G L_{n}$ denote the diagonal maximal torus. Also let $\widetilde{T}$ denote the restriction of scalars of $T_{\mathbb{C}}$ from $\mathbb{C}$ to $\mathbb{R}$. We will identify

$$
X^{*}(T) \cong \mathbb{Z}^{n} \quad \text { via } \quad\left(m_{1}, \ldots m_{n}\right): \operatorname{diag}\left(t_{1}, \ldots, t_{n}\right) \mapsto t_{1}^{m_{1}} \ldots t_{n}^{m_{n}} .
$$

The Weyl group of $T$ in $G L_{n}$ is the symmetric group $S_{n}$ and under the above identification it acts on $\mathbb{Z}^{n}$ by permuting the coordinates. Similarly we may identify

$$
X^{*}\left(\widetilde{T} \times_{\mathbb{R}} \mathbb{C}\right) \cong X^{*}(T) \oplus X^{*}(T) \cong \mathbb{Z}^{n} \oplus \mathbb{Z}^{n},
$$

where

$$
\left(\left(m_{1}, \ldots, m_{n}\right),\left(m_{1}^{\prime}, \ldots, m_{n}^{\prime}\right)\right): \operatorname{diag}\left(t_{1}, \ldots, t_{n}\right) \mapsto t_{1}^{m_{1}}\left(c t_{1}\right)^{m_{1}^{\prime}} \ldots t_{n}^{m_{n}}\left(c t_{n}\right)^{m_{n}^{\prime}} .
$$

The Weyl group of $\widetilde{T} \times \mathbb{C}$ in the base change to $\mathbb{C}$ of the restriction of scalars of $G L_{n}$ from $\mathbb{C}$ to $\mathbb{R}$ is $S_{n} \times S_{n}$, which acts on $\mathbb{Z}^{n} \oplus \mathbb{Z}^{n}$ by each factor of $S_{n} \times S_{n}$ permuting the coordinates of the corresponding factor of $\mathbb{Z}^{n} \oplus \mathbb{Z}^{n}$.

The centre of the universal enveloping algebra of $\left(\right.$ Lie $\left.G L_{n}(\mathbb{R})\right) \otimes_{\mathbb{R}} \mathbb{C}$ (resp. $\left(\right.$ Lie $\left.G L_{n}(\mathbb{C})\right) \otimes_{\mathbb{R}} \mathbb{C}$ ) acts by a character (the infinitesimal character) on any irreducible admissible $\left(\left(\right.\right.$ Lie $\left.\left.G L_{n}(\mathbb{R})\right) \otimes_{\mathbb{R}} \mathbb{C}, O(n)\right)$-module $\left(\operatorname{resp} .\left(\left(\right.\right.\right.$ Lie $\left.G L_{n}(\mathbb{C})\right) \otimes_{\mathbb{R}} \mathbb{C}$, $U(n)$ )-module) $\pi$. Harish-Chandra parametrised such a character by an element

$$
z_{\pi} \in\left(X^{*}(T) \otimes_{\mathbb{Z}} \mathbb{C}\right) / S_{n} \cong \mathbb{C}^{n} / S_{n}
$$

(resp.

$$
\left.z_{\pi} \in\left(X^{*}\left(\widetilde{T} \times_{\mathbb{R}} \mathbb{C}\right) \otimes_{\mathbb{Z}} \mathbb{C}\right) /\left(S_{n} \times S_{n}\right) \cong \mathbb{C}^{n} / S_{n} \times \mathbb{C}^{n} / S_{n}\right)
$$

We call $\pi$ regular if the pre-image of $z_{\pi}$ in $X^{*}(T) \otimes_{\mathbb{Z}} \mathbb{C}\left(\right.$ resp. $\left.X^{*}\left(\widetilde{T} \times_{\mathbb{R}} \mathbb{C}\right) \otimes_{\mathbb{Z}} \mathbb{C}\right)$ has cardinality $n$ ! (resp. $\left.(n !)^{2}\right)$. We call $\pi$ algebraic if $z_{\pi} \in \rho+X^{*}(T)$ (resp. $\left.\widetilde{\rho}+X^{*}\left(\widetilde{T} \times_{\mathbb{R}} \mathbb{C}\right)\right)$, where

$$
\rho=((n-1) / 2,(n-3) / 2, \ldots,(1-n) / 2)
$$

and $\widetilde{\rho}=(\rho, \rho)$. Then $\pi$ has the same infinitesimal character as an irreducible algebraic representation of $G L_{n}$ (resp. the restriction of scalars of $G L_{n}$ from $\mathbb{C}$ to $\mathbb{R}$ ) if and only if $\pi$ is regular and algebraic.

If $\pi$ is an irreducible admissible $\left(\left(\right.\right.$ Lie $\left.\left.G L_{n}(\mathbb{R})\right) \otimes_{\mathbb{R}} \mathbb{C}, O(n)\right)$-module and if $\pi_{\mathbb{C}}$ denotes its base change to $\mathbb{C}$ then

$$
z_{\pi_{\mathbb{C}}}=\left(z_{\pi}, z_{\pi}\right)
$$


If $\pi_{i}$ is an irreducible admissible $\left(\left(\operatorname{Lie} G L_{n_{i}}(\mathbb{R})\right) \otimes_{\mathbb{R}} \mathbb{C}, O\left(n_{i}\right)\right)$-module (resp. $\left(\left(\right.\right.$ Lie $\left.\left.G L_{n_{i}}(\mathbb{C})\right) \otimes_{\mathbb{R}} \mathbb{C}, U\left(n_{i}\right)\right)$-module) for $i=1,2$ then $z_{\pi_{1} \boxplus \pi_{2}}$ is the image of

$$
\left(z_{\pi_{1}}, z_{\pi_{2}}\right) \in\left(\mathbb{Z}^{n_{1}} / S_{n_{1}}\right) \times\left(\mathbb{Z}^{n_{2}} / S_{n_{2}}\right)
$$

(resp. $\left.\left(\left(\mathbb{Z}^{n_{1}} \oplus \mathbb{Z}^{n_{1}}\right) /\left(S_{n_{1}} \times S_{n_{1}}\right)\right) \times\left(\left(\mathbb{Z}^{n_{2}} \oplus \mathbb{Z}^{n_{2}}\right) /\left(S_{n_{2}} \times S_{n_{2}}\right)\right)\right)$ in $\mathbb{Z}^{n_{1}+n_{2}} / S_{n_{1}+n_{2}}$ (resp. $\left(\left(\mathbb{Z}^{n_{1}+n_{2}} \oplus \mathbb{Z}^{n_{1}+n_{2}}\right) /\left(S_{n_{1}+n_{2}} \times S_{n_{1}+n_{2}}\right)\right)$ ).

The following observation will be very important for us.

Lemma 7.1. Suppose that $\pi$ is an irreducible, unitary, admissible $\left(\left(\operatorname{Lie} G L_{n}(\mathbb{C})\right)\right.$ $\left.\otimes_{\mathbb{R}} \mathbb{C}, U(n)\right)$-module and that $z_{\pi} \in(1 / 2) X^{*}\left(\widetilde{T} \times_{\mathbb{R}} \mathbb{C}\right)$. Then

$$
\pi^{c} \cong \pi^{\vee} \text {. }
$$

Proof. It follows from the classification of irreducible, unitary, admissible $\left(\left(\right.\right.$ Lie $\left.\left.G L_{n}(\mathbb{C})\right) \otimes_{\mathbb{R}} \mathbb{C}, U(n)\right)$-modules in $\left.\mathrm{Tad}\right]$ that $\pi$ is the full normalized induction of a unitary character of some parabolic subgroup of $G L_{n}(\mathbb{C})$. The lemma is then immediate.

We will also need a slight strengthening of the notion of 'big' introduced in CHT.

Definition 7.2. Let $k / \mathbb{F}_{l}$ be algebraic and let $m$ be a positive integer. We will call a subgroup $H \subset G L_{n}(k) m$-big if the following conditions are satisfied:

- $H$ has no $l$-power order quotient.

- $H^{0}\left(H, \mathfrak{g l}_{n}^{0}(k)\right)=(0)$.

- $H^{1}\left(H, \mathfrak{g l}_{n}^{0}(k)\right)=(0)$.

- For all irreducible $k[H]$-submodules $W$ of $\mathfrak{g l}_{n}(k)$ we can find $h \in H$ and $\alpha \in k$ with the following properties:

- the element $\alpha$ is a simple root of the characteristic polynomial of $h$ and if $\beta$ is another root then $\alpha^{m} \neq \beta^{m}$;

$-\pi_{h, \alpha} \circ W \circ i_{h, \alpha} \neq(0)$.

We will use big as a synonym for 1-big. (This agrees with the terminology of [CHT.)

The criteria given in Section 2.5 of [CHT] for a subgroup $H \subset G L_{n}(k)$ to be big, easily generalise to criteria for $H$ to be $m$-big. We state just two examples. The proofs are so similar to those of Lemma 2.5.2 and Corollary 2.5.3 of [CHT] we do not give them here.

Lemma 7.3. Keep the notation of the definition and assume that $l>$ $2(n-1) m+1$. Suppose also that 
- H has no l-power order quotient;

- $H$ contains $\operatorname{Symm}^{n-1} S L_{2}\left(\mathbb{F}_{l}\right)$;

- $H^{1}\left(H, \mathfrak{g l}_{n}^{0}(k)\right)=(0)$.

Then $H$ is m-big.

Lemma 7.4. Let $m, n$ and $d$ be positive integers. Then there is a constant $C$ (depending on $m, n$ and $d$ ) with the following properties. If $l>C$ is a prime and if $k / \mathbb{F}_{l}$ is an extension of degree $\leq d$ and if $H \subset G L_{n}(k)$ is a subgroup satisfying

- H has no l-power order quotient;

- $H$ contains $\mathrm{Symm}^{n-1} S L_{2}\left(\mathbb{F}_{l}\right)$,

then $H$ is $m$-big.

We now turn to the main theorems of this section, first the ordinary case.

Theorem 7.5. Suppose that $F$ is a totally real field and that $n$ is a positive integer. Suppose that $l>2 n$ is a rational prime and that $L / \mathbb{Q}_{l}$ is a finite extension with ring of integers $\mathcal{O}$, maximal ideal $\lambda$ and residue field $\mathbb{F}$. Suppose also that

$$
r: \operatorname{Gal}(\bar{F} / F) \rightarrow G L_{n}(\mathcal{O})
$$

is a continuous representation which is unramified at all but finitely many primes. Suppose also that there is a perfect pairing

$$
\langle,\rangle: L^{n} \times L^{n} \rightarrow L
$$

and a character

$$
\mu: \operatorname{Gal}(\bar{F} / F) \rightarrow \mathcal{O}^{\times}
$$

such that

$$
\langle g x, g y\rangle=\mu(g)\langle x, y\rangle
$$

for all $x, y \in L^{n}$ and all $g \in \operatorname{Gal}(\bar{F} / F)$. Let $\bar{r}$ denote the semisimplification of the reduction of $r$ modulo $\lambda$. Suppose moreover that $r$ enjoys the following properties.

1. There is an $\varepsilon \in\{ \pm 1\}$ such that

- for every place $v \mid \infty$ of $F$ we have $\mu\left(c_{v}\right)=\varepsilon$;

- for every $x, y \in L^{n}$ we have $\langle y, x\rangle=\varepsilon\langle x, y\rangle$.

2. $\left[\bar{F}^{\text {ker ad } \bar{r}}\left(\zeta_{l}\right): \bar{F}^{\text {ker ad } \bar{r}}\right]>2$.

3. The image $\bar{r}\left(\operatorname{Gal}\left(\bar{F} / F\left(\zeta_{l}\right)\right)\right)$ is 2-big $\left(\right.$ in $\left.G L_{n}\left(\overline{\mathbb{F}}_{l}\right)\right)$.

4. $r$ is ordinary of weight a for some $a \in\left(\mathbb{Z}^{n}\right)^{\operatorname{Hom}\left(F, \overline{\mathbb{Q}}_{l}\right),+}$. 
Then there is a Galois totally real extension $F^{\prime} / F$ such that $\left.r\right|_{\mathrm{Gal}\left(\bar{F} / F^{\prime}\right)}$ is automorphic of weight $a_{F^{\prime}}$.

Proof. The strategy is to tensor $r$ with a representation $r^{\prime}$ induced from a character $\theta$ of $M F$, where $M$ is a suitable imaginary quadratic field. The character $\theta$ is chosen so that there is a perfect pairing on $r^{\prime}$ with the opposite parity to the given pairing on $r$, and so that the tensor product $r^{\prime \prime}=r \otimes r^{\prime}$ has multiplier a power of the cyclotomic character. We then apply Theorem 6.3 to $r^{\prime \prime}$. Much of the proof will be devoted to checking that $\bar{r}^{\prime \prime}(\operatorname{Gal}(\bar{F} / F))$ is big. Finally we use the theory of base change to deduce the automorphy of $r$ from the automorphy of $r^{\prime \prime}$.

Step 1: Choice of $b, M, q, \phi, \theta, r^{\prime}$. As $\bar{r}$ is absolutely irreducible, the dual lattice of $\mathcal{O}^{n}$ in $L^{n}$ under $\langle$,$\rangle is \lambda^{m} \mathcal{O}^{n}$ for some integer $m$. Replacing $\langle$,$\rangle by \alpha\langle$,$\rangle ,$ where $\lambda^{m}=(\alpha)$, we may suppose that it induces a perfect duality on $\mathcal{O}^{n}$.

Choose an integer $b$ strictly greater than $a_{\tau, 1}-a_{\tau, n}$ for all $\tau: F \hookrightarrow \overline{\mathbb{Q}}_{l}$. Define $a^{\prime \prime} \in\left(\mathbb{Z}^{2 n}\right)^{\operatorname{Hom}\left(F, \overline{\mathbb{Q}}_{l}\right),+}$ by

$$
a_{\tau}^{\prime \prime}=\left(a_{\tau, 1}+b, \ldots, a_{\tau, n}+b, a_{\tau, 1}, \ldots, a_{\tau, n}\right) .
$$

Also choose an imaginary quadratic field $M$ which is linearly disjoint from $\bar{F}^{\text {ker } \bar{r}}\left(\zeta_{l}\right)$ over $\mathbb{Q}$ and in which $l$ splits. Let $\delta_{M}$ denote the quadratic character of $\operatorname{Gal}(\bar{F} / \mathbb{Q})$ corresponding to $M / \mathbb{Q}$. Choose a rational prime $q$ which splits in $M$, is unramified in $\bar{F}^{\text {ker } r}\left(\zeta_{4 l}\right)$ and does not divide $\# \bar{r}(\operatorname{Gal}(\bar{F} / F))$. Also choose a prime $\mathfrak{q}$ of $M$ above $q$. Choose a CM field $M_{0}$ and a prime $\lambda_{0}$ of $M_{0}$ above $l$ and a continuous embedding $L \hookrightarrow M_{0, \lambda_{0}}$ and a continuous character

$$
\psi: \operatorname{Gal}(\bar{F} / F) \rightarrow \mathcal{O}_{M_{0}}^{\times} \quad \text { with } \quad \psi \equiv \mu^{-1} \epsilon_{l}^{b+1-2 n} \delta_{M}^{(1+\varepsilon) / 2} \bmod \lambda_{0} .
$$

Choose a finite $\mathrm{CM}$ extension $M^{\prime} / M M_{0}$ and a character $\phi: \mathbb{A}_{M F}^{\times} \rightarrow\left(M^{\prime}\right)^{\times}$with open kernel such that

- if $\alpha \in(M F)^{\times}$then $\phi(\alpha)=\left(\mathbf{N}_{M F / M} \alpha\right)^{b}$;

- $\left.\phi\right|_{\mathbb{A}_{F}^{\times}}=\left(\prod_{v \nmid \infty}|\cdot|_{v}^{-b}\right)\left(\prod_{v \mid \infty} \operatorname{sgn}_{v}^{b}\right)\left(\psi \circ \operatorname{Art}_{F}\right)$;

- $q \mid \# \phi\left(\mathcal{O}_{F M, \mathfrak{q}}^{\times}\right)$.

(See Lemma 2.2 of [HSBT].) Choose a prime $\lambda^{\prime}$ of $M^{\prime}$ above $\lambda_{0}$. Let

$$
\theta: \operatorname{Gal}(\bar{F} / F M) \rightarrow \mathcal{O}_{M^{\prime}, \lambda^{\prime}}^{\times}
$$

be the character defined by

$$
\theta\left(\operatorname{Art}_{F M} \alpha\right)=\phi(\alpha)\left(\mathbf{N}_{F M / M} \alpha\right)_{\left.\lambda^{\prime}\right|_{M}}^{-b}
$$


The induced representation

$$
r^{\prime}=\operatorname{Ind}_{\operatorname{Gal}(\bar{F} / F)}^{\operatorname{Gal}(\bar{F})} \theta
$$

has a basis $e_{1}, e_{2}$ such that $r^{\prime}(c) e_{1}=e_{2}$ and $r^{\prime}(c) e_{2}=e_{1}$, and for $\sigma \in \operatorname{Gal}(\bar{F} / M)$,

$$
r^{\prime}(\sigma) e_{1}=\theta(\sigma) e_{1} \quad \text { and } \quad r^{\prime}(\sigma) e_{2}=\theta(c \sigma c) e_{2} .
$$

Define a perfect pairing $\langle,\rangle^{\prime}$ on $r^{\prime}$ by setting

$$
\begin{gathered}
\left\langle e_{i}, e_{i}\right\rangle^{\prime}=0 \quad \text { for } i=1,2, \\
\left\langle e_{1}, e_{2}\right\rangle^{\prime}=1, \quad\left\langle e_{2}, e_{1}\right\rangle^{\prime}=-\varepsilon .
\end{gathered}
$$

Then

$$
\left\langle r^{\prime}(\sigma) x, r^{\prime}(\sigma) y\right\rangle^{\prime}=\epsilon_{l}(\sigma)^{-b} \psi(\sigma) \delta_{M}(\sigma)^{\left(1+\mu\left(c_{v}\right)\right) / 2}\langle x, y\rangle
$$

for all $x, y \in\left(M_{\lambda^{\prime}}^{\prime}\right)^{2}$ and all $\sigma \in \operatorname{Gal}(\bar{F} / \mathbb{Q})$. Let $f_{1}, f_{2}$ denote the basis of $\operatorname{Hom}\left(r^{\prime}, \mathcal{O}_{M^{\prime}, \lambda^{\prime}}\right)$ dual to $e_{1}, e_{2}$.

Now define

$$
r^{\prime \prime}=r \otimes r^{\prime}: \operatorname{Gal}(\bar{F} / F) \rightarrow G S p_{2 n}\left(\mathcal{O}_{M^{\prime}, \lambda^{\prime}}\right) .
$$

It has multiplier $\mu \epsilon_{l}^{-b} \psi \delta_{M}^{\left(1+\mu\left(c_{v}\right)\right) / 2}$ which is congruent to $\epsilon_{l}^{1-2 n}$ modulo $\lambda^{\prime}$. Let $\bar{r}^{\prime}$ (resp. $\bar{r}^{\prime \prime}$ ) denote the reduction of $r^{\prime}$ (resp. $r^{\prime \prime}$ ) modulo $\lambda^{\prime}$.

Step 2: Automorphy of $r^{\prime \prime}=r \otimes r^{\prime}$. As the primes that ramify in $\bar{F}^{\mathrm{ker} \bar{r}}\left(\zeta_{l}\right) / \mathbb{Q}$ and in $M / \mathbb{Q}$ are disjoint sets we may find $\sigma \in \operatorname{Gal}\left(\bar{F} / \bar{F}^{\text {ker ad } \bar{r}} M\right)$ so that $\epsilon_{l}(\sigma) \bmod l$ has order greater than 2 . Let

$$
\sigma^{\prime}=\sigma\left(c_{v} \sigma c_{v}\right)
$$

Then $\operatorname{ad} \bar{r}^{\prime \prime}\left(\sigma^{\prime}\right)=1$ while $\epsilon_{l}\left(\sigma^{\prime}\right)=\epsilon_{l}(\sigma)^{2}$ is not equivalent to 1 modulo $l$. Thus $\zeta_{l} \notin \bar{F}^{\text {ker ad } \bar{r}^{\prime \prime}}$.

Let $H$ (resp. $H^{\prime}$, resp. $\left.H^{\prime \prime}\right)$ denote the image $(\operatorname{ad} \bar{r}) \operatorname{Gal}\left(\bar{F} / F\left(\zeta_{l}\right)\right)$ (resp. $\left(\operatorname{ad} \bar{r}^{\prime}\right) \operatorname{Gal}\left(\bar{F} / F\left(\zeta_{l}\right)\right)$, resp. $\left.\left(\operatorname{ad} \bar{r}^{\prime \prime}\right) \operatorname{Gal}\left(\bar{F} / F\left(\zeta_{l}\right)\right)\right)$. Note that the only scalar element of $H$ or $H^{\prime}$ (thought of as subgroups of Aut $(\operatorname{ad} \bar{r})$ and $\left.\operatorname{Aut}\left(\operatorname{ad} \bar{r}^{\prime}\right)\right)$ is the identity (look at the action of such an element on $1_{n}$ ) and so

$$
H \times H^{\prime} \hookrightarrow \operatorname{Aut}\left(\operatorname{ad} \bar{r}^{\prime \prime}\right) .
$$

Thus there is a finite group $\bar{H}$ and surjections $\pi: H \rightarrow \bar{H}$ and $\pi^{\prime}: H^{\prime} \rightarrow \bar{H}$ so that $H^{\prime \prime}$ is the set of elements $\left(h, h^{\prime}\right) \in H \times H^{\prime}$ such that $\pi(h)=\pi^{\prime}\left(h^{\prime}\right)$. Let $K$ (resp. $K^{\prime}$ ) denote the kernel of $\pi$ (resp. $\pi^{\prime}$ ). Note that the image of the inertia group at any prime above $q$ in $H^{\prime}$ is contained in $K^{\prime}$ (as $\bar{r}$ is unramified at $q$ ). Also 
note that the restriction of $H^{\prime} \rightarrow \operatorname{Gal}\left(M F\left(\zeta_{l}\right) / F\left(\zeta_{l}\right)\right)$ to $K^{\prime}$ is surjective (as $M$ is linearly disjoint from $\bar{F}^{\operatorname{ker} \bar{r}}\left(\zeta_{l}\right)$ over $\left.\mathbb{Q}\right)$.

Write

$$
\operatorname{ad} \bar{r} \otimes_{\mathbb{F}} \overline{\mathbb{F}}_{l}=V_{0}^{\oplus m_{0}} \oplus V_{1}^{\oplus m_{1}} \oplus \cdots \oplus V_{s}^{\oplus m_{s}},
$$

with $1=V_{0}, V_{1}, \ldots, V_{s}$ pairwise non-isomorphic, irreducible $\overline{\mathbb{F}}_{l}\left[\operatorname{Gal}\left(\bar{F} / F\left(\zeta_{l}\right)\right)\right]$ modules, and with $1=m_{0}, m_{1}, \ldots, m_{s}$ positive integers. Then

$$
\operatorname{ad} \bar{r}^{\prime \prime} \otimes_{\mathcal{O}_{M^{\prime}} / \lambda^{\prime}} \overline{\mathbb{F}}_{l}=\bigoplus_{j=0}^{s}\left(V_{j}^{m_{j}} \oplus\left(V_{j} \otimes \delta_{M}\right)^{m_{j}} \oplus\left(\operatorname{Ind}_{\operatorname{Gal}\left(\bar{F} / F M\left(\zeta_{l}\right)\right)}^{\operatorname{Gal}\left(\bar{F} / F\left(\zeta_{l}\right)\right)}\left(V_{j} \otimes \bar{\theta} / \bar{\theta}^{c}\right)\right)^{m_{j}}\right) .
$$

Here we identify $V_{j}$ with $V_{j} \otimes\left(e_{1} \otimes f_{1}+e_{2} \otimes f_{2}\right) ; V_{j} \otimes \delta_{M}$ with $V_{j} \otimes\left(e_{1} \otimes f_{1}-e_{2} \otimes f_{2}\right)$; and $\operatorname{Ind}_{\operatorname{Gal}\left(\bar{F} / F M\left(\zeta_{l}\right)\right)}^{\left.\operatorname{Gal}\left(\overline{C_{l}}\right)\right)}\left(W_{j} \otimes \bar{\theta} / \bar{\theta}^{c}\right)$ with the span of $V_{j} \otimes\left(e_{1} \otimes f_{2}\right)$ and $V_{j} \otimes\left(e_{2} \otimes f_{1}\right)$. These constituents are all irreducible as $\left.V_{j}\right|_{\mathrm{Gal}\left(\bar{F} / F M\left(\zeta_{l}\right)\right)}$ is irreducible and $V_{j} \neq$ $V_{j} \otimes\left(\bar{\theta} / \bar{\theta}^{c}\right)^{2}$ as $\overline{\mathbb{F}}_{l}\left[\operatorname{Gal}\left(\bar{F} / F M\left(\zeta_{l}\right)\right)\right]$-modules (as one is ramified above $q$ and the other is not). In particular

$$
H^{0}\left(\left(\operatorname{ad} \bar{r}^{\prime \prime}\right)\left(\operatorname{Gal}\left(\bar{F} / F\left(\zeta_{l}\right)\right)\right), \operatorname{ad}^{0} \bar{r}^{\prime \prime}\right)=(0)
$$

(as no $V_{j}$ is isomorphic to $\delta_{M}$ as $M$ is linearly disjoint from $\bar{F}^{\mathrm{ker} \bar{r}}$ ). Moreover

$$
(0)=H^{1}\left(H, \operatorname{ad}^{0} \bar{r}\right)=H^{1}\left(H^{\prime \prime} / K^{\prime},\left(\operatorname{ad}^{0} \bar{r}^{\prime \prime}\right)^{K^{\prime}}\right) \stackrel{\sim}{\rightarrow} H^{1}\left(H^{\prime \prime}, \operatorname{ad}^{0} \bar{r}^{\prime \prime}\right)
$$

$\left(\right.$ as $\left.l \nmid \# K^{\prime}\right)$.

Fix a copy $V_{j} \subset \operatorname{ad} \bar{r} \otimes_{\mathbb{F}} \overline{\mathbb{F}}_{l}$. We can find a $\sigma \in \operatorname{Gal}\left(\bar{F} / M F\left(\zeta_{l}\right)\right)$ and a simple root $\alpha$ of the characteristic polynomial of $\bar{r}(\sigma)$ such that $\pi_{\bar{r}(\sigma), \alpha} V_{j} i_{\bar{r}(\sigma), \alpha} \neq(0)$. Altering $\sigma$ by elements of inertia subgroups at primes above $q$ we may further suppose that the ratio $\left(\bar{\theta} / \bar{\theta}^{c}\right)(\sigma)$ does not equal $\alpha^{\prime} / \alpha$ for any root $\alpha^{\prime}$ (including $\alpha$ ) of the characteristic polynomial of $\bar{r}(\sigma)$ (as $q \mid \#\left(\bar{\theta} / \bar{\theta}^{c}\right)\left(I_{M_{\mathrm{q}}}\right)$ and $\left.q>n\right)$. Then $\alpha \bar{\theta}(\sigma)$ is a simple root of the characteristic polynomial of $\bar{r}^{\prime \prime}(\sigma)$ and

$$
\begin{aligned}
& \pi_{\bar{r}^{\prime \prime}(\sigma), \alpha \bar{\theta}(\sigma)} V_{j} \otimes\left(e_{1} \otimes f_{1} \pm e_{2} \otimes f_{2}\right) i_{\bar{r}^{\prime \prime}(\sigma), \alpha \bar{\theta}(\sigma)} \\
& \quad=\pi_{\bar{r}^{\prime \prime}(\sigma), \alpha \bar{\theta}(\sigma)} V_{j} \otimes\left(e_{1} \otimes f_{1}\right) i_{\bar{r}^{\prime \prime}(\sigma), \alpha \bar{\theta}(\sigma)}=\pi_{\bar{r}(\sigma), \alpha} V_{j} i_{\bar{r}(\sigma), \alpha} \neq(0)
\end{aligned}
$$

for both signs \pm .

Continue to fix a copy $V_{j} \subset$ ad $\bar{r} \otimes_{\mathbb{F}} \overline{\mathbb{F}}_{l}$. Choose $\sigma \in \operatorname{Gal}\left(\bar{F} / F\left(\zeta_{l}\right)\right)$ and a simple root $\alpha$ of the characteristic polynomial of $\bar{r}(\sigma)$ such that

- $\sigma$ maps to $c \in \operatorname{Gal}\left(M F\left(\zeta_{l}\right) / F\left(\zeta_{l}\right)\right)$;

- $\pi_{\bar{r}(\sigma), \alpha} V_{j} i_{\bar{r}(\sigma), \alpha} \neq(0)$;

- $-\alpha$ is not a root of the characteristic polynomial of $\bar{r}(\alpha)$. 
(We are using the assumption that $\bar{r}\left(\mathrm{Gal}\left(\bar{F} / F\left(\zeta_{l}\right)\right)\right)$ is 2-big.) Write

$$
\bar{r}^{\prime}(\sigma) e_{i}=\beta_{i} e_{i+1}
$$

for $i=0,1$ (where we take $e_{2}=e_{0}$ ). The roots of the characteristic polynomial of $\bar{r}^{\prime}(\sigma)$ are exactly $\pm \beta$ where $\beta^{2}=\beta_{0} \beta_{1}$ and corresponding eigenvectors are $e_{0} \pm \beta_{0} / \beta e_{1}$. Then $\alpha \beta$ is a simple root of the characteristic polynomial of $\bar{r}^{\prime \prime}(\sigma)$ and

$$
\pi_{\bar{r}^{\prime \prime}(\sigma), \alpha \beta}\left(v \otimes e_{0} \otimes f_{1}\right) i_{\bar{r}^{\prime \prime}(\sigma), \alpha \beta}=\left(\beta / 2 \beta_{1}\right) \pi_{\bar{r}(\sigma), \alpha} v i_{\bar{r}(\sigma), \alpha} .
$$

For some choice of $v$ this will be non-zero. We conclude that $\bar{r}^{\prime \prime}\left(\operatorname{Gal}\left(\bar{F} / F\left(\zeta_{l}\right)\right)\right)$ is big.

It follows from Theorem 6.3 that there is a Galois totally real extension $F^{\prime} / F$, an isomorphism $\imath: \overline{M_{\lambda^{\prime}}^{\prime}} \stackrel{\sim}{\rightarrow} \mathbb{C}$ and a RAESDC automorphic representation $\Pi$ of $G L_{2 n}\left(\mathbb{A}_{F^{\prime}}\right)$ of weight $\imath_{*} a^{\prime \prime}$ and such that $r_{l, \imath}(\Pi) \cong r^{\prime \prime}$.

Step 3: Automorphy of $r$. We see that $\Pi \otimes\left(\delta_{M} \circ \operatorname{Art}_{F^{\prime}}\right) \cong \Pi$, and hence by Theorem 4.2 of $\mathrm{AC}$ ] there is a cuspidal automorphic representation $\pi$ of $G L_{n}\left(\mathbb{A}_{F^{\prime} M}\right)$ such that for all places $v$ of $F^{\prime} M$ the representation $\pi_{v} \boxplus\left(\pi^{c}\right)_{v}$ is the base change of $\Pi_{\left.v\right|_{F^{\prime}}}|\cdot|_{\left.v\right|_{F^{\prime}}}^{-n / 2}$ from $F_{\left.v\right|_{F^{\prime}}}^{\prime}$ to $\left(F^{\prime} M\right)_{v}$. Note that for $v \mid \infty$ the representation $\pi_{v}$ is regular algebraic.

There is also an algebraic Hecke character $\chi$ of $\mathbb{A}_{F^{\prime}}^{\times} /\left(F^{\prime}\right)^{\times}$such that $\Pi^{\vee}=$ $\Pi \otimes\left(\left(|\cdot|^{-n} \chi\right) \circ\right.$ det $)$. From the classification of algebraic grossencharacters we know that there is an integer $w$ such that $\chi|\cdot|^{-w}$ has finite image. It follows that $\pi^{\vee} \cong \pi \otimes\left(\chi \circ \mathbf{N}_{F^{\prime} M / F^{\prime}} \circ\right.$ det $)$ or $\pi^{c}\left(\chi \circ \mathbf{N}_{F^{\prime} M / F^{\prime}} \circ\right.$ det $)$. We wish to exclude the first possibility. If it held then $\pi \otimes\left(|\cdot|^{w / 2} \circ\right.$ det $)$ would have unitary central character and so would itself be unitary. Also, for $v \mid \infty$, the infinitesimal character of $\pi_{v} \otimes\left(|\cdot|_{v}^{w / 2} \circ\right.$ det$)$ has Harish-Chandra parameter lying in $(1 / 2) X^{*}\left(\widetilde{T} \times_{\mathbb{R}} \mathbb{C}\right)$. Hence using Lemma 7.1 we would see that

$$
\begin{aligned}
\pi_{v} \boxplus \pi_{v}^{c} & \cong \pi_{v} \boxplus\left(\left(\pi_{v} \otimes\left(|\cdot|^{w / 2} \circ \operatorname{det}\right)\right)^{c} \otimes\left(|\cdot|^{-w / 2} \circ \operatorname{det}\right)\right) \\
& \cong \pi_{v} \boxplus\left(\pi_{v}^{\vee} \otimes\left(|\cdot|^{-w} \circ \operatorname{det}\right)\right) \\
& \cong \pi_{v} \boxplus\left(\pi_{v} \otimes\left(\left(\chi|\cdot|^{-w}\right) \circ \mathbf{N}_{M F^{\prime} / F^{\prime}} \circ \operatorname{det}\right)\right),
\end{aligned}
$$

which would contradict the regularity of $\Pi_{\left.v\right|_{F^{\prime}}}$. We conclude that

$$
\pi^{\vee} \cong \pi^{c}\left(\chi \circ \mathbf{N}_{F^{\prime} M / F^{\prime}} \circ \operatorname{det}\right)
$$

Thus $\pi$ is a RAECSDC automorphic representation of $G L_{n}\left(\mathbb{A}_{F^{\prime} M}\right)$ and it has an associated Galois representation $r_{l, \imath}(\pi)$.

We see that

$$
\left.\left.r_{l, \imath}(\pi) \oplus r_{l, \imath}(\pi)^{c} \cong r_{l, \imath}(\Pi)\right|_{\operatorname{Gal}\left(\bar{F} / F^{\prime} M\right)} \cong\left(\theta \oplus \theta^{c}\right) \otimes r\right|_{\operatorname{Gal}\left(\bar{F} / F^{\prime} M\right)} .
$$


Writing $\theta=r_{l, \imath}(\widetilde{\theta})$ we see that

$$
\left.r\right|_{\operatorname{Gal}\left(\bar{F} / F^{\prime} M\right)} \cong r_{l, \imath}\left(\pi \otimes(\widetilde{\theta} \circ \text { det })^{-1}\right) \quad \text { or } \quad r_{l, l}\left(\pi^{c} \otimes(\widetilde{\theta} \circ \text { det })^{-1}\right)
$$

In either case the theorem follows from Lemma 1.5

We now turn to the 'niveau two' case, where we are only able to prove a much weaker theorem.

Theorem 7.6. Suppose that $F$ is a totally real field; that $n$ and $b$ are positive integers; that $T$ is a finite set of rational primes and that $\mu: \operatorname{Gal}(\bar{F} / F) \rightarrow \overline{\mathbb{Q}}^{\times}$is a continuous character. Then we can find a Galois $C M$ extension $M^{\prime} / \mathbb{Q}$ containing the image of $\mu$ and a finite set $S$ of rational primes, containing all primes that ramify in $M^{\prime}$, with the following properties.

Suppose that $l \notin S$ is a rational prime with $\left[\mathrm{Frob}_{l}\right]=[c] \subset \operatorname{Gal}\left(M^{\prime} / \mathbb{Q}\right)$ and that $\lambda^{\prime} \mid l$ is a prime of $M^{\prime}$ (so that $\left.M_{\lambda^{\prime}} \cong \mathbb{Q}_{l^{2}}\right)$. Suppose also that $r: \operatorname{Gal}(\bar{F} / F) \rightarrow$ $G L_{n}\left(\mathcal{O}_{M^{\prime}, \lambda^{\prime}}\right)$ is a continuous representation which is unramified outside $T \cup\{l\}$. Suppose also that there is a perfect pairing

$$
\langle,\rangle:\left(M_{\lambda^{\prime}}^{\prime}\right)^{n} \times\left(M_{\lambda^{\prime}}^{\prime}\right)^{n} \rightarrow M_{\lambda^{\prime}}^{\prime}
$$

such that

$$
\langle g x, g y\rangle=\epsilon_{l}(g)^{(1-n) b} \mu(g)\langle x, y\rangle
$$

for all $x, y \in L^{n}$ and all $g \in \operatorname{Gal}(\bar{F} / F)$. Let $\bar{r}$ denote the semisimplification of the reduction of $r$ modulo the maximal ideal of $\mathcal{O}$. Suppose moreover that $r$ enjoys the following properties:

1. There is an $\varepsilon \in\{ \pm 1\}$ such that

- for every place $v \mid \infty$ of $F$ we have $\mu\left(c_{v}\right)=\varepsilon(-1)^{(1-n) b}$;

- for all $x, y \in L^{n}$ we have $\langle y, x\rangle=\varepsilon\langle x, y\rangle$.

2. $c \circ r \cong r \otimes \mu^{-1}$.

3. $\left[\bar{F}^{\text {ker ad } \bar{r}}\left(\zeta_{l}\right): \bar{F}^{\text {ker ad } \bar{r}}\right]>2 b$.

4. The image $\bar{r}\left(\operatorname{Gal}\left(\bar{F} / F\left(\zeta_{l}\right)\right)\right)$ is 2b-big $\left(\right.$ in $\left.G L_{n}\left(\overline{\mathbb{F}}_{l}\right)\right)$.

5. - $l$ is unramified in $F$;

- if $v \mid l$ is a prime of $F$ then $r$ is crystalline at $v$;

- if $v \mid l$ is a prime of $F$ then

$$
\left.\bar{r}\right|_{I_{F_{v}}} \sim \omega_{2}^{(1-n) b} \oplus \omega_{2}^{(-l+2-n) b} \oplus \omega_{2}^{(-2 l+3-n) b} \oplus \cdots \oplus \omega_{2}^{(1-n) l b} ;
$$

- if $v \mid l$ is a prime of $F$ and if $\tau: F_{v} \hookrightarrow \bar{M}_{\lambda^{\prime}}^{\prime}$ then

$$
\operatorname{dim}_{\bar{M}_{\lambda^{\prime}}^{\prime}} \operatorname{gr}^{i}\left(\left(r \otimes_{M_{\lambda^{\prime}}^{\prime}} \bar{M}_{\lambda^{\prime}}^{\prime}\right) \otimes_{\tau, F_{v}} B_{\mathrm{DR}}\right)^{\operatorname{Gal}\left(\bar{F}_{v} / F_{v}\right)}=0
$$


unless $i=(n-j) b$ for some $j=1, \ldots, n$ in which case

$$
\operatorname{dim}_{\bar{M}_{\lambda^{\prime}}^{\prime}} \operatorname{gr}^{i}\left(\left(r \otimes_{M_{\lambda^{\prime}}^{\prime}} \bar{M}_{\lambda^{\prime}}^{\prime}\right) \otimes_{\tau, F_{v}} B_{\mathrm{DR}}\right)^{\operatorname{Gal}\left(\bar{F}_{v} / F_{v}\right)}=1 .
$$

Then there is a Galois totally real extension $F^{\prime} / F$ such that $\left.r\right|_{\mathrm{Gal}\left(\bar{F} / F^{\prime}\right)}$ is automorphic.

Proof. The strategy is much the same as for the proof of Theorem 7.5. We will again tensor $r$ with a representation $r^{\prime}$ induced from a character $\theta$ of $M F$, where $M / \mathbb{Q}$ is an imaginary cyclic CM extension of degree $2 b$. The character $\theta$ is chosen so that there is a perfect pairing on $r^{\prime}$ with the opposite parity to the given pairing on $r$, and so that the tensor product $r^{\prime \prime}=r \otimes r^{\prime}$ has multiplier a power of the cyclotomic character. In this case the character $\theta$ must also be chosen so that $r^{\prime \prime}$ has consecutive Hodge-Tate numbers. We then apply Theorem 6.4 to $r^{\prime \prime}$. Much of the proof will be devoted to checking that $\bar{r}^{\prime \prime}(\operatorname{Gal}(\bar{F} / F))$ is big. Finally we use the theory of base change to deduce the automorphy of $r$ from the automorphy of $r^{\prime \prime}$.

One complication in this case is that we must ensure that $r^{\prime \prime}$ is defined over $\mathbb{Q}_{l}$, and not some extension. This is the reason for choosing a Galois CM extension $M^{\prime} / \mathbb{Q}$ at the start and requiring that $\left[\operatorname{Frob}_{l}\right]=[c] \subset \operatorname{Gal}\left(M^{\prime} / \mathbb{Q}\right)$. We choose $M^{\prime}$ so that there is a continuous character $\phi: \mathbb{A}_{M}^{\times} \rightarrow\left(M^{\prime}\right)^{\times}$. Then for any prime $l$ with $\left[\operatorname{Frob}_{l}\right]=[c] \subset \operatorname{Gal}\left(M^{\prime} / \mathbb{Q}\right)$ we obtain (from $\left.\phi\right)$ a character $\theta: \operatorname{Gal}(\bar{F} / M) \rightarrow \mathbb{Q}_{l^{2}}^{\times}$. Thus $r^{\prime \prime}$ is at least defined over $\mathbb{Q}_{l^{2}}$. In the presence of the other assumption that we will require for $\phi$ and $\theta$, it turns out that $r^{\prime \prime}$ will be defined over $\mathbb{Q}_{l}$ if $c \circ r \cong r \otimes \mu^{-1}$. We remark that if $c \circ r \cong r \otimes \mu$ then there is a finite extension $\widetilde{F} / F$ such that $\operatorname{tr} r(\operatorname{Gal}(\bar{F} / \widetilde{F})) \subset \mathbb{Q}_{l}$. We also remark that this is not such a strange condition, for instance it is satisfied for the Galois representations attached to elliptic modular forms and their tensor powers. (More generally suppose that $\rho$ is a semisimple representation of the Galois group of a number field over the completion $N_{\lambda}$ of a CM field $N$ at a prime $\lambda$ with $c \lambda=\lambda$. Suppose also that $\rho$ is pure of some weight $w$ and essentially self-dual, say $\rho \cong \rho^{\vee} \otimes \psi$. Then $c \circ \rho \cong \rho^{\vee} \otimes \epsilon_{l}^{-w} \cong \rho \otimes\left(\epsilon_{l}^{-w} \psi^{-1}\right)$.)

Another complication is that to apply Theorem 6.4 we must arrange that $l \equiv-1 \bmod N_{2}$ for a suitable integer $N_{2}$, in particular $N_{2}$ should not be divisible by any primes at which $r^{\prime \prime}$ ramifies. Hence we specify at the outset of the theorem the set of primes $T$ where $r$ can ramify (excluding $l$ ), and choose a suitable integer $N$ (which will be the $N_{2}$ of Theorem 6.4 at the outset of this proof. We then choose $M^{\prime} \supset \mathbb{Q}\left(\zeta_{N}\right)$ so that the condition $\left[\mathrm{Frob}_{l}\right]=[c] \subset \operatorname{Gal}\left(M^{\prime} / \mathbb{Q}\right)$ implies that $l \equiv-1 \bmod N$.

Step 1: Choice of $N, M, q, \phi, M^{\prime}, S, \theta, r^{\prime}, r^{\prime \prime}$. As $\bar{r}$ is absolutely irreducible, the dual lattice of $\mathbb{Z}_{l^{2}}^{n}$ in $\mathbb{Q}_{l^{2}}^{n}$ under $\langle$,$\rangle is l^{m} \mathbb{Z}_{l^{2}}^{n}$ for some integer $m$. Replacing $\langle$, 
by $l^{m}\langle$,$\rangle we may suppose that it induces a perfect duality on \mathbb{Z}_{l^{2}}^{n}$. We may also suppose that $T$ contains 2 and all primes which ramify in $F$.

Choose an odd positive integer $N>2 n b+1$ only divisible by primes outside $T$. Choose a cyclic $\mathrm{CM}$ extension $M / \mathbb{Q}$ of degree $2 b$ in which all primes in $T$ are unramified; a generator $\tau$ of $\operatorname{Gal}(M / \mathbb{Q})$; and a generator $\kappa$ of $\operatorname{Gal}(M / \mathbb{Q})^{\vee}$. Note that $M$ is linearly disjoint from $F$ over $\mathbb{Q}$ as they ramify at disjoint sets of primes. Set $\delta_{M}=\kappa^{b}$. Also choose a rational prime $q$ such that

- $q \notin T$;

- $q$ splits completely in $M$;

- $q$ is unramified in $\bar{F}^{\operatorname{ker} \bar{r}}$

- $q-1>2 n$;

and a prime $\mathfrak{q}$ of $F M$ above $q$. Choose a finite CM extension $M^{\prime} / M\left(\zeta_{N}\right)$ and a character $\phi: \mathbb{A}_{M F}^{\times} \rightarrow\left(M^{\prime}\right)^{\times}$with open kernel such that

- $M^{\prime} / \mathbb{Q}$ is Galois;

- if $\alpha \in(F M)^{\times}$then

$$
\phi(\alpha)=\prod_{j=0}^{b-1}\left(\tau^{j}\left(\mathbf{N}_{F M / M} \alpha\right)\right)^{j}\left(\tau^{b+j}\left(\mathbf{N}_{F M / M} \alpha\right)\right)^{(n+1) b-1-j} ;
$$

- $\left.\phi\right|_{\mathbb{A}_{F M^{+}}^{\times}}=\left(\left.\prod_{v \nmid \infty}|\cdot|\right|_{v} ^{1-(n+1) b}\right)\left(\prod_{v \mid \infty} \operatorname{sgn}_{v}^{1-(n+1) b}\right)\left(\left(\mu \delta_{M}^{(1+\varepsilon) / 2}\right)^{-1} \circ \operatorname{Art}_{F M^{+}}\right)$, where we think of $\mu$ as valued in $M^{\prime}$ via some embedding $M^{\prime} \hookrightarrow \overline{\mathbb{Q}}_{l}$;

- $q \mid \# \phi\left(\mathcal{O}_{F M, \mathfrak{q}}^{\times}\right)$, but $\phi$ is unramified at primes above $q$ other than $\mathfrak{q}$ and $\mathfrak{q}^{c}$.

(See Lemma 2.2 of [HSBT].) Let $S$ denote the union of $T$, the set of primes $\leq 2 b n$, the set of primes which ramify in $\mathbb{Q}\left(\zeta_{N}\right)$ and the set of primes above which $\phi$ ramifies.

Note that

$$
(c \circ \phi) \phi \prod_{v \nmid \infty}|\cdot|_{v}^{(n+1) b-1}: \mathbb{A}_{F M}^{\times} /(F M)^{\times} \rightarrow\left(\left(M^{\prime}\right)_{\gg 0}^{+}\right)^{\times},
$$

where the subscript $\gg 0$ indicates the subset of totally positive elements. As $\left(\left(M^{\prime}\right)_{\gg 0}^{+}\right)^{\times}$has no finite subgroups except for $\{1\}$ we deduce that

$$
(c \circ \phi)=\left(\mu \circ \operatorname{Art}_{F M}\right) \phi^{c} .
$$

(In case it has caused confusion we point out that $\phi^{c}$ is short for $\phi \circ c$.)

Now suppose $\lambda^{\prime} \mid l$ are primes as in the theorem. Let

$$
\theta: \operatorname{Gal}(\bar{F} / F M) \rightarrow \mathcal{O}_{M^{\prime}, \lambda^{\prime}}^{\times}
$$


be the character defined by

$$
\theta\left(\operatorname{Art}_{F M} \alpha\right)=\phi(\alpha) \prod_{j=0}^{b-1}\left(\tau^{j}\left(\mathbf{N}_{F M / M} \alpha_{l}\right)\right)^{-j}\left(\tau^{n / 2+j}\left(\mathbf{N}_{F M / M} \alpha_{l}\right)\right)^{1+j-(n+1) b},
$$

so that

$$
c \circ \theta=\mu \theta^{c} \quad \text { and } \quad \theta \theta^{c}=\epsilon_{l}^{1-(n+1) b} \mu^{-1} .
$$

Also set

$$
r^{\prime}=\operatorname{Ind}_{\operatorname{Gal}(\bar{F} / F M)}^{\operatorname{Gal}(\bar{F} / F)} \theta
$$

and $\bar{r}^{\prime}=r^{\prime} \bmod \lambda^{\prime}$.

Choose a lifting $\widetilde{\tau} \in \operatorname{Gal}(\bar{F} / F)$ of $\tau \in \operatorname{Gal}(F M / F)$ such that we have $\left(\epsilon_{l}^{1-(n+1) b} \mu^{-1}\right)(\widetilde{\tau})=1$. (This is possible as $M$ is linearly disjoint from the field $\bar{F}^{\text {ker } \epsilon_{l}^{1-(n+1) b} \mu^{-1}}$ over $\mathbb{Q}$, these extensions being ramified at disjoint sets of primes.)

Choose a non-zero primitive vector $e_{0}$ in $r^{\prime}$ such that

$$
r^{\prime}(\sigma) e_{0}=\theta(\sigma) e_{0}
$$

for all $\sigma \in \operatorname{Gal}(\bar{F} / F M)$, and set

$$
e_{i}=r^{\prime}\left(\widetilde{\tau}^{i}\right) e_{0}
$$

for $i=1, \ldots, 2 b-1$. Then for $\sigma \in \operatorname{Gal}(\bar{F} / M F)$ we have

$$
r^{\prime}(\sigma) e_{i}=\theta\left(\widetilde{\tau}^{-i} \sigma \widetilde{\tau}^{i}\right) e_{i}
$$

Moreover

$$
\begin{aligned}
r^{\prime}(\widetilde{\tau}) e_{2 b-1} & =\theta\left(\widetilde{\tau}^{n}\right) e_{0}=\left.\left(\theta \circ \operatorname{Art}_{F M}\right)\right|_{\mathbb{A}_{F M+}^{\times}}\left(\operatorname{Art}_{F M^{+}}^{-1} \widetilde{\tau}^{b}\right) \\
& =\left(\epsilon_{l}^{1-(n+1) b} \mu^{-1} \delta_{M}^{(1+\varepsilon) / 2}\right)\left(\widetilde{\tau}^{b}\right) e_{0}=-\varepsilon e_{0} .
\end{aligned}
$$

We define a perfect pairing on $r^{\prime}$ by

$$
\left\langle e_{i}, e_{j}\right\rangle^{\prime}= \begin{cases}1 & \text { if } j=i+n / 2 \\ -\varepsilon & \text { if } i=j+n / 2 \\ 0 & \text { otherwise }\end{cases}
$$

Then we see that

$$
\langle x, y\rangle^{\prime}=-\varepsilon\langle y, x\rangle^{\prime} \quad \text { and } \quad\left\langle r^{\prime}(\sigma) x, r^{\prime}(\sigma) y\right\rangle^{\prime}=\left(\epsilon_{l}^{1-(n+1) b} \mu^{-1}\right)(\sigma)\langle x, y\rangle^{\prime}
$$

for all $x, y \in \mathcal{O}_{M^{\prime}, \lambda^{\prime}}^{2 b}$ and $\sigma \in \operatorname{Gal}(\bar{F} / F)$. We also see that

$$
c \circ r^{\prime} \cong \mu \otimes r^{\prime}
$$


For $v \mid l$ the restriction $\left.r^{\prime}\right|_{\operatorname{Gal}\left(\bar{F}_{v} / F_{v}\right)}$ is crystalline and

$$
\begin{aligned}
\left.\bar{r}^{\prime}\right|_{\operatorname{Gal}\left(\bar{F}_{v} / F_{v}\right)} \cong & \omega_{2}^{1-(n+1) b} \oplus \omega_{2}^{2-(n+1) b-l} \oplus \cdots \oplus \omega_{2}^{-n b-(b-1) l} \\
& \oplus \omega_{2}^{l-(n+1) b l} \oplus \omega_{2}^{2 l-(n+1) b l-1} \oplus \cdots \oplus \omega_{2}^{-n b l-(b-1)} .
\end{aligned}
$$

Moreover for any $\tau^{\prime}: F_{v} \hookrightarrow \bar{M}_{\lambda^{\prime}}^{\prime}$ we have

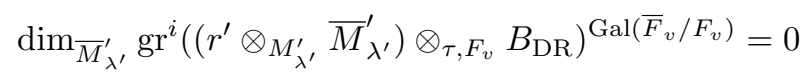

unless $0 \leq i \leq b-1$ or $n b \leq i \leq(n+1) b-1$ in which case

$$
\operatorname{dim}_{\bar{M}_{\lambda^{\prime}}^{\prime}} \operatorname{gr}^{i}\left(\left(r^{\prime} \otimes_{M_{\lambda^{\prime}}^{\prime}} \bar{M}_{\lambda^{\prime}}^{\prime}\right) \otimes_{\tau, F_{v}} B_{\mathrm{DR}}\right)^{\operatorname{Gal}\left(\bar{F}_{v} / F_{v}\right)}=1 .
$$

Now set $r^{\prime \prime}=r \otimes r^{\prime}$ and $\bar{r}^{\prime}=r^{\prime \prime} \bmod \lambda^{\prime}$. Thus

$$
r^{\prime \prime}: \operatorname{Gal}(\bar{F} / F) \rightarrow G S p_{2 b n}\left(\mathcal{O}_{M^{\prime}, \lambda^{\prime}}\right)
$$

and $r^{\prime \prime}$ has multiplier $\epsilon_{l}^{1-2 b n}$. Moreover $c \circ r^{\prime \prime} \cong r^{\prime \prime}$. Because the Brauer group of every finite extension of $\mathbb{F}_{l}$ is trivial we see that $\bar{r}^{\prime \prime}$ is conjugate to a homomorphism

$$
\operatorname{Gal}(\bar{F} / F) \rightarrow G L_{2 b n}\left(\mathbb{F}_{l}\right) .
$$

(Recall that $\operatorname{Gal}\left(\left(\mathcal{O}_{M^{\prime}} / \lambda^{\prime}\right) / \mathbb{F}_{l}\right) \cong\{1, c\}$.) Then by a lemma of Carayol (see Lemma 2.1 .10 of $[\mathrm{CHT}]$ ) we see that $r^{\prime \prime}$ can be replaced by a conjugate homomorphism

$$
r^{\prime \prime}: \operatorname{Gal}(\bar{F} / F) \rightarrow G L_{2 b n}\left(\mathbb{Z}_{l}\right) .
$$

Consider the vector subspace $V$ of $M_{n \times n}\left(\mathbb{Q}_{l}\right)$ consisting of anti-symmetric matrices $A$ such that

$$
r^{\prime \prime}(\sigma)^{t} A r^{\prime \prime}(\sigma)=\epsilon_{l}^{1-2 b n}(\sigma) A
$$

for all $\sigma \in \operatorname{Gal}(\bar{F} / F)$. The polynomial det does not vanish identically on $V \otimes_{\mathbb{Q}_{l}}$ $M_{\lambda^{\prime}}^{\prime}$ and so does not vanish identically on $V$. Thus we can find a non-degenerate alternating bilinear form $\langle,\rangle^{\prime \prime}$ on $\mathbb{Q}_{l}^{n}$ with

$$
\left\langle r^{\prime \prime}(\sigma) x, r^{\prime \prime}(\sigma) y\right\rangle^{\prime \prime}=\epsilon_{l}^{1-2 b n}(\sigma)\langle x, y\rangle^{\prime \prime}
$$

for all $x, y \in \mathbb{Q}_{l}^{n}$ and all $\sigma \in \operatorname{Gal}(\bar{F} / F)$.

For $v \mid l$ the restriction $\left.r^{\prime \prime}\right|_{\operatorname{Gal}\left(\bar{F}_{v} / F_{v}\right)}$ is crystalline and

$$
\left.\bar{r}^{\prime \prime}\right|_{\operatorname{Gal}\left(\bar{F}_{v} / F_{v}\right)} \cong \omega_{2}^{1-2 n b} \oplus \omega_{2}^{2-2 n b-l} \oplus \cdots \oplus \omega_{2}^{l(1-2 n b)} .
$$

Moreover

$$
\operatorname{dim}_{\mathbb{F}_{v}} \operatorname{gr}^{i}\left(r^{\prime \prime} \otimes_{\mathbb{Z}_{l}} B_{\mathrm{DR}}\right)^{\operatorname{Gal}\left(\bar{F}_{v} / F_{v}\right)}=0
$$


unless $0 \leq i \leq 2 b n-1$ in which case

$$
\operatorname{dim}_{\mathbb{F}_{v}} \operatorname{gr}^{i}\left(r^{\prime \prime} \otimes_{\mathbb{Z}_{l}} B_{\mathrm{DR}}\right)^{\operatorname{Gal}\left(\bar{F}_{v} / F_{v}\right)}=1 .
$$

Step 2: Automorphy of $r^{\prime \prime}$. As the primes that ramify in $\bar{F}^{\mathrm{ker} \bar{r}}\left(\zeta_{l}\right) / \mathbb{Q}$ and in $M / \mathbb{Q}$ are disjoint sets we may find $\sigma \in \operatorname{Gal}\left(\bar{F} / \bar{F}^{\text {ker ad } \bar{r}} M\right)$ so that $\epsilon_{l}(\sigma) \bmod l$ has order greater than $2 b$. Let

$$
\sigma^{\prime}=\sigma\left(\tau^{-1} \sigma \tau\right) \ldots\left(\tau^{1-2 b} \sigma \tau^{2 b-1}\right) .
$$

Then ad $\bar{r}^{\prime \prime}\left(\sigma^{\prime}\right)=1$ while $\epsilon_{l}\left(\sigma^{\prime}\right)=\epsilon_{l}(\sigma)^{2 b}$ is not equivalent to 1 modulo $l$. Write

$$
\operatorname{ad} \bar{r} \otimes_{\mathcal{O}_{M^{\prime}} / \lambda^{\prime}} \overline{\mathbb{F}}_{l}=V_{0}^{\oplus m_{0}} \oplus V_{1}^{\oplus m_{1}} \cdots \oplus V_{r}^{\oplus m_{r}}
$$

with $1=V_{0}, V_{1}, \ldots, V_{r}$ pairwise non-isomorphic, irreducible $\operatorname{Gal}\left(\bar{F} / F\left(\zeta_{l}\right)\right)$-modules and $1=m_{0}, m_{1}, \ldots, m_{r}$ positive integers. Write $\bar{\theta}$ for $\theta \bmod \lambda^{\prime}$. If $i, i^{\prime}=$ $1, \ldots, 2 b-1, i^{\prime \prime}=0, \ldots, 2 b-1$ and $\bar{\theta} / \bar{\theta}^{\tau^{i}}=\bar{\theta}^{i^{\prime \prime \prime}} / \bar{\theta}^{\tau^{i^{\prime}+i^{\prime \prime}}}$ on all inertia groups above $q$ then we see that either $i=i^{\prime}$ and $i^{\prime \prime}=0$, or $i+i^{\prime}=2 b$. Let $f_{0}, \ldots, f_{2 b-1}$ denote the basis of $\operatorname{Hom}\left(r^{\prime}, \mathcal{O}_{M^{\prime}, \lambda^{\prime}}\right)$ dual to $e_{0}, \ldots, e_{2 b-1}$. Then we can decompose

$$
\operatorname{ad} r^{\prime} \otimes_{\mathcal{O}_{M^{\prime}, \lambda^{\prime}}} \overline{\mathbb{F}}_{l}=\left(\bigoplus_{\chi \in \operatorname{Hom}\left(\operatorname{Gal}(M / \mathbb{Q}), \overline{\mathbb{F}}_{l}^{\times}\right)} W_{\chi}\right) \oplus\left(\bigoplus_{i=1}^{2 b-1} W_{i}\right),
$$

where

- $W_{\chi}$ is the span of $\sum_{i=0}^{2 b-1} \chi\left(\tau^{i}\right) e_{i} \otimes f_{i}$;

- $W_{i}$ is the span of $\left\{e_{j} \otimes f_{i+j}\right\}_{j=0, \ldots, 2 b-1}$.

Thus $W_{\chi} \cong \overline{\mathbb{F}}_{l}(\chi)$ and

Moreover

$$
W_{i} \cong \operatorname{Ind}_{\operatorname{Gal}(\bar{F} / M)}^{\operatorname{Gal}(\bar{Q})} \bar{\theta} / \bar{\theta}^{\tau^{i}}
$$

$$
\operatorname{ad} \bar{r}^{\prime \prime} \otimes \overline{\mathbb{F}}_{l} \cong\left(\bigoplus_{j=0}^{r} \bigoplus_{\chi \in \operatorname{Hom}\left(\operatorname{Gal}(M / \mathbb{Q}), \overline{\mathbb{F}}_{l}^{\times}\right)} V_{j}(\chi)^{m_{j}}\right) \oplus\left(\bigoplus_{j=0}^{r} \bigoplus_{i=1}^{2 b-1}\left(V_{j} \otimes W_{i}\right)^{m_{j}}\right) .
$$

Each factor $V_{j}(\chi)$ is irreducible over $F\left(\zeta_{l}\right)$ and (because $M F$ is linearly disjoint from $\bar{F}^{\operatorname{ker} \bar{r}}$ over $F$ ) there is no isomorphism $V_{j}(\chi) \cong V_{j^{\prime}}\left(\chi^{\prime}\right)$ unless $\chi=\chi^{\prime}$ and $j=j^{\prime}$. Moreover each factor

$$
V_{j} \otimes W_{i} \cong \operatorname{Ind}_{\operatorname{Gal}\left(\bar{F} / F M\left(\zeta_{l}\right)\right)}^{\left.\operatorname{Gal}\left(\overline{\zeta_{l}}\right)\right)}\left(V_{j} \otimes \bar{\theta} / \bar{\theta}^{\tau^{i}}\right)
$$


is irreducible, as otherwise

$$
V_{j} \cong V_{j}\left(\left(\overline{\theta \theta}^{\tau^{i+i^{\prime}}}\right) /\left(\bar{\theta}^{\tau^{i}} \bar{\theta}^{\tau^{i^{\prime}}}\right)\right)
$$

(which is impossible on inertia above $q$ ). Similarly

$$
\operatorname{Ind}_{\operatorname{Gal}\left(\bar{F} / F M\left(\zeta_{l}\right)\right)}^{\operatorname{Gal}\left(\bar{F} / F\left(\zeta_{l}\right)\right)}\left(V_{j} \otimes \bar{\theta} / \bar{\theta}^{\tau^{i}}\right) \cong \operatorname{Ind}_{\operatorname{Gal}\left(\bar{F} / F M\left(\zeta_{l}\right)\right)}^{\operatorname{Gal}\left(\bar{F} /\left(\zeta_{l}\right)\right)}\left(V_{j^{\prime}} \otimes \bar{\theta} / \bar{\theta}^{\tau^{i^{\prime}}}\right)
$$

if and only if $V_{j} \cong V_{j^{\prime}}$ and $i=i^{\prime}$ or $2 b-i^{\prime}$. Moreover looking at inertia above $q$ shows that

$$
V_{j}(\chi) \nRightarrow \operatorname{Ind}_{\operatorname{Gal}\left(\bar{F} / F M\left(\zeta_{l}\right)\right)}^{\operatorname{Gal}\left(\bar{F} /\left(\zeta_{l}\right)\right)}\left(V_{j^{\prime}} \otimes \bar{\theta} / \bar{\theta}^{\tau^{i}}\right)
$$

for all $i, j, j^{\prime}, \chi$.

Let $H$ (resp. $H^{\prime}$, resp. $\left.H^{\prime \prime}\right)$ denote the image $(\operatorname{ad} \bar{r}) \operatorname{Gal}\left(\bar{F} / F\left(\zeta_{l}\right)\right.$ ) (resp. $\left(\operatorname{ad} \bar{r}^{\prime}\right) \operatorname{Gal}\left(\bar{F} / F\left(\zeta_{l}\right)\right)$, resp. $\left.\left(\operatorname{ad} \bar{r}^{\prime \prime}\right) \operatorname{Gal}\left(\bar{F} / F\left(\zeta_{l}\right)\right)\right)$. We see that

$$
H^{0}\left(H^{\prime \prime}, \operatorname{ad}^{0} \bar{r}^{\prime \prime}\right)=(0) \text {. }
$$

Note that the only scalar element of $H$ or $H^{\prime}$ (thought of as subgroups of Aut( $\left.\operatorname{ad} \bar{r}\right)$ and $\left.\operatorname{Aut}\left(\operatorname{ad} \bar{r}^{\prime}\right)\right)$ is the identity and so

$$
H \times H^{\prime} \hookrightarrow \operatorname{Aut}\left(\operatorname{ad} \bar{r}^{\prime \prime}\right) .
$$

Thus there is a finite group $\bar{H}$ and surjections $\pi: H \rightarrow \bar{H}$ and $\pi^{\prime}: H^{\prime} \rightarrow \bar{H}$ so that $H^{\prime \prime}$ is the set of elements $\left(h, h^{\prime}\right) \in H \times H^{\prime}$ such that $\pi(h)=\pi^{\prime}\left(h^{\prime}\right)$. Let $K$ (resp. $K^{\prime}$ ) denote the kernel of $\pi$ (resp. $\pi^{\prime}$ ). Note that the image of the inertia group at any prime above $q$ in $H^{\prime}$ is contained in $K^{\prime}$ (as $\bar{r}$ is unramified above $q$ ). Also note that the restriction of $H^{\prime} \rightarrow \operatorname{Gal}\left(M F\left(\zeta_{l}\right) / F\left(\zeta_{l}\right)\right)$ to $K^{\prime}$ is surjective (as $M$ is linearly disjoint from $\bar{F}^{\text {ker } \bar{r}}\left(\zeta_{l}\right)$ over $\mathbb{Q}$, these fields being ramified at disjoint sets of primes). Thus

$$
\left(\operatorname{ad} \bar{r}^{\prime \prime}\right)^{K^{\prime}}=\left(\operatorname{ad} \bar{r}^{\prime}\right)^{K^{\prime}} \otimes(\operatorname{ad} \bar{r})=\operatorname{ad} \bar{r}
$$

and we see that

$$
(0)=H^{1}\left(H, \operatorname{ad}^{0} \bar{r}\right)=H^{1}\left(H^{\prime \prime} / K^{\prime},\left(\operatorname{ad}^{0} \bar{r}^{\prime \prime}\right)^{K^{\prime}}\right) \stackrel{\sim}{\rightarrow} H^{1}\left(H^{\prime \prime}, \operatorname{ad}^{0} \bar{r}^{\prime \prime}\right)
$$

(because $l \nmid \# K^{\prime}$ implies that $\left.H^{1}\left(K^{\prime}, \operatorname{ad}^{0} \bar{r}^{\prime \prime}\right)=(0)\right)$.

Fix a copy $V_{j} \subset \operatorname{ad} \bar{r} \otimes \overline{\mathbb{F}}_{l}$. We can find a $\sigma \in \operatorname{Gal}\left(\bar{F} / M F\left(\zeta_{l}\right)\right)$ and a simple root $\alpha$ of the characteristic polynomial of $\bar{r}(\sigma)$ such that $\pi_{\bar{r}(\sigma), \alpha} V_{j} i_{\bar{r}(\sigma), \alpha} \neq(0)$. Altering $\sigma$ by elements of inertia subgroups at primes above $q$ we may further suppose that for $i=1, \ldots, 2 b-1$ the ratio $\left(\bar{\theta} / \bar{\theta}^{\tau^{i}}\right)(\sigma)$ does not equal $\alpha^{\prime} / \alpha$ for any root $\alpha^{\prime}$ (including $\alpha$ ) of the characteristic polynomial of $\bar{r}(\sigma)$. (Here we use the 
fact that $q>2 n$ to see that we can choose an element $\varsigma$ of the inertia group at $\tau^{-i} \mathfrak{q}$ so that $\bar{\theta}^{\tau^{i}}(\varsigma)$ avoids $n$ values and $\bar{\theta}^{\tau^{b+i}}(\varsigma)$ also avoids $n$ values.) Then $\alpha \bar{\theta}(\sigma)$ is a simple root of the characteristic polynomial of $\bar{r}^{\prime \prime}(\sigma)$ and

$$
\begin{aligned}
\pi_{\bar{r}^{\prime \prime}(\sigma), \alpha \bar{\theta}(\sigma)} & V_{j}(\chi) i_{\bar{r}^{\prime \prime}(\sigma), \alpha \bar{\theta}(\sigma)} \\
& =\left(\pi_{\bar{r}(\sigma), \alpha} V_{j} i_{\bar{r}(\sigma), \alpha}\right)\left(\pi_{\bar{r}^{\prime}(\sigma), \bar{\theta}(\sigma)}\left(\sum_{j=0}^{2 b-1} \chi\left(\tau^{j}\right) e_{j} \otimes f_{j}\right) i_{\bar{r}^{\prime}(\sigma), \bar{\theta}(\sigma)}\right) \\
& =\pi_{\bar{r}(\sigma), \alpha} V_{j} i_{\bar{r}(\sigma), \alpha} \neq(0)
\end{aligned}
$$

for every $\chi \in \operatorname{Hom}\left(\operatorname{Gal}(M / \mathbb{Q}), \overline{\mathbb{F}}_{l}^{\times}\right)$.

Now fix indices $j=0, \ldots, r$ and $i=1, \ldots, b$. Also fix $\gamma: W_{i} \stackrel{\sim}{\rightarrow} W_{2 b-i}$ (unless $i=b)$ such that $\left.\gamma\left(e_{0} \otimes f_{i}\right)=e_{b+i} \otimes f_{b}\right)$. Any submodule of ad $\bar{r}^{\prime \prime} \otimes \overline{\mathbb{F}}_{l}$ which is isomorphic to $V_{j} \otimes W_{i}$ is of the form

$$
\left\{\eta_{1}(v) \otimes w+\eta_{2}(v) \otimes \gamma(w): v \in V_{j} \text { and } w \in W_{i}\right\}
$$

where $\eta_{1}$ and $\eta_{2}$ are embeddings $V_{j} \hookrightarrow \operatorname{ad} \bar{r} \otimes \overline{\mathbb{F}}_{l}$. (If $i=b$ we suppress the second term.) Choose $\sigma \in \operatorname{Gal}\left(\bar{F} / F\left(\zeta_{l}\right)\right)$ and a simple root $\alpha$ of the characteristic polynomial of $\bar{r}(\sigma)$ such that

- $\sigma$ maps to the generator $\tau$ of $\operatorname{Gal}\left(M F\left(\zeta_{l}\right) / F\left(\zeta_{l}\right)\right)$;

- $\pi_{\bar{r}(\sigma), \alpha} \eta_{1}\left(V_{j}\right) i_{\bar{r}(\sigma), \alpha} \neq(0)$;

- no other root of the characteristic polynomial of $\bar{r}(\alpha)$ has $(2 b)^{\text {th }}$ power equal to $\alpha^{2 b}$.

(Here we are using the assumption that $\bar{r}\left(\operatorname{Gal}\left(\bar{F} / F\left(\zeta_{l}\right)\right)\right)$ is $2 b$-big.) Write

$$
\bar{r}^{\prime}(\sigma) e_{i}=\beta_{i} e_{i+1}
$$

for $i=0, \ldots, 2 b-1$ (where we take $i+1$ modulo $2 b$ ). The roots of the characteristic polynomial of $\bar{r}^{\prime}(\sigma)$ are exactly the $(2 b)^{\text {th }}$ roots of $\beta_{0} \ldots \beta_{2 b-1}$. If $\beta^{2 b}=\beta_{0} \ldots \beta_{2 b-1}$ then a corresponding eigenvector is

$$
e_{0}+\frac{\beta_{0}}{\beta} e_{1}+\frac{\beta_{0} \beta_{1}}{\beta^{2}} e_{2}+\cdots+\frac{\beta_{0} \ldots \beta_{2 b-2}}{\beta^{2 b-1}} e_{2 b-1}
$$

and

$$
\pi_{\bar{r}^{\prime}(\sigma), \beta} e_{j}=\frac{\beta^{j}}{2 b \beta_{0} \beta_{1} \ldots \beta_{j-1}}\left(e_{0}+\frac{\beta_{0}}{\beta} e_{1}+\cdots+\frac{\beta_{0} \ldots \beta_{2 b-2}}{\beta^{2 b-1}} e_{2 b-1}\right) .
$$


Moreover $\alpha \beta$ is a simple root of the characteristic polynomial of $\bar{r}^{\prime \prime}(\sigma)$ and

$$
\begin{aligned}
\pi_{\bar{r}^{\prime \prime}(\sigma), \alpha \beta}( & \left.\eta_{1}(v) \otimes e_{0} \otimes f_{i}+\eta_{2}(v) \otimes e_{b+i} \otimes f_{b}\right) i_{\bar{r}^{\prime \prime}(\sigma), \alpha \beta} \\
= & \left(\pi_{\bar{r}(\sigma), \alpha} \eta_{1}(v) i_{\bar{r}(\sigma), \alpha}\right)\left(\pi_{\bar{r}^{\prime}(\sigma), \beta} e_{0} \otimes f_{i} i_{\bar{r}^{\prime}(\sigma), \beta}\right) \\
& +\left(\pi_{\bar{r}(\sigma), \alpha} \eta_{2}(v) i_{\bar{r}(\sigma), \alpha}\right)\left(\pi_{\bar{r}^{\prime}(\sigma), \beta} e_{b+i} \otimes f_{b} i_{\bar{r}^{\prime}(\sigma), \beta}\right) \\
= & \frac{1}{2 b \beta^{i}}\left(\frac{\beta_{0} \ldots \beta_{i-1} \pi_{\bar{r}(\sigma), \alpha} \eta_{1}(v) i_{\bar{r}(\sigma), \alpha}+\beta^{2 i}}{\beta_{b} \ldots \beta_{b+i-1}} \pi_{\bar{r}(\sigma), \alpha} \eta_{2}(v) i_{\bar{r}(\sigma), \alpha}\right),
\end{aligned}
$$

where again we drop the second term if $i=b$. For some choice of $v$ and $\beta$ this will be non-zero.

We conclude that $\bar{r}^{\prime \prime}(\operatorname{Gal}(\bar{F} / F))$ is big. Theorem 6.4 then tells us that there is a Galois totally real extension $F^{\prime} / F$, a RAESDC automorphic representation $\Pi$ of $G L_{2 b n}\left(\mathbb{A}_{F^{\prime}}\right)$ of weight 0 and an isomorphism $\imath: \overline{M_{\lambda^{\prime}}^{\prime}} \stackrel{\sim}{\rightarrow} \mathbb{C}$ such that $r_{l, \imath}(\Pi) \cong r^{\prime \prime}$. (Note that $l \equiv-1 \bmod N$ and that $N$ is not divisible by any primes at which $r^{\prime \prime}$ ramifies.)

Step 3: Automorphy of $r$. We see that

$$
\Pi^{\vee} \cong \Pi \text { and } \Pi \otimes\left(\kappa \circ \operatorname{Art}_{F} \circ \text { det }\right) \cong \Pi
$$

We will claim that there is a cuspidal automorphic representation $\Pi_{M^{+}}$of $G L_{2 n}\left(\mathbb{A}_{F M^{+}}\right)$such that

$$
\Pi_{M^{+}} \otimes\left(\delta_{M} \circ \operatorname{Art}_{F M^{+}} \circ \text { det }\right) \cong \Pi_{M^{+}},
$$

such that $\Pi_{M^{+}, v}$ is regular for all $v \mid \infty$ and such that the base change from $F$ to $F M^{+}$of $\Pi$ is equivalent to

$$
\Pi_{M^{+}} \boxplus \Pi_{M^{+}}^{\tau} \boxplus \cdots \boxplus \Pi_{M^{+}}^{\tau^{b-1}}
$$

in the sense that for all $v$ the base change from $F_{\left.v\right|_{F}}$ to $\left(F M^{+}\right)_{v}$ of $\Pi_{\left.v\right|_{F}}$ is

$$
\Pi_{M^{+}, v} \boxplus\left(\Pi_{M^{+}}^{\tau}\right)_{v} \boxplus \cdots \boxplus\left(\Pi_{M^{+}}^{\tau^{b-1}}\right)_{v} .
$$

This can be proved for all intermediate fields $M^{+} \supset M_{1} \supset \mathbb{Q}$ by induction on $\left[M_{1}: \mathbb{Q}\right]$. So suppose that $M+\supset M_{2} \supset M_{1} \supset \mathbb{Q}$ with $M_{2} / M_{1}$ cyclic of prime degree and suppose that we have already found a cuspidal automorphic representation $\Pi_{M_{1}}$ of $G L_{2 n\left[M^{+}: M_{1}\right]}\left(\mathbb{A}_{F M_{1}}\right)$ with the corresponding properties. Then

$$
\Pi_{M_{1}} \otimes\left(\kappa \otimes \operatorname{Art}_{F M_{1}} \otimes \operatorname{det}\right) \cong \Pi_{M_{1}}^{\tau^{i}}
$$

for some $i=0, \ldots,\left[M^{+}: M_{1}\right]-1$. If $i>0$ then for $v \mid \infty$,

$$
\Pi_{M_{1}, v} \boxplus\left(\Pi_{M_{1}}^{\tau}\right)_{v} \boxplus \cdots \boxplus\left(\Pi_{M_{1}}^{\tau^{\left[M_{1}: \mathbb{Q}\right]-1}}\right)_{v}
$$


is not regular, so that $\Pi_{\left.v\right|_{F}}$ is not regular, a contradiction. Thus

$$
\Pi_{M_{1}} \otimes\left(\kappa \otimes \operatorname{Art}_{F M_{1}} \otimes \text { det }\right) \cong \Pi_{M_{1}}
$$

and the result for $M_{2}$ follows from Theorem 4.2 of $\mathrm{AC}$.

We can further conclude (using Theorem 4.2 of $[\mathrm{AC}$ ) that there is a cuspidal automorphic representation $\pi$ of $G L_{n}\left(\mathbb{A}_{F M}\right)$ such that

- the base change of $\Pi$ to $M F$ is equivalent to

$$
\pi \boxplus \pi^{\tau} \boxplus \cdots \boxplus \pi^{\tau^{2 b-1}} ;
$$

- the representations $\pi^{\tau^{i}}$ for $i=0, \ldots, 2 b-1$ are pairwise non-isomorphic;

- $\pi \otimes|\operatorname{det}|^{n(1 / 2-b)}$ is regular algebraic;

- $\pi^{\vee} \cong \pi^{c}$.

For the second assertion we use the regularity of $\Pi_{v}$ for $v \mid \infty$. For the last of these assertions note that $\pi^{\vee} \cong \pi^{\tau^{i}}$ for some $i=0, \ldots, 2 b-1$. As $\left(\pi^{\vee}\right)^{\vee} \cong \pi$ we deduce that either $i=0$ or $n / 2$. So we must rule out the case $\pi^{\vee} \cong \pi$. If this held then $\pi$ would have a unitary central character and so would itself be unitary. On the other hand for $v \mid \infty$ the Harish-Chandra parameter of $\pi_{v}$ lies in $(1 / 2) X^{*}\left(\widetilde{T} \times_{\mathbb{R}} \mathbb{C}\right)$, and so by Lemma 7.1 we have $\pi_{v}^{c} \cong \pi_{v}^{\vee} \cong \pi_{v}$. Again this would contradict the regularity of $\pi_{v} \oplus \pi_{v}^{c}$ and $\Pi_{M^{+},\left.v\right|_{F M^{+}}}$.

Thus $\pi \otimes|\operatorname{det}|^{n(1 / 2-b)}$ is a RAECSDC representation of $G L_{n}\left(\mathbb{A}_{F M}\right)$ and we can associate an $l$-adic representation $r_{l, \imath}(\pi)$ to it. Then

$$
\begin{aligned}
\left.r_{l, \imath}(\Pi)\right|_{\mathrm{Gal}(\bar{F} / F M)} & \cong r_{l, \imath}\left(\pi \otimes|\operatorname{det}|^{n(1 / 2-b)}\right) \oplus r_{l, \imath}\left(\pi \otimes|\operatorname{det}|^{n(1 / 2-b)}\right)^{\tau} \\
& \oplus \cdots \oplus r_{l, \imath}\left(\pi \otimes|\operatorname{det}|^{n(1 / 2-b)}\right)^{\tau^{2 b-1}} \\
\cong & \left(\left.r\right|_{\operatorname{Gal}(\bar{F} / F M)} \otimes \theta\right) \oplus\left(\left.r\right|_{\operatorname{Gal}(\bar{F} / F M)} \otimes \theta^{\tau}\right) \oplus \cdots \oplus\left(\left.r\right|_{\operatorname{Gal}(\bar{F} / F M)} \otimes \theta^{\tau^{2 b-1}}\right) .
\end{aligned}
$$

Hence

$$
\left.r\right|_{\mathrm{Gal}(\bar{F} / F M)} \cong r_{l, \imath}\left(\pi \otimes|\operatorname{det}|^{n(1 / 2-b)}\right) \otimes \theta^{-\tau^{j}}
$$

for some $j$ and $\left.r\right|_{\operatorname{Gal}(\bar{F} / F M)}$ is automorphic, and we conclude that $r$ is automorphic.

\section{$\S 8$. Applications}

Let $F$ be a totally real field. We will call a RAESDC automorphic representation $\pi$ of $G L_{2}\left(\mathbb{A}_{F}\right) C M$ if there is a non-trivial character $\chi: \mathbb{A}_{F}^{\times} / F^{\times} \rightarrow \mathbb{C}^{\times}$with $\pi \cong \pi \otimes(\chi \circ$ det $)$. In this case $\chi^{2}=1$ and $\pi$ is the automorphic induction of a grossencharacter of the quadratic extension defined by $\chi$. 
Theorem 8.1. Suppose that $\pi$ is a RAESDC automorphic representation of $G L_{2}\left(\mathbb{A}_{\mathbb{Q}}\right)$ and that $m \in \mathbb{Z}_{>0}$. If $\pi$ is not $C M$ then there is a Galois totally real number field $F / \mathbb{Q}$ and a RAESDC automorphic representation $S_{F}^{m}(\pi)$ of $G L_{m+1}\left(\mathbb{A}_{F}\right)$ such that for all primes $l$ and all $\imath: \overline{\mathbb{Q}}_{l} \stackrel{\sim}{\rightarrow} \mathbb{C}$ we have

$$
r_{l, i}\left(S_{F}^{m}(\pi)\right)=\left.\operatorname{Symm}^{m} r_{l, \imath}(\pi)\right|_{\operatorname{Gal}(\overline{\mathbb{Q}} / F)} .
$$

Proof. We may suppose that $\pi_{\infty}$ has weight $(k-2,0)$ for some $k \in \mathbb{Z}_{>1}$. Let $N \subset \mathbb{C}$ denote the field of coefficients of $\pi$ (i.e. the extension of $\mathbb{Q}$ generated by the eigenvalues of the Hecke operator

$$
G L_{2}\left(\mathbb{Z}_{p}\right)\left(\begin{array}{ll}
p & 0 \\
0 & 1
\end{array}\right) G L_{2}\left(\mathbb{Z}_{p}\right)
$$

on $\pi_{p}^{G L_{2}\left(\mathbb{Z}_{p}\right)}$ for all $p$ for which $\pi_{p}$ is unramified). (Strictly speaking: the Hecke operator coming from the product of the characteristic function of this double coset and the Haar measure on $G L_{2}\left(\mathbb{Q}_{p}\right)$ giving $G L_{2}\left(\mathbb{Z}_{p}\right)$ volume 1.) Then $N$ is a CM field. There is a continuous character

$$
\nu: \operatorname{Gal}(\overline{\mathbb{Q}} / \mathbb{Q}) \rightarrow N^{\times}
$$

such that for all $l$ and $\imath$ we have

$$
\operatorname{det} r_{l, \imath}=\epsilon_{l}^{1-k}\left(l^{-1} \circ \nu\right) .
$$

(The character $\nu$ is sometimes called the nebentypus character. We have $c \circ \pi \cong$ $\pi \otimes\left(\nu \circ \mathrm{Art}_{\mathbb{Q}} \circ \mathrm{det}\right)^{-1}$.) Let $M^{\prime}$ be the CM field which Theorem 7.6 asserts exist for $n=m+1$ and $b=k-1$ and $\mu=\nu^{m}$. Let $N^{\prime}$ denote the normal closure of $N M^{\prime}$ over $\mathbb{Q}$.

By a theorem of Ribet (see Theorem 2.1 of $[\mathrm{R}$ and the first three lines of its proof) for all but finitely many $l$ the image $\bar{r}_{l, l}(\operatorname{Gal}(\overline{\mathbb{Q}} / \mathbb{Q}))$ contains $S L_{2}\left(\mathbb{F}_{l}\right)$. Moreover for all but finitely many $l$ either $r_{l, \imath}(\pi)$ is ordinary of weight $(k-2,0)$ for all $\imath$ or

$$
\left.\bar{r}_{l, 2}\right|_{\mathbb{Q}_{l}} \cong \omega_{2}^{1-k} \oplus \omega_{2}^{l(1-k)}
$$

for all $\imath$. In the former case we call $l$ ordinary, in the latter case we call $l$ supersingular.

Thus for all but finitely many ordinary primes $l$ Theorem 7.5 tells us that there exists a Galois totally real field $F / \mathbb{Q}$ such that $\left.\operatorname{Symm}^{m} r_{l, l}\right|_{\mathrm{Gal}(\overline{\mathbb{Q}} / F)}$ is automorphic of weight $(m(k-2), \ldots, k-2,0)_{F}$. For all but finitely many supersingular primes for which $\left[\mathrm{Frob}_{l}\right]=[c] \subset \operatorname{Gal}\left(N^{\prime} / \mathbb{Q}\right)$ Theorem 7.6 tells us that there exists a Galois

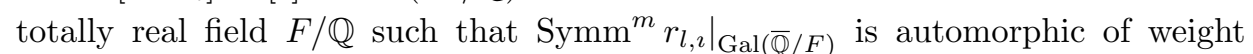
$(m(k-2), \ldots,(k-2), 0)_{F}$. Thus, for all but finitely many of the infinitely many 
primes $l$ with $\left[\operatorname{Frob}_{l}\right]=[c] \subset \operatorname{Gal}\left(N^{\prime} / \mathbb{Q}\right)$, there exists a Galois totally real field $F / \mathbb{Q}$ such that $\left.\operatorname{Symm}^{m} r_{l, \imath}\right|_{\mathrm{Gal}(\overline{\mathbb{Q}} / F)}$ is automorphic of weight $(m(k-2), \ldots,(k-2), 0)_{F}$. The theorem follows.

At a referee's request we remark that for any finite subset $\mathcal{M} \subset \mathbb{Z}_{>0}$ we can find a Galois totally real field $F / \mathbb{Q}$ such that $\left.\operatorname{Symm}^{m} r_{l, \imath}(\pi)\right|_{\mathrm{Gal}(\overline{\mathbb{Q}} / F)}$ is automorphic for all $m \in \mathcal{M}$. To see this one must check that Theorems 7.5 and 7.6 can be modified to simultaneously realize the automorphy of a finite number of representations $r_{1}, \ldots, r_{s}$ of $\operatorname{Gal}(\bar{F} / F)$ (corresponding to a finite number of characters $\mu_{1}, \ldots, \mu_{s}$ ) over one Galois totally real extension $F^{\prime} / F$. (In the case of Theorem 7.6 the set of primes at which it will apply will depend on the $\left\{\mu_{i}\right\}$.) The generalized form of these theorems would in turn rely on slight generalizations of Theorems 6.3 and 6.4 which would simultaneously realize the automorphy of a finite number of representations $r_{1}, \ldots, r_{s}$ of $\operatorname{Gal}(\bar{F} / F)$ over one Galois totally real extension $F^{\prime} / F$. To prove these latter generalizations one simply chooses $F^{\prime}$ so that the schemes $T_{\bar{r}_{i} \times \bar{r}_{i}^{\prime}}$ simultaneously have $F^{\prime}$ rational points of the desired sort. We leave it to the interested reader to provide the details.

Corollary 8.2. Suppose that $\pi$ is a non-CM RAESDC automorphic representation of $G L_{2}\left(\mathbb{A}_{\mathbb{Q}}\right)$ of weight $(k-2,0)$ with $k \in \mathbb{Z}_{>1}$. Suppose also that $m \in \mathbb{Z}_{\geq 0}$ and that $\psi: \mathbb{A}^{\times} / \mathbb{Q}^{\times} \mathbb{R}_{>0}^{\times} \rightarrow \mathbb{C}^{\times}$is a continuous character. Then there is a meromorphic function $L\left(S^{m}(\pi) \times \psi, s\right)$ on the whole complex plane such that for any prime $l$ and any isomorphism $\imath: \overline{\mathbb{Q}}_{l} \stackrel{\sim}{\rightarrow} \mathbb{C}$ we have $L\left(S^{m}(\pi) \times \psi, s\right)=L\left(\imath\left(\operatorname{Symm}^{m} r_{l, \imath}(\pi)\right) \otimes\right.$ $\left.r_{l, \imath}(\psi), s\right)$. The expected functional equation holds between $L\left(S^{m}(\pi) \times \psi, s\right)$ and $L\left(S^{m}\left(\pi^{\vee} \otimes|\operatorname{det}|^{2-k}\right) \times \psi^{-1}, 1+m(k-1)-s\right)$. Moreover if $m>0$ or $\psi \neq 1$ then $L\left(S^{m}(\pi) \times \psi, s\right)$ is holomorphic and non-zero in $\operatorname{Re} s \geq 1+m(k-1) / 2$.

Proof. This follows from the previous theorem as in the proof of 4.2 of [HSBT].

We now formulate the Sato-Tate conjecture for RAESDC automorphic representations of $G L_{2}\left(\mathbb{A}_{\mathbb{Q}}\right)$ following Gee. For $a \in \mathbb{Z}_{>0}$ we will let $U(2)_{a}$ denote the subgroup of $U(2)$ consisting of matrices $g$ with $(\operatorname{det} g)^{a}=1$. We will let $U(2)_{a} / \sim$ denote the space of conjugacy classes of $U(2)_{a}$. By Haar measure on $U(2)_{a} / \sim$ we will mean the push-forward of the Haar measure on $U(2)_{a}$ with total volume 1.

Corollary 8.3. Suppose that $\pi$ is a non-CM RAESDC automorphic representation of $G L_{2}\left(\mathbb{A}_{\mathbb{Q}}\right)$ of weight $(k-2,0)$ with $k \in \mathbb{Z}_{>1}$. Let $\psi$ denote the product of the central character of $\pi$ with $|\cdot|^{k-2}$. The character $\psi$ has finite order and we denote this order $a$. For all but finitely many primes $p$ the component $\pi_{p}$ will be unramified and we will let $\left[F_{p}\right]$ denote the conjugacy class of the matrix $p^{1-k / 2} \operatorname{rec}_{\mathbb{Q}_{p}}\left(\pi_{p}\right)\left(\right.$ Frob $\left._{p}\right)$, which lies in $U(2)_{a}$ (and has determinant $\left.\psi_{p}(p)\right)$. 
The conjugacy classes $\left[F_{p}\right]$ are equidistributed in $U(2)_{a} / \sim$ with respect to Haar measure.

Proof. This follows from the previous corollary and the corollary to Theorem 2 of [Se1, as explained on page I-26 of [Se1]. (We remark that the irreducible representations of $U(2)_{a}$ are the representations $\operatorname{det}^{n} \otimes \operatorname{Symm}^{m}$ for $n=0, \ldots, a-1$ and $m \in \mathbb{Z}_{\geq 0}$.)

This corollary can be reformulated as follows.

Corollary 8.4. Suppose that $\pi$ is a non-CM RAESDC automorphic representation of $G L_{2}\left(\mathbb{A}_{\mathbb{Q}}\right)$ of weight $(k-2,0)$ with $k \in \mathbb{Z}_{>1}$. Let $\psi$ denote the product of the central character of $\pi$ with $|\cdot|^{k-2}$, so that $\psi$ has finite order. Let $\zeta$ be a root of unity such that $\zeta^{2}$ lies in the image of $\psi$. For all but finitely many $p$ the space $\pi_{p}^{G L_{2}\left(\mathbb{Z}_{p}\right)}$ is one-dimensional. Let $t_{p}$ denote the eigenvalue of the Hecke operator

$$
\left[G L_{2}\left(\mathbb{Z}_{p}\right)\left(\begin{array}{ll}
p & 0 \\
0 & 1
\end{array}\right) G L_{2}\left(\mathbb{Z}_{p}\right)\right]
$$

on $\pi_{p}^{G L_{2}\left(\mathbb{Z}_{p}\right)}$ (or strictly speaking, of the Hecke operator coming from the product of the characteristic function of this double coset with the Haar measure on $G L_{2}\left(\mathbb{Q}_{p}\right)$ which gives $G L_{2}\left(\mathbb{Z}_{p}\right)$ volume 1$)$. If $\psi_{p}(p)=\zeta^{2}$ then $t_{p} /\left(2 p^{(k-1) / 2} \zeta\right) \in[-1,1] \subset \mathbb{R}$.

As $p$ varies over primes with $\psi_{p}(p)=\zeta^{2}$, the numbers $t_{p} /\left(2 p^{(k-1) / 2} \zeta\right)$ are equidistributed in $[-1,1]$ with respect to the measure $(2 / \pi) \sqrt{1-t^{2}} d t$.

As special cases we mention the following corollaries, though many similar examples are also available.

Corollary 8.5. Write

$$
\sum_{n=1}^{\infty} \tau(n) q^{n}=q \prod_{n}\left(1-q^{n}\right)^{24}
$$

i.e. $\tau(n)$ denotes Ramanujan's $\tau$ function. Then the numbers $\tau(p) /\left(2 p^{11 / 2}\right)$ are equidistributed in $[-1,1]$ with respect to the measure $(2 / \pi) \sqrt{1-t^{2}} d t$.

Corollary 8.6. Let $N_{12}(n)$ denote the number of elements of $\mathbb{Z}^{12}$ with Euclidean norm $\sqrt{n}$, i.e. the number of ways $n$ can be written as the sum of 12 perfect squares (where the order matters). As $p$ runs over prime numbers,

$$
\left(N_{12}(p)-8\left(p^{5}+1\right)\right) /\left(32 p^{5 / 2}\right)
$$

lies in $[-1,1]$ and these numbers are equidistributed in $[-1,1]$ with respect to the measure $(2 / \pi) \sqrt{1-t^{2}} d t$. 
Proof. For an odd prime $p$ we have

$$
N_{12}(p)=8\left(p^{5}+1\right)+16 a_{p},
$$

where $a_{p}$ is the coefficient of $q^{p}$ in the cuspidal newform

$$
q \prod_{n=1}^{\infty}\left(1-q^{2 n}\right)^{12}
$$

of weight 6 on $\Gamma_{0}(4)$. (See Gl].)

The following results do not depend on the innovations of this paper (as far as there are any innovations in this paper). However the results of [Sh] and CHLN] have made them accessible, so we are taking the opportunity to record them in print. We will call an elliptic curve $E$ over a number field $F C M$ if $\operatorname{End}(E / \bar{F}) \neq \mathbb{Z}$.

Theorem 8.7. Suppose that $F$ is a totally real field, that $E / F$ is an elliptic curve and that $m \in \mathbb{Z}_{>0}$. If $E$ is not $C M$ then there is a Galois totally real field $F^{\prime} / F$ and a RAESDC automorphic representation $S_{F^{\prime}}^{m}(E)$ of $G L_{m+1}\left(\mathbb{A}_{F^{\prime}}\right)$ such that for all primes $l$ and all $\imath: \overline{\mathbb{Q}}_{l} \stackrel{\sim}{\rightarrow} \mathbb{C}$ we have

$$
r_{l, \imath}\left(S_{F^{\prime}}^{m}(E)\right)=\operatorname{Symm}^{m} H_{\mathrm{et}}^{1}\left(E \times \operatorname{Spec} \bar{F}, \overline{\mathbb{Q}}_{l}\right) .
$$

Proof. Let $r_{E, l}$ denote the representation of $\operatorname{Gal}(\bar{F} / F)$ which occurs on $H_{\text {et }}^{1}(E \times$ Spec $\left.\bar{F}, \mathbb{Z}_{l}\right)$. As $E$ is not CM the image of $r_{E, l}$ is $G L_{2}\left(\mathbb{Z}_{l}\right)$ for all but finitely many primes $l$. (See $[\mathrm{Se} 2$.) Thus there is a set $S$ of primes of $F$ with Dirichlet density zero such that for $v \notin S$ the elliptic curve $E$ has good ordinary reduction at $v$ and $F_{v} \cong \mathbb{Q}_{\left.v\right|_{\mathbb{Q}}}$. (Take $S$ to consist of the set of primes of $F$ where $E$ has bad reduction, where $F_{v} \neq \mathbb{Q}_{\left.v\right|_{\mathbb{Q}}}$, where $\# E(k(v))=1+\# k(v)$, or which divide 6 . Note that for $v \nmid l$ a prime of good reduction of $E$ the latter is equivalent to $\operatorname{tr} r_{E, l}\left(\operatorname{Frob}_{v}\right)=0$ and the set of matrices of trace zero in $G L_{2}\left(\mathbb{Z}_{l}\right)$ has Haar measure 0.) Choose a rational prime $l$ so that no place of $F$ above $l$ lies in $S$ and so that $r_{E, l}(\operatorname{Gal}(\bar{F} / F))=G L_{2}\left(\mathbb{Z}_{l}\right)$. Then apply Theorem 6.3 to Symm ${ }^{m} r_{E, l}$ and the theorem follows.

Corollary 8.8. Suppose that $F$ is a totally real number field, that $E / F$ is an elliptic curve and that $m \in \mathbb{Z}_{>0}$. The L-function $L\left(\operatorname{Symm}^{m} E, s\right)$ has meromorphic continuation to $\mathbb{C}$ and satisfies the expected functional equation relating the values at $s$ and $m+1-s$. If $E$ is not $C M$ then $L\left(\mathrm{Symm}^{m} E, s\right)$ is holomorphic and non-zero in $\operatorname{Re} s \geq 1+m / 2$.

Proof. If $E$ is $\mathrm{CM}$ then the result is well known. If $E$ is not CM it follows from the previous theorem as in the proof of 4.2 of [HSBT]. 
Corollary 8.9. If $F$ is a totally real field and if $E / F$ is a non-CM elliptic curve then the numbers

$$
(1+\# k(v)-\# E(k(v))) /(2 \sqrt{\# k(v)})
$$

are equidistributed in $[-1,1]$ with respect to the measure $(2 / \pi) \sqrt{1-t^{2}} d t$.

Proof. This follows from the previous corollary and the corollary to Theorem 2 of Se1], as explained on page I-26 of [Se1].

\section{Acknowledgements}

M.H. wishes to thank Magdalen College and the Mathematical Institute of Oxford for providing extraordinary living and working conditions during the preparation of this paper. We would all like to thank Jean-Pierre Labesse for revising his paper La at short notice so that it covered exactly the results (Corollary 5.3 and Theorem 5.4) we needed. Finally we would like to thank the referees for their helpful comments.

The research of R. Taylor was partially supported by NSF Grant DMS0600716.

\section{References}

[AC] J. Arthur and L. Clozel, Simple algebras, base change and the advanced theory of the trace formula, Ann. of Math. Stud. 120, Princeton Univ. Press, 1989. Zbl 0682.10022 MR 1007299

[BL1] T. Barnet-Lamb, Potential automorphy for certain Galois representations to $G L_{2 n}$, arXiv:0811.158

[BL2] _ On the potential automorphy of certain odd-dimensional Galois representations, Compos. Math. 146 (2010), 607-620. Zbl pre05722159 MR 2644929

[BLGG] T. Barnet-Lamb, T. Gee and D. Geraghty, The Sato-Tate conjecture for Hilbert modular forms, http://www.math.harvard.edu/ geraghty/.

[BH] F. Beukers and G. Heckman, Monodromy for the hypergeometric function ${ }_{n} F_{n-1}$, Invent. Math. 95 (1989), 325-354. Zbl 0663.30044 MR 0974906

[C] G. Chenevier, Une application des variétés de Hecke des groupes unitaires, http://www.math.polytechnique.fr/ ${ }^{\sim}$ chenevier/pub.html

[CH] G. Chenevier and M. Harris, Construction of automorphic Galois representations II, http://fa.institut.math.jussieu.fr/node/45

[CHLN] L. Clozel, M. Harris, J.-P. Labesse and B.-C. Ngo (eds.), Stabilisation de la formule des traces, variétés de Shimura, et applications arithmétiques, http:// fa.institut.math.jussieu.fr/node/29

[CHT] L. Clozel, M. Harris and R. Taylor, Automorphy for some $\ell$-adic lifts of automorphic $\bmod \ell$ Galois representations, Publ. Math. IHES 108 (2008), 1-181. Zbl 1169.11020 MR 2470687

[DMOS] P. Deligne, J. S. Milne, A. Ogus and K.-Y. Shih, Hodge cycles, motives and Shimura varieties, Lecture Notes in Math. 900, Springer, 1982. Zbl 0465.00010 MR 0654325 
[Gee] T. Gee, The Sato-Tate conjecture for modular forms of weight 3, Doc. Math. 14 (2009), 771-800. Zbl pre05663795 MR 2578803

[Ger] D. Geraghty, Modularity lifting theorems for ordinary Galois representations, http://www.math.harvard.edu/ geraghty/.

[Gl] J. Glaisher, On the numbers of representations of a number as a sum of $2 r$ squares, where $2 r$ does not exceed eighteen, Proc. London Math. Soc. 5 (1907), 479-490. JFM 38.0225 .03

[GPR] B. Green, F. Pop and P. Roquette, On Rumely's local-global principle, Jahresber. Deutsch. Math.-Verein. 97 (1995), 43-74. Zbl 0857.11033 MR 1341772

[GHK] R. Guralnick, M. Harris and N. Katz, Automorphic realizations of residual Galois representations, J. Eur. Math. Soc. 12 (2010), 915-937. Zbl pre05736056 MR 2654084

$[\mathrm{Gu}] \quad$ L. Guerberoff, Modularity lifting theorems for Galois representations of unitary type, http://www.math.jussieu.fr/ lguerb

[H] M. Harris, Potential automorphy of odd-dimensional symmetric powers of elliptic curves, and applications, in Algebra, arithmetic, and geometry, Progr. Math. 270, Birkhäuser, 2009, 1-21. Zbl pre05778250 MR 2641185

[HSBT] M. Harris, N. Shepherd-Barron and R. Taylor, A family of Calabi-Yau varieties and potential automorphy, Ann. of Math. 171 (2010), 779-813. Zbl pre05712744 MR 2630056

[HT] M. Harris and R. Taylor, The geometry and cohomology of some simple Shimura varieties, Ann. of Math. Stud. 151, Princeton Univ. Press, 2001. Zbl 1036.11027 MR 1876802

[Ka1] N. Katz, Exponential sums and differential equations, Annals of Mathematics Stud. 124, Princeton Univ. Press, 1990. Zbl 0731.14008 MR 1081536

[Ka2] Another look at the Dwork family, in Algebra, arithmetic and geometry. In honour of Y. I. Manin, Vol. II, Progr. Math. 270, Birkhäuser, 2009, 89-126. Zbl 1195.14015 MR 2641188

[Ki] M. Kisin, Moduli of finite flat group schemes, and modularity, Ann. of Math. 170 (2009), 1085-1180. Zbl pre05710184 MR 2600871

[La] J.-P. Labesse, Changement de base CM et séries discrètes, http://people.math. jussieu.fr/ ${ }^{\sim}$ harris/bookproject.html

[LN] G. Laumon and B.-C. Ngo, Le lemme fondamental pour les groupes unitaires, Ann. of Math. 168 (2008), 477-573. Zbl 1179.22019 MR 2434884

[Le] A. Levelt, Hypergeometric functions, thesis, Univ. Amsterdam, 1961. Zbl 0103.29502 MR 0145108

[M] H. Matsumura, Commutative algebra, 2nd ed., Benjamin, 1980. Zbl 0441.13001 MR 0575344

[MB] L. Moret-Bailly, Groupes de Picard et problèmes de Skolem II, Ann. Sci. École Norm. Sup. 22 (1989), 181-194. Zbl 0704.14015 MR 1005158

[R] K. Ribet, On $l$-adic representations attached to modular forms II, Glasgow Math. J. 27 (1985), 185-194. Zbl 0596.10027 MR 0819838

[Se1] J.-P. Serre, Abelian $\ell$-adic representations and elliptic curves, Benjamin, 1968. Zbl 0186.25701 MR 0263823

[Se2] Propriétés galoisiennes des points d'ordre fini des courbes elliptiques, Invent. Math. 15 (1972), 259-331. Zbl 0235.14012 MR 0387283

[Sh] S.-W. Shin, Galois representations arising from some compact Shimura varieties, http://www.math.uchicago.edu/〜 swshin/ 
[SGA7] P. Deligne and N. Katz, Séminaire de géométrie algébrique du Bois-Marie 1967-1969. Groupes de monodromie en géométrie algébrique (SGA 7 II), Lecture Notes in Math. 340, Springer, 1973. Zbl 0258.00005 MR 0354657

[SZ] V. Serežkin and A. Zalesskiı̌, Linear groups generated by transvections, Math. USSRIzv. 10 (1976), 25-46. Zbl 0355.20044 MR 0412295

[Tad] M. Tadić, $G L(n, \mathbb{C})^{\wedge}$ and $G L(n, \mathbb{R})^{\wedge}$, in Automorphic forms and L-functions II: Local aspects, D. Ginzburg et al. (eds.), Amer. Math. Soc. and Bar-Ilan Univ., 2009, 285-313. Zbl 1186.22021 MR 2537046

[Tay] R. Taylor, Automorphy of some $l$-adic lifts of automorphic mod $l$ representations II, Publ. Math. IHES 108 (2008), 183-239. Zbl 1169.11021 MR 2470688

[W] J.-L. Waldspurger, Endoscopie et changement de caractéristique, J. Inst. Math. Jussieu 5 (2006), 423-525. Zbl 1102.22010 MR 2241929 